\title{
Reducing the losses of optical metamterials
}

\author{
by \\ Anan Fang \\ A dissertation submitted to the graduate faculty \\ in partial fulfillment of the requirements for the degree of \\ DOCTOR OF PHILOSOPHY \\ Major: Condensed Matter Physics \\ Program of Study Committee: \\ Costas M. Soukoulis, Major Professor \\ David A. Carter-Lewis \\ Kai-Ming Ho \\ Joseph Shinar \\ Jiming Song
}

Iowa State University

Ames, Iowa

2010

Copyright (c) Anan Fang, 2010. All rights reserved. 


\section{TABLE OF CONTENTS}

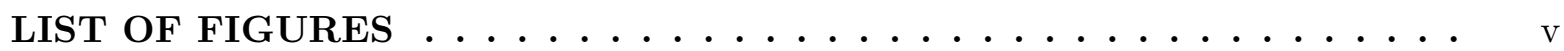

ACKNOWLEDGMENTS ..................... . . . . . . . . .

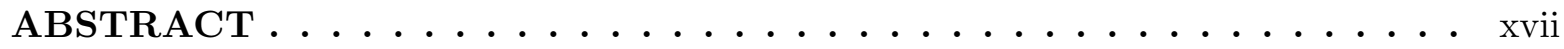

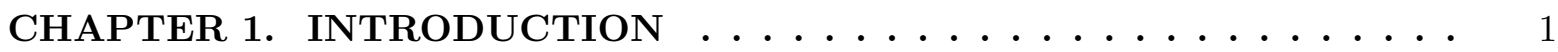

1.1 Electromagnetic Metamaterials . . . . . . . . . . . . . . . . . . 1

1.2 Some Typical Potential Applications of Metamaterials and Their Limitations . 6

1.2.1 Subwavelength imaging . . . . . . . . . . . . . . 6

1.2 .2 Cloaking devices . . . . . . . . . . . . . 7

1.2 .3 Optical nonlinearity . . . . . . . . . . . . . . 8

1.2.4 Zero index of refraction metamaterials . . . . . . . . . 8

1.2.5 The limitations on optical metamaterials f . . . . . . . . . . 9

1.3 The Methods to Reduce the Losses of Optical Metamaterials . . . . . . . . . . 10

1.4 Overview and Layout . . . . . . . . . . . . . . . . . . 13

CHAPTER 2. THE FINITE DIFFERENCE TIME DOMAIN METHOD FOR ELECTROMAGNETICS . . . . . . . . . . . . . 15

2.1 Introduction . . . . . . . . . . . . . . . . . . . 15

2.2 Material Modeling . . . . . . . . . . . . . . . . 16

2.2 .1 Lossy dielectrics . . . . . . . . . . . . . . . . . 16

2.2 .2 Lorentz media . . . . . . . . . . . . . . . . . . 17

2.2 .3 Nonlinear active materials . . . . . . . . . . . . . . . . 20

2.2.4 Perfectly matched layer (PML) absorbing boundary conditions . . . . . 24 
2.3 The Parallel Computation Scheme . . . . . . . . . . . . . . . . . . 28

2.4 Error Analysis at Material Interfaces . . . . . . . . . . . . . . . . . . 31

2.4 .1 TM case . . . . . . . . . . . . . . . . . . . 32

2.4 .2 TE case . . . . . . . . . . . . . . . . . . . . 35

2.5 The Total-field/Scattered-field Technique . . . . . . . . . . . . . . 37

2.5 .1 Ideas . . . . . . . . . . . . . . . . . . . . . 37

2.5.2 Two-dimensional formulation . . . . . . . . . . . . . . . 38

2.5.3 Calculation of the incident field . . . . . . . . . . . . . . . . 43

CHAPTER 3. OPTICAL ANISOTROPIC METAMATERIALS: NEGATIVE REFRACTION AND FOCUSING . . . . . . . . . . . 48

3.1 Introduction $\ldots \ldots \ldots \ldots \ldots \ldots \ldots$

3.2 Superlattice of Metallic-air Layers _ . . . . . . . . . . . . . . . . 50

3.3 Obtained Numerical Dispersion Relations . . . . . . . . . . . . . . . . . 52

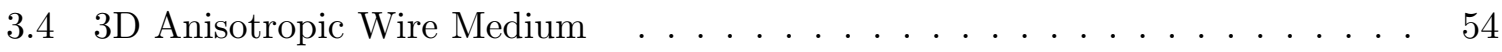

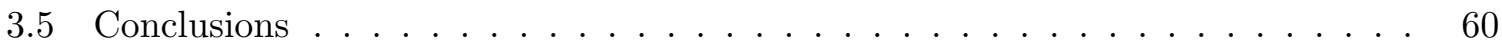

CHAPTER 4. SELF-CONSISTENT CALCULATIONS OF METAMATE-

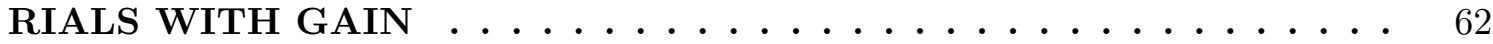

4.1 Introduction . . . . . . . . . . . . . . . . . . . 62

4.2 Lasing and Nonlinear Behaviors in Gain Materials and Metamaterials Incorporated with Gain . . . . . . . . . . . . . . . . . . . 65

4.2 .1 Gain material only . . . . . . . . . . . . . . . . 65

4.2.2 Negative index material (NIM) embedded in layers of gain . . . . . . . . 69

4.2 .3 Conclusions . . . . . . . . . . . . . . . . . . . 75

4.3 Loss Compensation in 2D Metamaterials . . . . . . . . . . . . . . 76

4.3.1 One layer of gain material embedded in a square lattice of Lorentz dielectric cylinders . . . . . . . . . . . . . . . 76

4.3.2 2D split ring resonators (SRRs) with gain material inclusions . . . . . 80

4.3 .3 Conclusions . . . . . . . . . . . . . . . . . . . . 83 
4.4 Overcoming Losses with Gain in Fishnet Metamaterials . . . . . . . . . . . . . 84

4.4.1 Geometric dimensions of the fishnet structure . . . . . . . . . . . . . 84

4.4.2 Transmission $T$, reflection $R$, and absorption $A$ spectra and retrieval results .......................... 85

4.4.3 Kramers-Kronig relations for metamaterials with gain . . . . . . . . 86

4.4 .4 Conclusions . . . . . . . . . . . . . . . . . 87

4.5 Overcoming Losses with Gain in 3D SRR Metamaterials . . . . . . . . . . 88

4.5.1 Geometric dimensions of 3D SRR metamaterials . . . . . . . . . . . 88

4.5.2 Numerical simulations and discussions . . . . . . . . . . . . . . . . . 89

4.5 .3 Conclusions . . . . . . . . . . . . . . . . . . . . 98

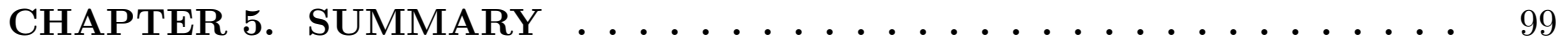

APPENDIX A. THE 3D FDTD UPDATE ALGORITHM . . . . . . . . . 102

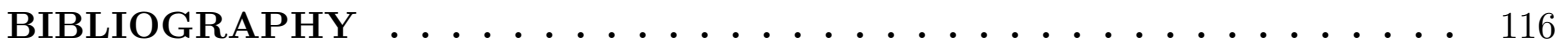




\section{LIST OF FIGURES}

Figure 1.1 Illustration of a negative refraction at the interface between a positiveindex material (PIM) and a negative-index material (NIM). Note that the phase velocity is in the direction opposite to that of energy flow in NIM. . . . . . . . . . . . . . . . . .

Figure 1.2 (a) The two concentric split-ring resonator, taken from Ref. [14]. (b) The first experimentally realized negative index material in microwave regime (see Ref. [14]). . . . . . . . . . . . . . . .

Figure 1.3 Illustration of Snell's law experiments on a NIM wedge. Note, the exiting beam is bent to different sides of the surface normal for positive and negative refractions. . . . . . . . . . . . .

Figure 1.4 Illustration of perfect lensing, taken from Ref. [4]. Both the propagating waves (A) and the evanescent waves (B) are restored in the image. .

Figure 1.5 (a) Illustration of an array of short-wire pairs, taken from Ref. [27].

(b) Top-view electron micrograph of the silver-based fishnet structure, taken from Ref. $[29] . \ldots \ldots \ldots \ldots \ldots$

Figure 1.6 Illustration of the cloaking mechanism using metamaterials. (A) A 2D cross-section of rays trajectories in the cloaking system. (B) A 3D view. Figures are taken from Ref. [3]. The cloaked object (orange) is coated by a layer of metamaterials (blue), for which $\varepsilon$ and $\mu$ are spatially modulated, based on coordinate transformations $[3] . \ldots \ldots$ 
Figure 1.7 (a) Schematic of ray refractions at the material interface. (b) Rays emanating from a point source inside zero index metamaterials exit from the slab as a plane wave. . . . . . . . . . . . . 8

Figure $2.1 \quad$ Schematic of the leap-frog scheme. . . . . . . . . . . . . . . 16

Figure $2.2 \quad$ Schematic of the four-level atomic system model. . . . . . . . . . . . . 21

Figure $2.3 \quad$ Schematic of the parallel computation scheme. . . . . . . . . . . . 28

Figure 2.4 Illustration of the extension of each computation domain by a foreign edge buffer to communicate the edge field data. . . . . . . . . . . . 29

Figure $2.5 \quad$ Improper message passing order leading to deadlock. . . . . . . . . . . 29

Figure 2.6 The communication scheme between neighboring nodes. . . . . . . . 30

Figure 2.7 Illustration of Yee grids in the TM case. In the light blue and green area, the dielectric constants are $\varepsilon_{1}$ and $\varepsilon_{2}$, respectively. Both of them have the permeability $\mu=\mu_{0}$. The red line at the position $x=x_{\text {mat }}$ is the material interface. The Yell cell size is $h \times h . \ldots . \ldots 32$

Figure 2.8 Illustration of Yee grids in the TE case. All others are the same as Figure $2.7 . \ldots \ldots \ldots \ldots \ldots \ldots \ldots \ldots$

Figure 2.9 Illustration of the TF/SF zoning in the computation space domain. $\quad 38$

Figure 2.10 The Yee grid for the 2D TE case. The solid line indicates the interface between Region 1 (inside) and Region 2 (outside), which is aligned with $E_{z}$ components. The incident field data are required for $H_{x}$ and $H_{y}$ in the Yee cells surrounding the interface, and $E_{z}$ at the interface. . . . .

Figure 2.11 The Yee grid for the 2D TM case. The solid line indicates the interface between Region 1 (inside) and Region 2 (outside), which is aligned with the magnetic field components. The incident field data are required for $H_{z}$ at the interface, and $E_{x}$ and $E_{y}$ in the Yee cells surrounding the

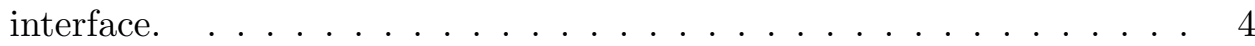


Figure 2.12 Illustration of the incident wave impinged on Region 1 from four different directions. In each direction, Region 1 has a different first contacted grid point by the incident wavefront. . . . . . . . . . . . . . . . 44

Figure $3.1 \quad$ (a) Top graph: Circular equifrequency surfaces (EFS) for vacuum and isotropic media. Bottom graph: Equifrequency surfaces for vacuum (circle) and negative anisotropic refraction media (hyperbolic relation). (b) The definitions for $k_{\perp}, k_{\|}, \varepsilon_{\perp}$, and $\varepsilon_{\|}$used in our simulations. .

Figure 3.2 Material parameters of the metallic layers: $\varepsilon=-4, \mu=1$; working frequency $f=0.5 \mathrm{GHz}$; space period of metallic layers is $0.06 \mathrm{~m}$; width of metallic layers is $0.02 \mathrm{~m}$. (a) The magnetic field distribution of the group negative refraction in the metallic-air layers array slab for a transverse magnetic Gaussian beam with an incident angle of $30^{\circ}$. The white line indicates the ray-tracing result. (b) A ray-tracing diagram showing that the rays coming from a line source are refocused by an anisotropic medium slab with the effective permittivity $\varepsilon$ tensor of our simulated metallic-air layers array slab. (c) The magnetic field distribution of the pseudo focusing of our simulated metallic-plates array slab with a line source placed $1.25 \mathrm{~m}$ from the interface, which launches a cylindrical transverse magnetic polarized wave. The thickness and the width of the metallic-air layers array slab are $2.4 \mathrm{~m}$ and $6 \mathrm{~m}$, respectively. (d) The magnetic field distribution of the pseudo focusing in a homogeneous anisotropic slab with the effective permittivity of our simulated metallic-air layers array slab $\left(\varepsilon_{\|}=1.7293, \varepsilon_{\perp}=-0.7907\right) . \ldots 51$ 
Figure 3.3 (a) Schematic of 3D metallic wires embedded in a dielectric matrix. (b) The magnetic field distribution for the negative refraction in a 3D goldwire square-lattice medium with vacuum background and the wavelength $\lambda=700 \mathrm{~nm}$. The incident plane wave has transverse magnetic polarization and an incident angle of $45^{\circ}$. The permittivity $\varepsilon$ for gold is taken from experimental data [116]: $\varepsilon=-15.5931+i 1.2734$ at $\lambda=700 \mathrm{~nm}$. The radius, the length of gold wires, and the lattice constant are 16, 1532 and $70 \mathrm{~nm}$, respectively. The white arrow indicates the direction of power flow. . . . . . . . . . . . . 53

Figure 3.4 The magnetic field distribution of the pseudo focusing in a 3D gold-wire square-lattice medium with a line source placed $884 \mathrm{~nm}$ away from the interface, which launches a cylindrical transverse magnetic polarized wave at the wavelength $\lambda=700 \mathrm{~nm}$. The permittivity of gold is the same as in Figure 3.3. The background is vacuum. The radius, length of gold wires, and the lattice constant are 16, 2732 and $70 \mathrm{~nm}$, respectively. The white arrow indicates the direction of power flow. . . . . . . . 54

Figure 3.5 The numerical dispersion relation data from the simulation (solid circles) and the fitted hyperbolic curve (dashed line). All parameters are the same as in Figure 3.4, except the length of gold wires which is $1500 \mathrm{~nm}$. Note that all $k$ components here are normalized by $k_{0}$, where $k_{0}=\omega / c$. The inset shows, as a typical example, the field distribution for $\theta_{i}=30^{0}$ fitted by Eq. 3.2. . . . . . . . . . . . . . . 55

Figure 3.6 The effective permittivity $\varepsilon_{\perp}$ and $\varepsilon_{\|}$calculated from Maxwell-Garnett equations (solid lines) and numerical simulations (squares) for different wire radii. The simulated medium is a $3 \mathrm{D}$ square lattice silver wire medium in vacuum with the lattice constant $a=20 \mathrm{~nm}$. The wavelength is $\lambda=700 \mathrm{~nm}$. The permittivity of silver at $\lambda=700 \mathrm{~nm}$ is $\varepsilon_{\text {silver }}=-20.4373+i 1.2863$, taken from experimental data [116] . . . 56 
Figure 3.7 (a) The magnetic field distribution of the focusing simulation for the simulated 3D gold-wire square lattice anisotropic medium slab, with the source $884 \mathrm{~nm}$ away from the first interface. (b) Same as (a), but for a homogeneous anisotropic slab with the fitted effective parameters $\varepsilon_{\|}=1.4455+i 0.0044$ and $\varepsilon_{\perp}=-1.9082+i 0.2391$. (c) and (d) are the same as (a) and (b), respectively, but for the magnetic field intensity distribution. (e) - (h) are the same as $(\mathrm{a})-(\mathrm{d})$, respectively, except the source is $442 \mathrm{~nm}$ away from the first interface. All material parameters are the same as in Figure 3.4. . . . . . . . . . . . . . . 57

Figure 3.8 The magnetic field distribution in a 3D silver-wire hexagonal lattice medium slab with the alumina background. The incident plane wave has the transverse magnetic polarization and the wavelength in vacuum $\lambda=700 \mathrm{~nm}$. (a) Normal incidence. (b) At an incident angle of $30^{0}$. The white arrow indicates the direction of power flow. The hexagonal lattice constant $a$, the radius $r$, and the length $l$ of silver wires are $120 \mathrm{~nm}, 30 \mathrm{~nm}$ and $1700 \mathrm{~nm}$, respectively. The permittivities of silver and alumina at the wavelength in vacuum $\lambda=700 \mathrm{~nm}$ are $\varepsilon_{\text {silver }}=-20.4373+i 1.2863$ and $\varepsilon_{\mathrm{Al}_{2} \mathrm{O}_{3}}=3.1$, respectively, taken from experimental data $[116] . \ldots \ldots \ldots \ldots$. . . . . . . . . . . . . . 58

Figure 3.9 The numerical dispersion (solid circles) and the fitted dispersion curve (dashed line) of 3D silver-wire hexagonal-lattice media in the alumina background. (a) The lattice constant $a=120 \mathrm{~nm}$ and the radius of silver wires $r=30 \mathrm{~nm}$. (b) The lattice constant $a=30 \mathrm{~nm}$ and the radius of silver wires $r=12 \mathrm{~nm} . \quad k_{\|} \leq k_{0}$ corresponds to the propagating modes in the background, while $k_{\|}>k_{0}$ corresponds to the evanescent modes. All other parameters are the same as in Figure 3.8. Note that all $k$ components are normalized by $k_{0}$, where $k_{0}=\sqrt{\varepsilon} \omega / c$ and $\varepsilon$ is the permittivity of alumina. . . . . . . . . . . . 59 
Figure 4.1 Schematic of gain material slab (shown in orange). The slab width $w$ takes different values in the cases we have examined. . . . . . . . . 65

Figure 4.2 The transmitted waves and their corresponding Fourier transforms for different input powers. (a), (c) and (e) are the transmitted waves for input power $P_{\text {in }}=79.6,90.7$ and $120.6 \mathrm{~W} / \mathrm{mm}^{2}$, respectively. (b), (d) and (f) are same as (a), (c) and (e), respectively, but for the Fourier transforms of the transmitted waves. The gain slab width $w=100 \mathrm{~nm}$ and the bandwidth $\Gamma_{a}$ of the atomic transition between $N_{1}$ and $N_{2}$ is

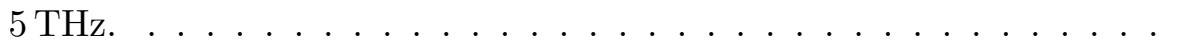

Figure 4.3 The powers emitted at the emission frequency $\omega=\omega_{a}(100 \mathrm{THz})$ for different input powers at the pumping frequency $\omega=\omega_{b}(200 \mathrm{THz})$. All parameters of this system are same as Figure $4.2 \ldots \ldots$. . . . . . 67

Figure 4.4 The lasing times for different input powers at the pumping frequency $\omega_{b}$ (200 THz). The gain slab width $w=100,250$ and $500 \mathrm{~nm}$, respectively.

All other parameters are same as Figure 4.2. . . . . . . . . . . . 68

Figure 4.5 The amplitude of the input EM wave inside the gain slab as a function of the position. The gain slab width $w=1000 \mathrm{~nm}$ and the input power $P_{\text {in }}=92.3 \mathrm{~W} / \mathrm{mm}^{2}$. All other parameters are same as Figure 4.2 . . .

Figure 4.6 The powers emitted at the emission frequency $\omega=\omega_{a}(100 \mathrm{THz})$ for different pumping rates. The gain slab width $w=100 \mathrm{~nm}$ and the bandwidth of the atomic transition between $N_{1}$ and $N_{2}$ is $5 \mathrm{THz} . \quad$. . 70

Figure 4.7 The normalized occupation numbers as a function of time. The gain slab width $w=100 \mathrm{~nm}$ and the gain bandwidth of the atomic transition between $N_{1}$ and $N_{2}$ is $5 \mathrm{THz}$. (a) The electrons are optically pumped by an input EM wave with input power $P_{\text {in }}=120.6 \mathrm{~W} / \mathrm{mm}^{2}$ and (b) the electrons are pumped with a homogeneous pumping rate $\Gamma_{\text {pump }}=$ $9.3 \times 10^{9} \mathrm{~s}^{-1}$. Occupation numbers $N_{0}, N_{1}, N_{2}$ and $N_{3}$ are normalized by the total electron density $N_{i}\left[N_{i}=N_{0}(t=0)=5.0 \times 10^{23} / \mathrm{m}^{3}\right] . \quad$. 71 
Figure 4.8 The negative index material (blue) embedded in layers of gain material (orange). The number of layers, the permittivity and permeability of NIM are taken different values for different cases we have examined. The width for both NIM and gain material is $w=50 \mathrm{~nm}$. The gain bandwidth is $5 \mathrm{THz} . \ldots \ldots \ldots \ldots \ldots$

Figure 4.9 The transmission vs. signal amplitude for the loss-compensated metamaterial of a three-layer system (NIM - gain material - NIM) with gain bandwidth of $5 \mathrm{THz}$, for different pumping rates $\Gamma_{\text {pump }} . \Gamma_{\text {pump }}$ is increased from $4.0 \times 10^{9} \mathrm{~s}^{-1}$ (lowest) to $5.5 \times 10^{9} \mathrm{~s}^{-1}$ (highest) in steps of $1.0 \times 10^{8} \mathrm{~s}^{-1}$. The material parameters for NIM are $\varepsilon=\mu=-1+2 i$. The metamaterial response is linear in a very wide range. When the loss-compensated transmission reaches exactly unity, the pumping rate $\Gamma_{\text {pump }}=4.70 \times 10^{9} \mathrm{~s}^{-1}$, which is called the critical pumping rate. For incident fields stronger than $10^{4} \mathrm{~V} / \mathrm{m}$ the metamaterial behaves nonlin-

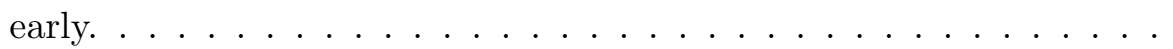

Figure 4.10 The transmission vs. signal amplitude for the loss-compensated metamaterial of Figure 4.8 with gain bandwidth of $5 \mathrm{THz}$ at the critical pumping rates $\Gamma_{\text {pump }}=1.98 \times 10^{9} \mathrm{~s}^{-1}$. The material parameters for NIM are same as Figure 4.9. For incident fields stronger than $10^{3} \mathrm{~V} / \mathrm{m}$ this metamaterial becomes non-linear. . . . . . . . . . . . . . .

Figure 4.11 The critical pumping rates for different imaginary parts of the refractive index $n$ of NIMs. The structure is a three-layer system (NIM - gain material - NIM) and $\varepsilon=\mu$ for NIMs. . . . . . . . . . . . . . . 74

Figure 4.12 The numerical and analytical results for the susceptibilities of gain materials as a function of frequency. (a) a three-layer system (NIM - gain material -NIM) at the critical pumping rate $\Gamma_{\text {pump }}=4.7 \times 10^{9} \mathrm{~s}^{-1}$. (b) a 19-layer system of Figure 4.8 at the critical pumping rate $\Gamma_{\text {pump }}=$

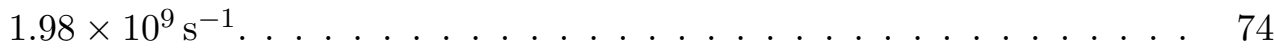


Figure 4.13 (a) The time-dependent electric field of the transmitted wave for a threelayer system (NIM - gain material - NIM) and (b) the corresponding Fourier transform in frequency domain for the lasing in (a). The pumping rate $\Gamma_{\text {pump }}=1.5 \times 10^{10} \mathrm{~s}^{-1} \ldots \ldots \ldots \ldots 75$

Figure 4.14 One layer of gain material (orange) embedded in a square lattice of dielectric square cylinders (blue) that have a Lorentz behavior. The dielectric constant of the cylinders is given by $\varepsilon=1+\omega_{p}^{2} /\left(\omega_{p}^{2}-2 i \omega \gamma-\right.$ $\left.\omega^{2}\right)$, where the resonance frequency $f_{p}=\omega_{p} / 2 \pi=100 \mathrm{THz}$ and $\gamma=$ $2 \pi f$, and $f$ takes different values in the cases we have examined. The dimensions are $a=80 \mathrm{~nm}, w_{L}=40 \mathrm{~nm}$, and $w_{g}=30 \mathrm{~nm} . \ldots$.

Figure 4.15 (a) The retrieved results for the real and the imaginary parts of the effective permittivity $\varepsilon$ with gain and without gain. Below compensation, $t=0.89$; gain and Lorentz bandwidths are $20 \mathrm{THz}$ and $5 \mathrm{THz}$, respectively. (c) and (e) are same as (a), but for the loss compensated case $(t=1)$ and the overcompensated case $(t=1.34)$, respectively. (b), (d) and (f) are same as (a), (c) and (e), respectively, except with gain

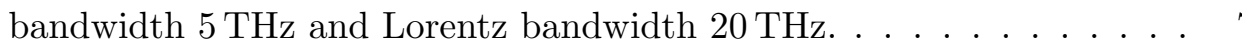

Figure 4.16 Geometry for a unit cell of the square SRR system with gain embedded in the gap (orange). The dimensions are $a=100 \mathrm{~nm}, l=80 \mathrm{~nm}, t=$ $5 \mathrm{~nm}, d=5 \mathrm{~nm}$ and $w=15 \mathrm{~nm} \ldots \ldots \ldots \ldots$

Figure 4.17 The retrieved results for the real and the imaginary parts of (a) the effective permeability $\mu$ and (b) the corresponding effective index of refraction $n$, with and without gain for a pumping rate $\Gamma_{\text {pump }}=1.0 \times$ $10^{9} \mathrm{~s}^{-1}$ and the SRR system of Figure 4.16. The gain bandwidth is $20 \mathrm{THz}$. Notice that the width of the magnetic resonance with gain is $2.61 \mathrm{THz} \ldots \ldots \ldots \ldots \ldots \ldots \ldots \ldots$ 
Figure 4.18 The retrieved results for the real and the imaginary parts of the effective permeability $\mu$ with and without gain for a pumping rate $\Gamma_{\text {pump }}=$ $8.0 \times 10^{8} \mathrm{~s}^{-1}$ and the SRR system where the SRR is surrounded by gain. The gain bandwidth is $20 \mathrm{THz}$. Notice that the width of the magnetic resonance with gain is $1.60 \mathrm{THz}$, narrower than Figure 4.17(a). 82

Figure 4.19 Schematic of the unit cell of the fishnet structure with the parameters marked on it. The geometric parameters are $a_{x}=a_{y}=860 \mathrm{~nm}, a_{z}=$ $200 \mathrm{~nm}, w_{x}=565 \mathrm{~nm}, w_{y}=265 \mathrm{~nm}, s=50 \mathrm{~nm}, t=30 \mathrm{~nm}$ and $h_{g}=$ $20 \mathrm{~nm}$. The thicknesses of the metal (silver) and gain layer are $t$ and $h_{g}$, respectively, and the dielectric constant of the spacer, $\mathrm{MgF}_{2}$, is 1.9. These parameters were used on simulations [Ref. [128]] and experiments [Ref. [28]]. . . . . . . . . . . . . . . . . . . . 84

Figure 4.20 The (a) transmission, (b) reflection, and (c) absorption as a function of wavelength for different pumping rates. . . . . . . . . . . . . 85

Figure 4.21 The retrieved results for the real (solid lines) and the imaginary (dashed lines) parts of (a) the effective permeability, $\mu$, and (b) the corresponding effective index of refraction, $n$, without and with gain for different pumping rates. (c) The figure-of-merit (FOM) as a function of wavelength for different pumping rates. The gain bandwidth is $20 \mathrm{THz} . \quad$. . 87

Figure 4.22 The real and imaginary parts of the retrieved effective permeability, $\mu$, and the results from the Kramers-Kronig relations for pumping rates (a) $\Gamma_{\text {pump }}=5.0 \times 10^{8} \mathrm{~s}^{-1}$ (below compensation) and (b) $\Gamma_{\text {pump }}=6.9 \times$ $10^{8} \mathrm{~s}^{-1}$ (overcompensated). . . . . . . . . . . . . . 88 
Figure 4.23 (a) One unit cell for the silver-based SRR structure (light blue) with the gain layer underneath. The dielectric constants $\varepsilon$ for GaAs (yellow) and gain (red) are 11 and 2, respectively. The whole structure is in vacuum background (light gray). The dimensions are $a=250 \mathrm{~nm}, l=160 \mathrm{~nm}$, $h=80 \mathrm{~nm}, h_{1}=15 \mathrm{~nm}, h_{2}=h_{3}=10 \mathrm{~nm}, h_{4}=45 \mathrm{~nm}, h_{s}=25 \mathrm{~nm}$, $w=40 \mathrm{~nm}$ and $d=20 \mathrm{~nm}$. (b) same as (a) except the gain is embedded in the SRR gap with $\varepsilon=1$ and the gain layer in (a) is replaced by a dielectric layer $(\varepsilon=2)$ (blue) . . . . . . . . . . . . . .

Figure 4.24 The retrieved results for the real and imaginary parts of the effective permeability $\mu$, with and without gain, for the gain configuration shown in Figure $4.23(\mathrm{a})$. For the case with gain, the pumping rate $\Gamma_{\text {pump }}=$ $1.5 \times 10^{9} \mathrm{~s}^{-1} \ldots \ldots \ldots \ldots \ldots \ldots \ldots$

Figure 4.25 The real (solid) and imaginary (dashed) parts of $I /\left(\eta \omega^{2} E\right)$ as a function of frequency for different pumping rates. (a) For the structure with a gain layer below the SRR, shown in Figure 4.23(a). (b) For the structure with the gain in the SRR gap as shown in Figure 4.23(b). Notice that the resonance is getting stronger and narrower as the pumping rate increases. . . . . . . . . . . . . . . .

Figure 4.26 The retrieved results for the real and imaginary parts of the effective permittivity $\varepsilon$, with and without gain, for the normal incidence in Figure 4.23(a). For the case with gain, the pumping rate $\Gamma_{\text {pump }}=$ $1.0 \times 10^{9} \mathrm{~s}^{-1} \ldots \ldots \ldots \ldots \ldots \ldots \ldots$

Figure 4.27 Top view of the gain layer in Figure 4.23(a) when a shadow (blue) is cast by the SRR structure. The gain goes away in the shadow, while in other area $($ red) it is homogeneously pumped. . . . . . . . . . . 
Figure 4.28 The real (solid) and imaginary (dashed) parts of $I /\left(\eta \omega^{2} E\right)$ as a function of frequency for different pumping rates. (a) For the structure with a shadow in the gain layer cast by the SRR. (b) For the structure where the gain is pumped in $y$ direction only (see the coordinate system in Figure $4.23(\mathrm{a})) . \ldots \ldots \ldots \ldots$. . . . . . . . . . . . . . . . .

Figure 4.29 The electric field amplitude distribution at the resonance frequency in the cross-section of the gain layer ( $x y$ plane in Figure 4.23(a)) for different components: (a) $E_{x}$, (b) $E_{y}$ and (c) $E_{z}$. The area enclosed by the black line is the projection of the SRR in the gain layer. The electric field is calculated without gain. . . . . . . . . . .

Figure 4.30 The imaginary parts of $I /\left(\eta \omega^{2} E\right)$ as a function of frequency for different background dielectric constants of the gain material, which is only pumped in $y$ direction. For the case with gain, the pumping rate is $\Gamma_{\text {pump }}=1.5 \times 10^{9} \mathrm{~s}^{-1}$. Note the resonance enhancements by the gain are almost the same. . . . . . . . . . . . . . . . 96

Figure 4.31 The electric field amplitude distributions for different background dielectric constants of the gain material $\left(\varepsilon_{g}=2,5\right.$ and 11) at their corresponding resonance frequencies, in a $y z$ plane crossing the middle of the gap bearing side of the SRR. (a) $E_{y}$ and (b) $E_{z}$. The area enclosed by the dashed black line indicates the position of the gain layer. The electric field is calculated without gain. . . . . . . . . . . . 9 97

Figure A.1 Schematic of the Yee cell used in discretizing the space. . . . . . . . . 102

Figure A.2 Schematic of the 3D FDTD computation space and the polarization of the incident fields. . . . . . . . . . . . . . . . . . 105 


\section{ACKNOWLEDGMENTS}

I am happy to have this opportunity to thank those who helped me in conducting research and enjoying my time at Iowa State University. First and foremost, I would like to express my thanks to my advisor, Dr. Costas M. Soukoulis, for his guidance, patience, and support throughout my graduate study. His deep insights have often inspired me and opened new paths to conduct my research. I learned a lot from him about how to be a true scientist, which will benefit me greatly in my future endeavors.

I would also like to sincerely thank my committee members for serving on my committee and their useful discussions: Drs. David A. Carter-Lewis, Kai-Ming Ho, Joseph Shinar, and Jiming Song. I would additionally like to thank Dr. David A. Carter-Lewis for his continuing to serve on my committee after he retired. I have also benefited a lot from courses that I took from Dr. Jiming Song.

I also want to say thanks to my current and former colleagues at Iowa State University, especially to Dr. Thomas Koschny, for his patient and kind discussions when answering questions and solving problems. He has never disappointed us. His efforts at work also inspired us to work harder. Thanks are also given to Drs. Bingnan Wang, Weitao Dai, Nianhai Shen, Yanhong Zou, Zhixiang Huang, Jiangfeng Zhou, Lei Zhang, Philippe Tassin, Durdu Guney and Marcus Diem, and Rongkuo Zhao for useful discussions and taking time out for fun.

In addition, I would like to take this opportunity to express my thanks to all my friends who have ever helped me in my life and my research. I really enjoyed my times with them.

Finally, I want to thank my family for their encouragement and never-failing support. I really appreciate this. 


\begin{abstract}
The field of metamaterials is driven by fascinating and far-reaching theoretical visions, such as perfect lenses, invisibility cloaking, and enhanced optical nonlinearities. However, losses have become the major obstacle towards real world applications in the optical regime. Reducing the losses of optical metamaterials becomes necessary and extremely important.

In this thesis, two approaches are taken to reduce the losses. One is to construct an indefinite medium. Indefinite media are materials where not all the principal components of the permittivity and permeability tensors have the same sign. They do not need the resonances to achieve negative permittivity, $\varepsilon$. So, the losses can be comparatively small. To obtain indefinite media, three-dimensional (3D) optical metallic nanowire media with different structures are designed. They are numerically demonstrated that they are homogeneous effective indefinite anisotropic media by showing that their dispersion relations are hyperbolic. Negative group refraction and pseudo focusing are observed.

Another approach is to incorporate gain into metamaterial nanostructures. The nonlinearity of gain is included by a generic four-level atomic model. A computational scheme is presented, which allows for a self-consistent treatment of a dispersive metallic photonic metamaterial coupled to a gain material incorporated into the nanostructure using the finitedifference time-domain (FDTD) method. The loss compensations with gain are done for various structures, from 2D simplified models to 3D realistic structures. Results show the losses of optical metamaterials can be effectively compensated by gain. The effective gain coefficient of the combined system can be much larger than the bulk gain counterpart, due to the strong local-field enhancement.
\end{abstract}




\section{CHAPTER 1. INTRODUCTION}

\section{$1.1 \quad$ Electromagnetic Metamaterials}

Electromagnetic metamaterials are artificially constructed structures with extraordinary electromagnetic properties which do not exist in naturally occurring materials [1-12]. More than 40 years ago, it was first proposed by V. G. Veselago [1] that in a medium for which the electric permittivity, $\varepsilon$, and the magnetic permeability, $\mu$, are simultaneously negative, the wave vector, $\mathbf{k}$, electric field, $\mathbf{E}$, and magnetic field, $\mathbf{H}$, can form a left-handed set, based on Maxwell's equations. Hence, it is called a left-handed material (LHM). It follows that the direction of phase velocity is opposite to that of energy flow in LHMs. Hence, the refractive index, $n$, must be negative. The negative refractive index brings an alternative name, negative index material (NIM), and means at an interface between a conventional positive-index material (PIM) and a negative-index material, the refracted electromagnetic (EM) wave is bent to the 'wrong' side with respect to the normal, i.e., it experiences a negative refraction (shown in Figure 1.1). This is an unusual electromagnetic property which does not exist in any naturally occurring materials and provides an opportunity to rethink the interpretation of very basic laws. However, Veselago's early work did not raise much interest from the researchers at that time because no known natural materials exhibit a frequency range with $\varepsilon<0$ and $\mu<0$ simultaneously. Negative permittivity materials are very common. Many metals, such as silver and gold, have negative $\varepsilon$ up to the visible spectrum below their plasma frequencies. However, it is hard to find natural materials with negative permeability, $\mu$, except some ferromagnetic materials for which magnetic resonances typically die out at microwave frequencies. Natural diamagnetic materials have very weak magnetic responses. For example, the susceptibility of the most strongly diamagnetic material, bismuth, is $\chi=-1.66 \times 10^{-4}$. So the permeability, 
$\mu$, can only be slightly less than 1 .

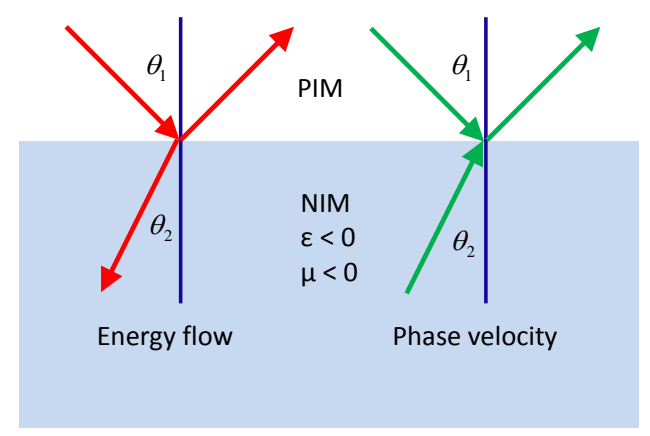

Figure 1.1 Illustration of a negative refraction at the interface between a positive-index material (PIM) and a negative-index material (NIM). Note that the phase velocity is in the direction opposite to that of energy flow in NIM.

The situation changed in 1999, when Pendry [13] suggested that $\mu<0$ can be achieved by two concentric split-ring resonators (SRRs)(Figure 1.2(a)) made of nonmagnetic materials. The idea is the SRR can be treated by a simple LCR model, which can exhibit a magnetic resonance when the incident magnetic field is oriented perpendicular to the SRR plane. If the magnetic resonance is strong enough, the negative permeability, $\mu$, is obtained in a frequency band above the resonance frequency. The magnetic resonance frequency can be modulated by choosing the SRR parameters, such as the size of the ring and gap of the SRR.

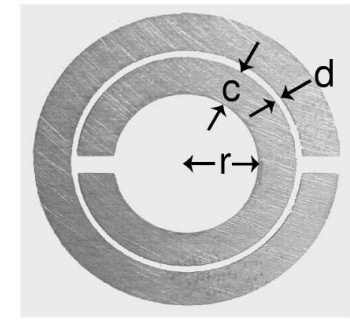

(a)

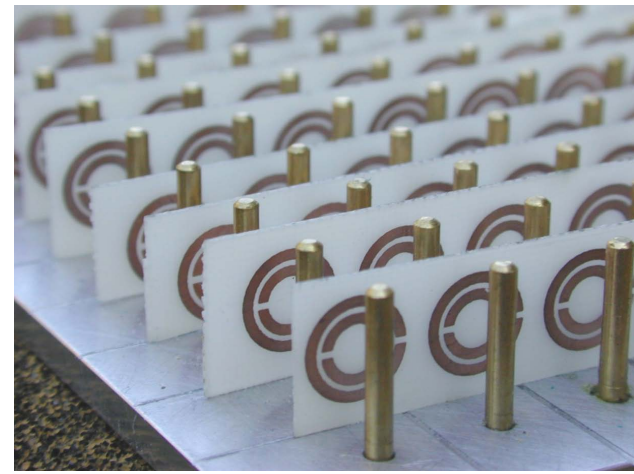

(b)

Figure 1.2 (a) The two concentric split-ring resonator, taken from Ref. [14]. (b) The first experimentally realized negative index material in microwave regime (see Ref. [14]). 
Following Pendry's pioneer work, Smith et al. [14] first demonstrated the negative index material with both $\varepsilon$ and $\mu$ negative in a frequency range in the gigahertz regime in 2000, by interleaving the SRR lattice with a lattice of metallic wires (Figure 1.2(b)). The negative permittivity comes from the lattice of metallic wires, for which the plasma frequency can be significantly lowered because the wire lattice dilutes the average concentration of electrons and considerably enhance the effective electron mass through self-inductance [15]. By choosing the parameters of the lattice of metallic wires, we can achieve the plasma frequency above the resonance frequency of the SRR. Hence, the composite material can have an overlapping region where $\varepsilon<0$ and $\mu<0$. Later, the existence of negative refractive index $n$ was further demonstrated by different groups [16-18] through Snell's law experiments on a wedge-shaped NIM (Figure 1.3).

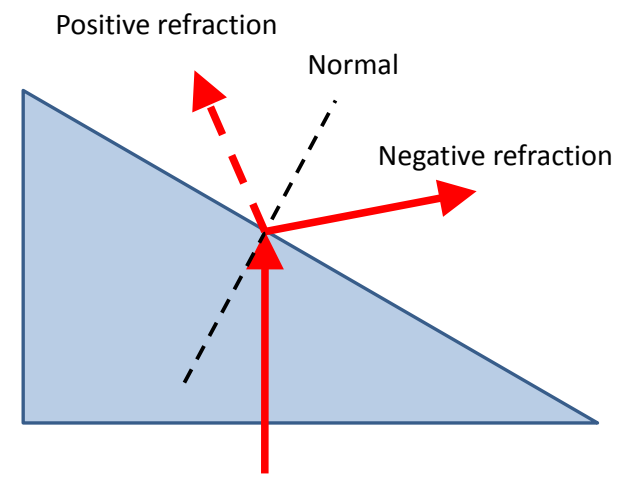

Figure 1.3 Illustration of Snell's law experiments on a NIM wedge. Note, the exiting beam is bent to different sides of the surface normal for positive and negative refractions.

Another milestone development in NIMs is Pendry's famous perfect lens prediction in 2000 [2]. He showed a flat NIM slab, with $\varepsilon=-1$ and $\mu=-1$, can bring together not only the propagating waves but also the evanescent waves carrying finer details of the object (Figure 1.4), such that it can achieve a super-resolution beyond the diffraction limit. The diffraction limit is roughly half a wavelength and constrains all conventional lens.

Inspired by the successful experimental realization of NIMs and Pendry's perfect lens prediction, research on metamaterials has experienced an explosive growth. More and more re- 

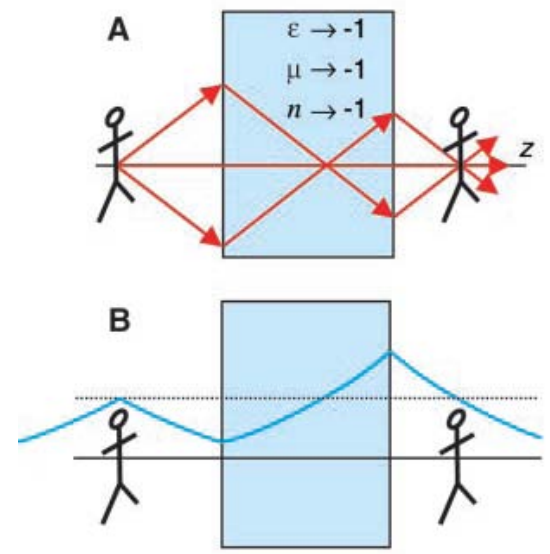

Figure 1.4 Illustration of perfect lensing, taken from Ref. [4]. Both the propagating waves (A) and the evanescent waves (B) are restored in the image.

search has been conducted on various metamaterial structures, and more intriguing electromagnetic properties have been discovered. By scaling down the size of the SRR and geometry optimization, researchers have brought artificial magnetism from the microwave regime to the optical frequency [19-25]. The negative permeability, $\mu$, is achieved up to the red light by Yuan et al. [25] at Purdue University. This is amazing, because optical magnetism does not exist in natural materials. The existence of the negative refractive index also has been pushed into optical wavelengths by introducing new designs [5-7, 26-30] such as short-wire pairs [27] and fishnet structures [26, 28-30] (see Figure 1.5), which enables exciting applications of negative index materials.

Although primary research in metamaterials investigates materials with negative refractive index, metamaterials are not limited to NIMs. They also include artificial dielectrics, artificial magnetic materials, and bi-isotropic and bi-anisotropic composites (such as chiral metamaterials), etc. In a more general case, metamaterials are beyond the field of electromagnetics and the idea has been introduced in the research of acoustics and seismology [31-34]. This is an example where the identification of new material parameters can prompt the development of similar concepts in similar research areas. The word 'meta' means 'beyond' in Greek, and in this sense, metamaterials are defined as materials beyond conventional materials. In conven- 

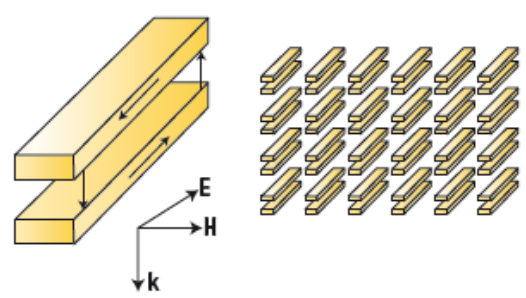

(a)

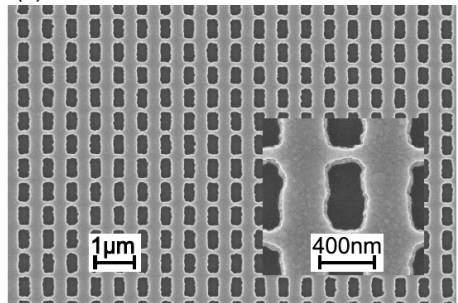

(b)

Figure 1.5 (a) Illustration of an array of short-wire pairs, taken from Ref. [27]. (b) Top-view electron micrograph of the silver-based fishnet structure, taken from Ref. [29].

tional materials, light does not 'see' the details of atoms because the illumination wavelength is hundreds of times larger than the atoms, so the materials can be considered as a homogeneous medium characterized by their macroscopic material parameters, such as the electric permittivity, $\varepsilon$, and the magnetic permeability, $\mu$, based on the averaged responses over the atomic scale. The key idea of metamaterials is to mimick this electromagnetic phenomena of conventional materials, and replace the atoms and molecules of a conventional material with deep subwavelength predesigned artificial inclusions, i.e., meta-atoms, to achieve a homogeneous medium with unprecedented macroscopic electromagnetic properties and functionalities. In general, metamaterials have the following features:

- Artificially constructed periodic structures;

- Exhibit exceptional properties not readily found in natural materials, which result from the design of artificial inclusions;

- The sizes of artificial inclusions and the structure unit cell are much smaller than the illumination wavelength, so that the constructed material can be treated as a homogeneous medium characterized by averaged responses over the unit cell.

Metamaterials offer us more freedom to manipulate the propagation of light by achieving exotic material properties not found in conventional materials through artificially-designed 'metaatoms', especially in optical wavelengths, where metamaterials can manipulate the magnetic 
response incapable for conventional materials. So if an application is limited by conventional material responses, metamaterials might be a solution.

\subsection{Some Typical Potential Applications of Metamaterials and Their Limitations}

As metamaterials developed over time, numerous potential applications are proposed $[2,4-$ $8,12]$. In this section, only selected parts are chosen as examples to show the unique importance of metamaterials.

\subsubsection{Subwavelength imaging}

In imaging, rays emanating from an object can be divided into propagating and evanescent waves. For propagating waves, the wavevector component parallel to the lens $k_{\|}$is less than $k_{0}=\omega / c$, then the wavevector component perpendicular to the lens $k_{\perp}$ is real from the dispersion relation in vacuum $k_{\perp}^{2}+k_{\|}^{2}=k_{0}^{2}$. It is the opposite for evanescent waves, for which $k_{\|}>k_{0}$ and $k_{\perp}$ is imaginary. Hence, they decay exponentially with the distance from the object. For conventional positive- $n$ lenses, which require curved surfaces to focus light utilizing the refractive index contrast, propagating waves can be reassembled to a focus by applying a phase compensation. However, evanescent waves will be lost and their amplitudes can not be restored. Since evanescent waves carry finer details of an object, the focus with restored propagating waves only will have a maximum resolution around half of the illumination wavelength, i.e., the diffraction limit. This is a physical limit on a conventional lens, no matter how perfect the lens and how large its aperture. The situation changed when Pendry suggested a perfect lens [2] made of a flat NIM slab with $\varepsilon=-1, \mu=-1$, hence, $n=-1$ (see Figure 1.4). This perfect lens not only focuses propagating waves by a phase compensation, but also reproduces the same amplitude in the image plane as in the object plane by amplifying the field inside the lens. In Figure 1.4, we can see the evanescent near fields decay exponentially after emanating from the object, then exponentially grow in a NIM slab and exponentially decay again to the same amplitude in the image plane after exiting from the slab. Since all the 
rays emanating from an object can be restored on the image, ideally the perfect lens can have an unlimited resolution. Of course, it is impossible due to the inherent losses. However, it shows, in principle, subwavelength imaging is achievable and no fundamental physical reason prevents an image from a higher resolution beyond the diffraction limit. Subwavelength imaging can find a wide variety of potential applications, such as new devices on biomedical imaging with high resolution, subwavelength photolithography, nanocircuits, etc.

\subsubsection{Cloaking devices}
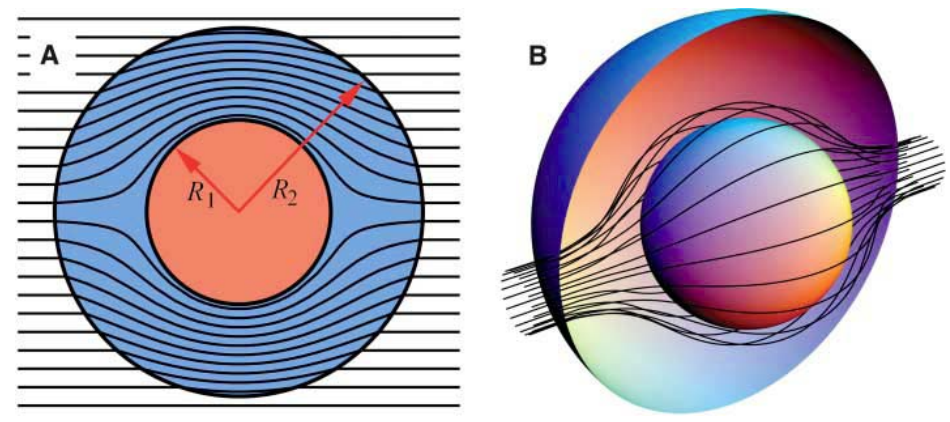

Figure 1.6 Illustration of the cloaking mechanism using metamaterials. (A) A 2D cross-section of rays trajectories in the cloaking system. (B) A 3D view. Figures are taken from Ref. [3]. The cloaked object (orange) is coated by a layer of metamaterials (blue), for which $\varepsilon$ and $\mu$ are spatially modulated, based on coordinate transformations [3].

Another active research area in metamaterials is the study of invisibility cloaking using metamaterial coating, based on coordinate transformations [3, 35-38]. It was first proposed by Pendry et al. in 2006 [3] and then experimentally demonstrated in the microwave frequencies [36] by Schurig et al. in the same year. The key idea is to exploit the flexibility of metamaterials to manipulate electromagnetic waves and produce new functionalities to take full control of the permittivity and permeability values throughout a material, independently taking any values as desired, such that the electromagnetic fields can be controlled to flow around a hidden object inside like a fluid and return to their original ray trajectories undisturbed on the far side of the object, which makes it appear nothing was there ever [3] (see Figure 1.6). Cloaking devices 
may have applications in stealth technology.

\subsubsection{Optical nonlinearity}

Metamaterials are also studied with the incorporation of conventional nonlinear materials $[6,12,39-43]$. Metamaterials can increase the interaction time with nonlinear medium by slowing light [44], and strongly enhance the local field [39, 40], hence they can enhance the nonlinear response $[12,39,40]$. The combination of metamaterials and nonlinear media has already been predicted to show some new properties, such as second-harmonic generation [43], bistability [40] and phase conjugation [42], which can be dynamically tuned by the light intensity of external stimuli. The combination of metamaterials and some conventional materials, such as liquid crystals [6], carbon nanotubes [41], conjugated polymers, semiconductors and semiconductor multiple-quantum well structures, can lead to fast and highly responsive nonlinearity in refractive index, $n$. Thus they are good candidates [12] to use in the application of nanoscale all-optical faster data processing. Zheludev [12] also suggested plasmonic nonlinearity of metals can be used together with metamaterials to achieve femtosecond timescale modulations on the propagating plasmon pulse [45], a speed at least five orders of magnitude faster than existing technologies.

\subsubsection{Zero index of refraction metamaterials}

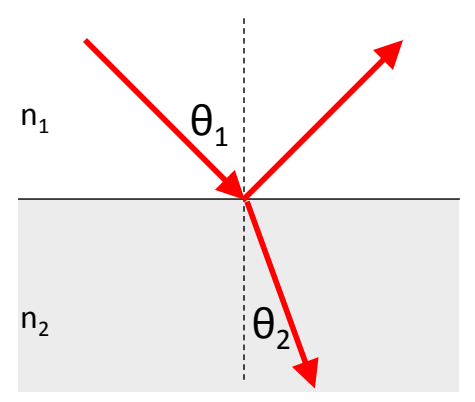

(a)

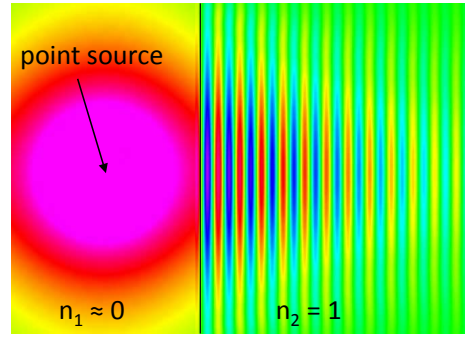

(b)

Figure 1.7 (a) Schematic of ray refractions at the material interface. (b) Rays emanating from a point source inside zero index metamaterials exit from the slab as a plane wave. 
Metamaterials can be engineered to have a zero index of refraction. For ray refractions occurring at the interface between two materials (see Figure 1.7(a)), assume the indice of refraction for these two materials are $n_{1}$ and $n_{2}$, respectively. The relation between the refracted angle, $\theta_{2}$, and the incident angle, $\theta_{1}$, can be obtained from Snell's law,

$$
n_{1} \sin \theta_{1}=n_{2} \sin \theta_{2}
$$

If we have $n_{1}=0$, then $\theta_{2}=0$, regardless the value of $\theta_{1}$. This means the rays emanating from a point source inside a metamaterial slab with zero index of refraction can switch to a plane wave after exiting from the slab. This idea can be used in beam generations and beam steering devices. It also suggests if we do the opposite, i.e., have a plane wave normally incident on the zero index metamaterial slab, the light can be focused. Hence, the metamaterial slab with zero index of refraction can work as a concentrator to help harvest sunlight in solar cells.

\subsubsection{The limitations on optical metamaterials}

Development of metamaterials has enabled scientists and engineers to work on applications in microwave regime. However, there are still limitations on optical metamaterials $[7,12]$ :

- large intrinsic losses,

- 3D isotropic designs,

- massive production of large-area structures.

This section focuses on the limitations from the large intrinsic losses in optical wavelengths, which have become the main obstacle towards real world applications for optical NIMs.

At microwave frequencies, the losses are small. For example, in Ref. [46], the transmission loss at the peak is the order of $1-5 \mathrm{~dB} / \lambda$. This is because the conductivity, $\sigma$, of a metal is extremely large so that it behaves like a perfect conductor. However, at optical wavelengths, $\varepsilon$ in metals can be very lossy, especially for nanostructures like thin films, where additional losses from electron scattering should be included [47, 48]. The large losses also come from 
the resonances in NIMs, in particular the magnetic resonance, needed to achieve negative $\mu$. Electric resonance can be avoided, because negative $\varepsilon$ can be obtained from the metallic background. These two factors make the losses of NIMs extremely large. So far, to the best of my knowledge, the best fabricated NIM at optical wavelengths is the fishnet structure from the Karlsruhe group operating at around $1.4 \mu \mathrm{m}$ wavelength [29], which shows a figure of merit $\mathrm{FOM}=|\operatorname{Re}(n) / \operatorname{Im}(n)|=3$. The corresponding absolute absorption coefficient for this FOM is $\alpha=3 \times 10^{4} \mathrm{~cm}^{-1}$, larger than the band-to-band absorption of typical direct-gap semiconductors such as, GaAs (where $\alpha=10^{4} \mathrm{~cm}^{-1}$ ). This can eliminate almost all envisioned applications of metamaterials at optical frequencies, except those designed to work as absorbers. For examples, the perfect lens has very strict requirements on material parameters [49, 50]. For a perfect lens with $\varepsilon=-1$ and $\mu=-1$, the transfer function should be unity for all $k_{\|}$. However, any deviation from $\varepsilon=-1$ and $\mu=-1$, including the inherent losses, even very small, can lead to an exponential decay of transfer function with $k_{\|}$. Hence, this limits the maximum $k_{\|}$for which the fields can be restored in the image plane $[49,50]$. Thus, the resolution is limited because high $k_{\|}$fields carry finer details of objects. This makes it impossible for existing optical NIMs to realize the perfect lens effect due to its large losses.

\subsection{The Methods to Reduce the Losses of Optical Metamaterials}

Although optical metamaterials have huge intrinsic losses, there is still a need to go into the optical region because they enable more exciting applications $[2,6,7,12]$. So, losses must be addressed. However, studies show that losses should decreases exponentially to obtain a linear improvement in the spatial resolution $[49,50]$. Hence, it is a big challenge to reduce the losses of optical metamaterials. In this section, some typical methods are presented to reduce the losses.

Indefinite media are referred as materials for which not all of the principal components of permittivity and permeability tensors have the same sign. Such materials were carefully studied by different groups [51-63]. It is shown that indefinite media can have negative group refraction, backward-wave effect, and partial focusing. The more interesting thing is that the evanescent 
near-fields can be converted to propagating fields inside an indefinite material slab, transferred to the other side of the slab, and refocused outside [51, 62]. Although there is no amplification inside the slab like a perfect lens, the evanescent waves can survive much longer distance than a conventional lens, and may provide subwavelength imaging at optical wavelengths. In addition, some dielectric indefinite media, such as a metallic-wire medium, do not need a resonance because we can obtain negative permittivity from the metallic background. So, these indefinite media do not have large resonance losses like ordinary NIMs and only the intrinsic metallic losses remain. By selecting low loss noble metals, such as silver and gold, the losses can be much lower than optical NIMs. We have conducted the theoretical study on optical 3D metallic-wire media made by silver and gold [62]. The results are presented in Chapter 3. Similar work was done by Liu [61]. Yao et al. [56] experimentally demonstrated negative refraction inside a silver-based wire medium at optical frequencies.

Geometry optimization is also used to reduce the losses. Zhou et al. [64] suggested the losses can be reduced by increasing the effective inductance to capacitance ratio, $L / C$, based on the fact that the damping factor, $\Gamma_{m}$, of a magnetic resonance, is inversely proportional to the effective inductance, $\Gamma_{m} \propto R / L$ ( $R$ is the effective resistance) [65]. By this approach, they numerically obtained a figure of merit FOM $=2.5$ for a fishnet structure at $484 \mathrm{~nm}$ wavelength, which is 5 times larger than the best result at $784 \mathrm{~nm}$. Zhou also suggested [66] a strong coupling between the fishnet functional layers can move the real part of the negative $n$ away from the region with the high imaginary part of $n$ so that one can obtain a high FOM value. This effect can also be seen in the experiment [28]. Guney et al. suggested [67] geometry tailoring to avoid the corners and sharp edges can lead to loss reductions.

Although losses can be reduced by geometry optimization discussed previously, achieving a significant enough loss reduction by further design optimization still appears out of reach. So far, the most promising way to reduce losses is incorporation of gain into metamaterial designs, first proposed by Ramakrishna and Pendry [68]. There are some issues necessary to be discussed here:

1. Quenching effect from contact with metals $[69,70]$. 
This quenching effect, in nature, is the photon-phonon interaction, which transfers electromagnetic energy non-radiatively to lattice vibrations and thus increases the loss of gain oscillators. So, it makes radiation less efficient. Experimentally, it has been shown the losses can be compensated in structures with metal-layer or metal-nanoparticle inclusions by organic semiconductor gain $[12,71,72]$. So, it is applicable to use gain to compensate the losses in the metamaterial nanostructures. In our simulations, gain and metal are separated by a dielectric spacer to avoid this effect.

2. Small amplification coefficients in bulk gain.

As discussed in section 1.2.5, the losses in NIMs are so large that its absorption coefficient $\left(\alpha>3 \times 10^{4} \mathrm{~cm}^{-1}\right)$ can be even larger than the band-to-band absorption of typical directgap semiconductors. Based on the assumption that the bulk gain coefficient is needed, it looks difficult to compensate the losses with gain, because it is not so easy to achieve the gain coefficient of this magnitude. Scientists [73] have shown what matters is the effective gain coefficient of the combined system instead of its bulk gain counterpart. Due to the strong local-field enhancement near metallic nanostructures, the effective gain coefficient can be substantially larger than the bulk gain.

3. Nonlinearity of gain.

The gain is nonlinear and its behavior depends on the optical intensity of the external incident light. Instead of naively forcing negative imaginary parts of the local gain material's response function to produce unrealistic strictly linear gain, we model the gain material using a semi-classical four-level atomic system and use a full-vectorial finite-difference time-domain approach to self-consistently couple the evolution of the occupation densities in the gain medium to Maxwell's equations. Nonlinearity, gain saturation, and Purcell effect are inherent in our model.

Many theoretical studies have been conducted on the loss compensation of optical metamaterials with gain in a variety of gain models $[68,74-83]$ and have shown the losses can 
be effectively compensated by gain. Recently, experimental progress has been made by some groups $[84,85]$, though some results are questioned.

\subsection{Overview and Layout}

The field of metamaterials has seen spectacular experimental progress in recent years and has been pushed into optical wavelengths. Optical metamaterials enables more exciting applications, including, but not limited to, superlens and all optical fast data processing. However, almost all these envisioned applications are limited by the huge loss of optical metamaterials. So far, the large intrinsic losses have become the major obstacle in the development of metamaterials. So, there is a need to address these losses. This thesis shows my effort in this topic.

A popular numerical tool for metamaterials is the finite-difference time-domain (FDTD) method, which discretizes Maxwell's equations in space and time. In Chapter 2, I give an introduction on this approach used in our numerical simulations. I discuss in detail the modeling of various materials used in the study of metamaterials, including lossy dielectrics, Lorentz media, gain materials and perfectly matched layer (PML). The gain is described by a generic four-level atomic system. Using this model, the FDTD approach can self-consistently couple the evolution of the occupation densities in the gain to Maxwell's equations. A parallel computational scheme is also presented to reduce the computation time and increase the effective usage of locally available resources. The errors at the discontinuous dielectric interface from the FDTD algorithm are analyzed by Taylor series expansions for 2D case. For the plane wave source, the total-field/scattered field $(\mathrm{TF} / \mathrm{SF})$ technique is used. Formulations in 2D case are presented. The detailed FDTD algorithms are given in appendix A.

Indefinite media are materials for which not all principal components of the permittivity and permeability tensors have the same sign. In Chapter 3, I design three-dimensional (3D) optical metallic nanowire media with different structures and numerically demonstrate that they can be homogeneous effective indefinite anisotropic media by showing that their dispersion relations are hyperbolic. For a finite slab, a nice fitting procedure is exploited to obtain the 
dispersion relations from which the effective permittivities are retrieved. Because the negative permittivity comes from the metallic background, no resonance is needed. Hence, the losses are comparatively small. The negative group refraction and pseudo focusing are observed. The pseudo focusing for the real 3D wire medium agrees very well with the homogeneous medium having the effective permittivity tensor of the wire medium. Studies also show that in the long-wavelength limit, the hyperbolic dispersion relation of the $3 \mathrm{D}$ wire medium can be valid even for evanescent modes, which may have important applications in super-resolution.

In Chapter 4, the self-consistent computational scheme presented in Chapter 2 is applied on systems of a dispersive metallic photonic metamaterial coupled to a gain material incorporated into the nanostructure. The lasing and nonlinear behaviors for gain slabs and a 1D layered metamaterial system alternating the layers of NIMs and gain materials are first studied. I also study the loss compensations at optical frequencies for various $2 \mathrm{D}$ and $3 \mathrm{D}$ metamaterial structures combined with gain, including a 2D lattice of resonant square cylinders embedded in layers of gain material, a lattice of 2D split ring resonators (SRRs) with gain material embedded into the gaps, a fishnet structure with gain embedded in-between two metal layers and an array of 3D SRRs with a gain layer underneath. Transmission, reflection, and absorption data as well as the retrieved effective parameters are presented. Studies show the losses can be effectively compensated by the electric gain in the studied cases. The effective gain coefficient of the combined system can be much larger than its bulk gain counterpart due to the strong local-field enhancement. 


\section{CHAPTER 2. THE FINITE DIFFERENCE TIME DOMAIN METHOD FOR ELECTROMAGNETICS}

\section{$2.1 \quad$ Introduction}

The finite difference time domain (FDTD) method is rapidly becoming one of the most popular numerical computational methods for the solution of electromagnetic problems since it was first proposed by Yee in 1966 [86]. Due to the reduction of computing costs, the simplicity of the method and high modeling capabilities of treating complex geometries, its popularity is

still growing. In addition, the extensions and enhancements to the method are continuously being proposed and published, which makes it more appealing.

For researchers in the area of metamaterials, the FDTD method is an essential and widely used simulation tool to quantify the electromagnetic properties of metamaterials. Nowadays there are lots of commercial FDTD software packages available for purchase in the market. And there are also some free software packages. However, the conventional FDTD software usually can not deal with all possible scenarios. Furthermore, the needs of research often demand the flexibility to modify the FDTD algorithms by access to the source codes, which is not offered by existing software packages. Hence, this prevents us from handling many problems of interest which fall beyond the capabilities of these existing packages.

To better understand the propagation of electromagnetic waves in metamaterials, I developed a modified FDTD algorithm suitable for modeling electromagnetic metamaterials. The FDTD code is capable of handling lossy dielectrics, lossy metals, dispersive media, left-handed materials (LHM), anisotropic materials and nonlinear active materials. The Maxwell's equations are discretized on a grid (Yee cell) both in time and space with a standard leap-frog in time, staggered grid scheme [86-88]. The electric field grid offsets both spatially and tem- 
porally from the magnetic field grid so that the present fields throughout the computational domain can be obtained in terms of the past fields [86-88]. In section 2.2, I present the FDTD electromagnetic formulations on modeling lossy dielectrics, Lorentz media, nonlinear active materials and perfectly matched layer (PML) absorbing media. A complete 3D FDTD update algorithm is given in appendix A. To simulate resonant phenomena like surface modes and finite size effects, large systems and high resolutions are necessary, however, this increases the computation resource requirements. One solution is to use parallel computation to solve a computational problem, which distributes the computing load into multiple CPUs, and hence reduces computation time. In section 2.3, a detailed parallel computation scheme is presented. In section 2.4, schemes on how to deal with the material interfaces are presented through error analysis. Finally, the 2D total-field/scattered-field (TF/SF) formulation used to realize an incident plane wave source is presented in section 2.5.

\subsection{Material Modeling}

\subsubsection{Lossy dielectrics}

For linear, nondispersive lossy dielectrics with dielectric constant $\varepsilon$ and conductivity $\sigma$, the Maxwell's equations can be written as follows:

$$
\begin{aligned}
\nabla \times \mathbf{E} & =-\mu_{0} \partial \mathbf{H} / \partial t \\
\nabla \times \mathbf{H} & =\varepsilon \varepsilon_{0} \partial \mathbf{E} / \partial t+\sigma \mathbf{E}+\mathbf{J}_{s}
\end{aligned}
$$

where $\mathbf{J}_{s}$ is electric source current density.

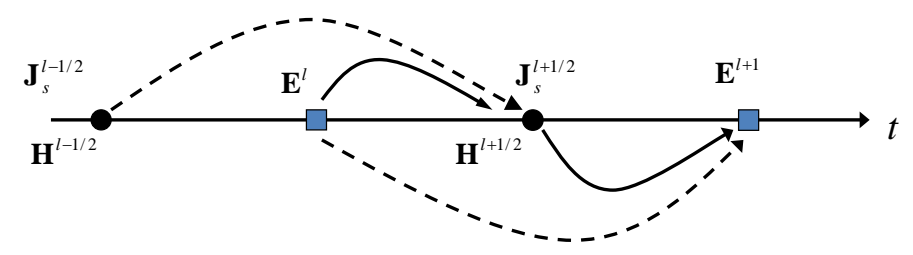

Figure 2.1 Schematic of the leap-frog scheme.

Using the leap-frog scheme [86-88] (see Figure 2.1), the central difference approximation 
and the notation $\mathbf{E}^{l}=\mathbf{E}(l \Delta t), \mathbf{H}^{l}=\mathbf{H}(l \Delta t)$ and $\mathbf{J}_{s}^{l}=\mathbf{J}_{s}(l \Delta t)$ ( $\Delta t$ is the time increment, assumed uniform over the observation interval), Eqs. 2.1 and 2.2 can be written in time-discrete form,

$$
\begin{gathered}
\mathbf{H}^{l+1 / 2}=\mathbf{H}^{l-1 / 2}-\frac{\Delta t}{\mu_{0}} \nabla \times \mathbf{E}^{l}, \\
\mathbf{E}^{l+1}=\frac{1-\sigma \Delta t / 2 \varepsilon \varepsilon_{0}}{1+\sigma \Delta t / 2 \varepsilon \varepsilon_{0}} \mathbf{E}^{l}+\frac{\Delta t / \varepsilon \varepsilon_{0}}{1+\sigma \Delta t / 2 \varepsilon \varepsilon_{0}}\left(\nabla \times \mathbf{H}^{l+1 / 2}-\mathbf{J}_{s}^{l+1 / 2}\right) .
\end{gathered}
$$

\subsubsection{Lorentz media}

Lorentz media are a very important class of material dispersions for FDTD modeling, which are typically characterized by the following dispersive permittivity,

$$
\varepsilon(\omega)=\varepsilon_{\infty}+\frac{\omega_{e p}^{2}}{\omega_{p}^{2}-i \omega \Gamma_{e}-\omega^{2}}
$$

Using time-domain auxiliary differential equation (ADE) method [87], the FDTD formulation for Lorentz media is developed below.

In time domain, Ampere's law can be expressed as

$$
\nabla \times \mathbf{H}(t)=\varepsilon_{0} \varepsilon_{\infty} \frac{\partial \mathbf{E}(t)}{\partial t}+\frac{\partial \mathbf{P}(t)}{\partial t}
$$

where $\mathbf{P}(t)$ is the electric polarization associated with the Lorentz resonance and the electric displacement field $\mathbf{D}$ is written as $\mathbf{D}=\varepsilon_{0} \varepsilon_{\infty} \mathbf{E}+\mathbf{P}$. The goal of the ADE technique is to develop a simple time-stepping scheme for $\mathbf{P}(t)$ which can be updated synchronously with Eq. 2.6. For

a monochromatic time harmonic electric field $\mathbf{E}=\mathbf{E}(\omega) e^{-i \omega t}$, we have $\mathbf{P}(\omega)=\varepsilon_{0} \chi_{e}(\omega) \mathbf{E}(\omega)$, where $\chi_{e}(\omega)$ is the susceptibility function and obtained for Lorentz media from Eq. 2.5 as follows,

$$
\chi_{e}(\omega)=\frac{\omega_{e p}^{2}}{\omega_{p}^{2}-i \omega \Gamma_{e}-\omega^{2}}
$$

Then,

$$
\mathbf{P}(\omega)=\frac{\varepsilon_{0} \omega_{e p}^{2}}{\omega_{p}^{2}-i \omega \Gamma_{e}-\omega^{2}} \mathbf{E}(\omega)
$$




$$
\omega_{p}^{2} \mathbf{P}(\omega)-i \omega \Gamma_{e} \mathbf{P}(\omega)-\omega^{2} \mathbf{P}(\omega)=\varepsilon_{0} \omega_{e p}^{2} \mathbf{E}(\omega)
$$

Conveniently exploiting the differentiation theorem for the Fourier transform, we perform an inverse Fourier transformation of each term of Eq. 2.9:

$$
\frac{\partial^{2} \mathbf{P}(t)}{\partial t^{2}}+\Gamma_{e} \frac{\partial \mathbf{P}(t)}{\partial t}+\omega_{p}^{2} \mathbf{P}(t)=\varepsilon_{0} \omega_{e p}^{2} \mathbf{E}(t)
$$

Eq. 2.10 can be written in a finite difference expression in time as follows,

$$
\frac{\mathbf{P}^{l+1}-2 \mathbf{P}^{l}+\mathbf{P}^{l-1}}{(\Delta t)^{2}}+\Gamma_{e} \frac{\mathbf{P}^{l+1}-\mathbf{P}^{l-1}}{2 \Delta t}+\omega_{p}^{2} \mathbf{P}^{l}=\varepsilon_{0} \omega_{e p}^{2} \mathbf{E}^{l} .
$$

Rewrite Eq. 2.11, we obtain

$$
\mathbf{P}^{l+1}=\alpha_{e} \mathbf{P}^{l}+\beta_{e} \mathbf{P}^{l-1}+\eta_{e} \mathbf{E}^{l}
$$

where

$$
\begin{aligned}
\alpha_{e} & =\frac{2-\omega_{p}^{2}(\Delta t)^{2}}{1+\Gamma_{e} \Delta t / 2}, \\
\beta_{e} & =-\frac{1-\Gamma_{e} \Delta t / 2}{1+\Gamma_{e} \Delta t / 2}, \\
\eta_{e} & =\frac{\varepsilon_{0} \omega_{e p}^{2}(\Delta t)^{2}}{1+\Gamma_{e} \Delta t / 2} .
\end{aligned}
$$

The polarization current can be written as $\mathbf{J}_{p}=\partial \mathbf{P} / \partial t$, then the time discretized form is

$$
\mathbf{J}_{p}^{l+1 / 2}=\frac{\mathbf{P}^{l+1}-\mathbf{P}^{l}}{\Delta t}
$$

Now, we can evaluate Eq. 2.6 at time step $l+1 / 2$ :

$$
\nabla \times \mathbf{H}^{l+1 / 2}=\varepsilon_{0} \varepsilon_{\infty}\left(\frac{\mathbf{E}^{l+1}-\mathbf{E}^{l}}{\Delta t}\right)+\mathbf{J}_{p}^{l+1 / 2} .
$$


Rewrite Eq. 2.15, we obtain the following explicit time-stepping expression for the E field,

$$
\mathbf{E}^{l+1}=\mathbf{E}^{l}+\frac{\Delta t}{\varepsilon_{0} \varepsilon_{\infty}}\left(\nabla \times \mathbf{H}^{l+1 / 2}-\mathbf{J}_{p}^{l+1 / 2}\right)
$$

For the $\mathbf{H}$ field, the time update expression is the same as Eq. 2.3. The ADE-FDTD algorithm for modeling a Lorentz medium needs to store $\mathbf{P}$ for two time-steps.

A special case of Lorentz media is the Drude dispersion, which has the resonance frequency $\omega_{p}=0$ in Eq. 2.5. Also Eq. 2.10 can be simplified as a first-order derivative equation by taking $\omega_{p}=0$ and $\mathbf{J}_{p}=\partial \mathbf{P} / \partial t$, which brings us an advantage that we directly get $\mathbf{J}_{p}$ and only need to store $\mathbf{J}_{p}$ for one time-step, and hence save the memory. For the electric polarization current update algorithm in time-discrete form, we have it as follows,

$$
\mathbf{J}_{p}^{l+1 / 2}=\frac{1-0.5 \Gamma_{e} \Delta t}{1+0.5 \Gamma_{e} \Delta t} \mathbf{J}_{p}^{l-1 / 2}+\frac{\varepsilon_{0} \omega_{e p}^{2} \Delta t}{1+0.5 \Gamma_{e} \Delta t} \mathbf{E}^{l}
$$

Similarly, for its magnetic counterpart of electric Lorentz dielectrics, it has magnetic permeability,

$$
\mu(\omega)=\mu_{\infty}+\frac{\omega_{m p}^{2}}{\omega_{p}^{2}-i \omega \Gamma_{m}-\omega^{2}} .
$$

Then, we can express Faraday's law in the time domain as

$$
\nabla \times \mathbf{E}=-\mu_{0} \mu_{\infty} \frac{\partial \mathbf{H}}{\partial t}-\frac{\partial \mathbf{M}}{\partial t}
$$

where $\mathbf{M}$ is the magnetic polarization. Following the similar procedure with electric Lorentz media, we can have the explicit time-stepping formulation for the magnetic polarization $\mathbf{M}$,

$$
\mathbf{M}^{l+3 / 2}=\alpha_{m} \mathbf{M}^{l+1 / 2}+\beta_{m} \mathbf{M}^{l-1 / 2}+\eta_{m} \mathbf{H}^{l+1 / 2},
$$

where

$$
\alpha_{m}=\frac{2-\omega_{p}^{2}(\Delta t)^{2}}{1+\Gamma_{m} \Delta t / 2},
$$




$$
\begin{aligned}
\beta_{m} & =-\frac{1-\Gamma_{m} \Delta t / 2}{1+\Gamma_{m} \Delta t / 2}, \\
\eta_{m} & =\frac{\mu_{0} \omega_{m p}^{2}(\Delta t)^{2}}{1+\Gamma_{m} \Delta t / 2}
\end{aligned}
$$

Similar to Eq. 2.14, the magnetic polarization current $\mathbf{K}_{m}$ is written as $\mathbf{K}_{m}=\partial \mathbf{M} / \partial t$ and its time-discrete form is

$$
\mathbf{K}_{m}^{l}=\frac{\mathbf{M}^{l+1 / 2}-\mathbf{M}^{l-1 / 2}}{\Delta t}
$$

Then the time-discrete form for Faraday's law is

$$
\mathbf{H}^{l+1 / 2}=\mathbf{H}^{l-1 / 2}-\frac{\Delta t}{\mu_{0} \mu_{\infty}}\left(\nabla \times \mathbf{E}^{l}+\mathbf{K}_{m}^{l}\right)
$$

Similarly, the corresponding magnetic Drude model when the resonance frequency $\omega_{p}=0$ in Eq. 2.18 has the magnetic current update algorithm as follows,

$$
\mathbf{K}_{m}^{l+1}=\frac{1-0.5 \Gamma_{m} \Delta t}{1+0.5 \Gamma_{m} \Delta t} \mathbf{K}_{m}^{l}+\frac{\mu_{0} \omega_{m p}^{2} \Delta t}{1+0.5 \Gamma_{m} \Delta t} \mathbf{H}^{l+\frac{1}{2}}
$$

\subsubsection{Nonlinear active materials}

In our model of active materials, the gain atoms are embedded in host medium and described by a generic four-level system [81-83, 89-91], as shown in Figure 2.2. All quantities including the fields and occupation numbers are tracked at each point in space and take into account energy exchange between gain atoms and fields, external pumping and non-radiative decays [89]. Electrons are pumped by an external mechanism from the ground state level $\left(N_{0}\right)$

to the third level $\left(N_{3}\right)$. After a short lifetime $\tau_{32}$, they quickly relax into the metastable second level $\left(N_{2}\right)$. The second level $\left(N_{2}\right)$ and the first level $\left(N_{1}\right)$ are called as the upper and lower lasing levels, respectively. Electrons can transfer both radiatively (spontaneous and stimulated emissions) and non-radiatively from the upper to the lower lasing level. At last, they transfer quickly and non-radiatively from the first level $\left(N_{1}\right)$ to the ground state level $\left(N_{0}\right)$. The energies of ground state and the third level are $E_{0}$ and $E_{3}$. In optical pumping mechanism, electrons are raised from the ground state level $\left(N_{0}\right)$ to the third level $\left(N_{3}\right)$ by an external 


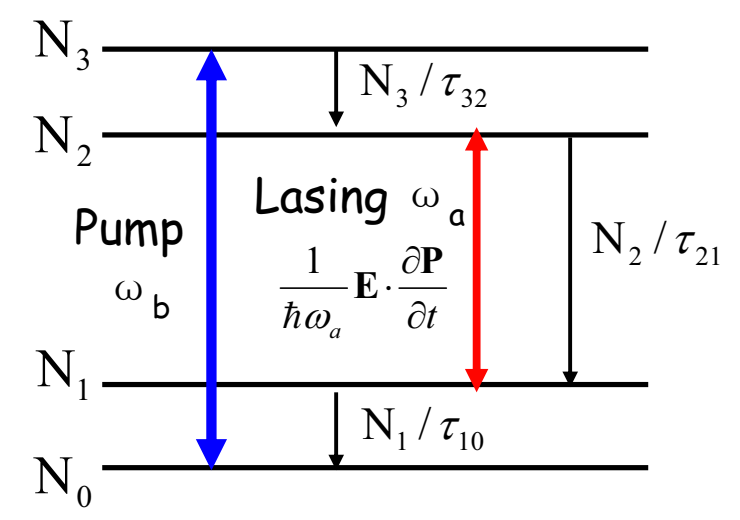

Figure 2.2 Schematic of the four-level atomic system model.

electromagnetic wave with the pumping frequency $\omega_{b}=\left(E_{3}-E_{0}\right) / \hbar$. The local intensity of the pumping EM wave varies with the position and determines the pumping rate at each point. The lifetimes and energies of the upper and lower lasing levels are $\tau_{21}, E_{2}$ and $\tau_{10}, E_{1}$, respectively. The center frequency of the radiation is $\omega_{a}=\left(E_{2}-E_{1}\right) / \hbar$. The total electron density, $N_{0}(t=0)=N_{0}(t)+N_{1}(t)+N_{2}(t)+N_{3}(t)$, does not vary with the time.

The time-dependent Maxwell's equations are given by

$$
\begin{aligned}
\nabla \times \mathbf{E} & =-\partial \mathbf{B} / \partial t \\
\nabla \times \mathbf{H} & =\varepsilon \varepsilon_{o} \partial \mathbf{E} / \partial t+\partial \mathbf{P} / \partial t
\end{aligned}
$$

where $\mathbf{B}=\mu \mu_{o} \mathbf{H}$ and $\mathbf{P}=\sum_{i=a, b} \mathbf{P}_{i}$ is the electric polarization density of the gain material. $\left(\mathbf{P}_{a}\right.$ is the induced electric polarization density on the atomic transition between the upper $\left(N_{2}\right)$ and lower $\left(N_{1}\right)$ lasing levels, and $\mathbf{P}_{b}$ is between the ground state level $\left(N_{0}\right)$ and the third level $\left(N_{3}\right)$.) The induced electric polarizations behave as harmonic oscillators and couple to the local $\mathbf{E}$ field, which is propagated by Maxwell's equations. The polarization density $\mathbf{P}_{i}(\mathbf{r}, t)$ obeys locally the following equation of motion [89],

$$
\frac{\partial^{2} \mathbf{P}_{i}(t)}{\partial t^{2}}+\Gamma_{i} \frac{\partial \mathbf{P}_{i}(t)}{\partial t}+\omega_{i}^{2} \mathbf{P}_{i}(t)=-\sigma_{i} \Delta N_{i}(t) \mathbf{E}(t) \quad(i=a, b)
$$

where $\Gamma_{i}$ is the linewidth of the atomic transition $\omega_{i}, \sigma_{i}$ is the coupling strength of $\mathbf{P}_{i}$ to the 
electric field, and $\Delta N_{a}(\mathbf{r}, t)=N_{2}(\mathbf{r}, t)-N_{1}(\mathbf{r}, t)$ and $\Delta N_{b}(\mathbf{r}, t)=N_{3}(\mathbf{r}, t)-N_{0}(\mathbf{r}, t)$ are the population inversions that drive the polarizations. From Eq. 2.26, it can be easily derived [89] that the atomic response of gain atoms has a Lorentz lineshape and is homogeneously broadened. The occupation numbers at each spatial point vary according to the following rate equations,

$$
\begin{aligned}
\frac{\partial N_{3}}{\partial t} & =\frac{1}{\hbar \omega_{b}} \mathbf{E} \cdot \frac{\partial \mathbf{P}_{b}}{\partial t}-\frac{N_{3}}{\tau_{32}}, \\
\frac{\partial N_{2}}{\partial t} & =\frac{N_{3}}{\tau_{32}}+\frac{1}{\hbar \omega_{a}} \mathbf{E} \cdot \frac{\partial \mathbf{P}_{a}}{\partial t}-\frac{N_{2}}{\tau_{21}}, \\
\frac{\partial N_{1}}{\partial t} & =\frac{N_{2}}{\tau_{21}}-\frac{1}{\hbar \omega_{a}} \mathbf{E} \cdot \frac{\partial \mathbf{P}_{a}}{\partial t}-\frac{N_{1}}{\tau_{10}}, \\
\frac{\partial N_{0}}{\partial t} & =-\frac{1}{\hbar \omega_{b}} \mathbf{E} \cdot \frac{\partial \mathbf{P}_{b}}{\partial t}+\frac{N_{1}}{\tau_{10}},
\end{aligned}
$$

where $\frac{1}{\hbar \omega_{i}} \mathbf{E} \cdot \frac{\partial \mathbf{P}_{i}}{\partial t}(i=a, b)$ is the induced radiation rate or excitation rate depending on its sign.

Instead of using an external EM wave to optically pump electrons from the ground state level $\left(N_{0}\right)$ to the third level $\left(N_{3}\right)$, we can simplify this process in Eqs. $2.27 \mathrm{a}$ and $2.27 \mathrm{~d}$ by pumping electrons with a homogeneous pumping rate $\Gamma_{\text {pump }}$, which is proportional to the optical pumping intensity in an experiment. This simplification is valid only if the gain slab is thin and the gain of the laser is low, because the real pumping rate depends on the local optical intensity and should be a function of position. We will discuss this in more detail in Chapter 4. Based on this simplification, we can have the rate equations as follows,

$$
\begin{aligned}
& \frac{\partial N_{3}}{\partial t}=\Gamma_{\text {pump }} N_{0}-\frac{N_{3}}{\tau_{32}}, \\
& \frac{\partial N_{2}}{\partial t}=\frac{N_{3}}{\tau_{32}}+\frac{1}{\hbar \omega_{a}} \mathbf{E} \cdot \frac{\partial \mathbf{P}_{a}}{\partial t}-\frac{N_{2}}{\tau_{21}}, \\
& \frac{\partial N_{1}}{\partial t}=\frac{N_{2}}{\tau_{21}}-\frac{1}{\hbar \omega_{a}} \mathbf{E} \cdot \frac{\partial \mathbf{P}_{a}}{\partial t}-\frac{N_{1}}{\tau_{10}}, \\
& \frac{\partial N_{0}}{\partial t}=\frac{N_{1}}{\tau_{10}}-\Gamma_{\text {pump }} N_{0} .
\end{aligned}
$$

Correspondingly, we only need to consider the electric polarization density $\mathbf{P}_{a}(\mathbf{r}, t)$ on the atomic transition between $N_{2}$ and $N_{1}$ in Eqs. 2.25 and 2.26 . 
Assuming the three electric oscillators along $x, y$ and $z$ directions are independent and taking the leap-frog scheme in time, we can find the explicit time-stepping expression for the driven oscillators in Eq. 2.26,

$$
\begin{aligned}
& P_{a j}^{l+1}=\frac{2-\omega_{a}^{2}(\Delta t)^{2}}{1+\Gamma_{a} \Delta t / 2} P_{a j}^{l}-\frac{1-\Gamma_{a} \Delta t / 2}{1+\Gamma_{a} \Delta t / 2} P_{a j}^{l-1}+\frac{\sigma_{a}(\Delta t)^{2}}{1+\Gamma_{a} \Delta t / 2}\left(N_{1 j}^{l}-N_{2 j}^{l}\right) E_{j}^{l}, \\
& P_{b j}^{l+1}=\frac{2-\omega_{b}^{2}(\Delta t)^{2}}{1+\Gamma_{b} \Delta t / 2} P_{b j}^{l}-\frac{1-\Gamma_{b} \Delta t / 2}{1+\Gamma_{b} \Delta t / 2} P_{b j}^{l-1}+\frac{\sigma_{b}(\Delta t)^{2}}{1+\Gamma_{b} \Delta t / 2}\left(N_{0 j}^{l}-N_{3 j}^{l}\right) E_{j}^{l},
\end{aligned}
$$

where $j=x, y, z$, denoting the corresponding component in each direction. $N_{0 j}, N_{1 j}, N_{2 j}$, and $N_{3 j}$ are the occupation numbers in different levels for the corresponding independent electric gain oscillator in $j$ direction. For the simplification using a homogeneous pumping rate, we only need to update $\mathbf{P}_{a}$. The electric polarization current can be written as $\mathbf{J}_{p}=\partial \mathbf{P} / \partial t$. Then we can have the following time-discrete forms,

$$
\begin{aligned}
& \mathbf{J}_{p}^{l+1 / 2}=\frac{\mathbf{P}_{a}^{l+1}-\mathbf{P}_{a}^{l}}{\Delta t}+\frac{\mathbf{P}_{b}^{l+1}-\mathbf{P}_{b}^{l}}{\Delta t} \quad \text { (Optical pumping) }, \\
& \mathbf{J}_{p}^{l+1 / 2}=\frac{\mathbf{P}_{a}^{l+1}-\mathbf{P}_{a}^{l}}{\Delta t} \quad \text { (Homogeneous pumping rates) } .
\end{aligned}
$$

Using the electric polarization current $\mathbf{J}_{p}=\partial \mathbf{P} / \partial t$, Eq. 2.25 can be discretized in time,

$$
\begin{aligned}
\mathbf{H}^{l+1 / 2} & =\mathbf{H}^{l-1 / 2}-\frac{\Delta t}{\mu_{0} \mu} \nabla \times \mathbf{E}^{l}, \\
\mathbf{E}^{l+1} & =\mathbf{E}^{l}+\frac{\Delta t}{\varepsilon \varepsilon_{0}}\left(\nabla \times \mathbf{H}^{l+1 / 2}-\mathbf{J}_{p}^{l+1 / 2}\right) .
\end{aligned}
$$

The driven oscillators are coupled to the rate equations by the population inversions $\Delta N$. Following the leap-frog scheme in time, the rate equations Eq. 2.27 for optical pumping can be written in a time-discrete form,

$$
\begin{aligned}
& \frac{N_{3 j}^{l+1}-N_{3 j}^{l}}{\Delta t}=\frac{1}{\hbar \omega_{b}} \frac{E_{j}^{l+1}+E_{j}^{l}}{2} \frac{P_{b j}^{l+1}-P_{b j}^{l}}{\Delta t}-\frac{N_{3 j}^{l+1}+N_{3 j}^{l}}{2 \tau_{32}}, \\
& \frac{N_{2 j}^{l+1}-N_{2 j}^{l}}{\Delta t}=\frac{N_{3 j}^{l+1}+N_{3 j}^{l}}{2 \tau_{32}}+\frac{1}{\hbar \omega_{a}} \frac{E_{j}^{l+1}+E_{j}^{l}}{2} \frac{P_{a j}^{l+1}-P_{a j}^{l}}{\Delta t}-\frac{N_{2 j}^{l+1}+N_{2 j}^{l}}{2 \tau_{21}} \\
& \frac{N_{1 j}^{l+1}-N_{1 j}^{l}}{\Delta t}=\frac{N_{2 j}^{l+1}+N_{2 j}^{l}}{2 \tau_{21}}-\frac{1}{\hbar \omega_{a}} \frac{E_{j}^{l+1}+E_{j}^{l}}{2} \frac{P_{a j}^{l+1}-P_{a j}^{l}}{\Delta t}-\frac{N_{1 j}^{l+1}+N_{1 j}^{l}}{2 \tau_{10}}
\end{aligned}
$$




$$
\frac{N_{0 j}^{l+1}-N_{0 j}^{l}}{\Delta t}=-\frac{1}{\hbar \omega_{b}} \frac{E_{j}^{l+1}+E_{j}^{l}}{2} \frac{P_{b j}^{l+1}-P_{b j}^{l}}{\Delta t}+\frac{N_{1 j}^{l+1}+N_{1 j}^{l}}{2 \tau_{10}}
$$

where $j=x, y, z$. Upon collecting like terms, we can obtain the explicit time-stepping relation for occupation numbers,

$$
\begin{aligned}
N_{3 j}^{l+1}= & \frac{1-\Delta t / 2 \tau_{32}}{1+\Delta t / 2 \tau_{32}} N_{3 j}^{l}+\frac{1}{2 \hbar \omega_{b}} \frac{1}{1+\Delta t / 2 \tau_{32}}\left(E_{j}^{l+1}+E_{j}^{l}\right)\left(P_{b j}^{l+1}-P_{b j}^{l}\right), \\
N_{2 j}^{l+1}= & \frac{1-\Delta t / 2 \tau_{21}}{1+\Delta t / 2 \tau_{21}} N_{2 j}^{l}+\frac{1}{2 \hbar \omega_{a}} \frac{1}{1+\Delta t / 2 \tau_{21}}\left(E_{j}^{l+1}+E_{j}^{l}\right)\left(P_{a j}^{l+1}-P_{a j}^{l}\right) \\
& +\frac{1}{1+\Delta t / 2 \tau_{21}} \frac{\Delta t}{2 \tau_{32}}\left(N_{3 j}^{l+1}+N_{3 j}^{l}\right), \\
N_{1 j}^{l+1}= & \frac{1-\Delta t / 2 \tau_{10}}{1+\Delta t / 2 \tau_{10}} N_{1 j}^{l}-\frac{1}{2 \hbar \omega_{a}} \frac{1}{1+\Delta t / 2 \tau_{10}}\left(E_{j}^{l+1}+E_{j}^{l}\right)\left(P_{a j}^{l+1}-P_{a j}^{l}\right) \\
& +\frac{1}{1+\Delta t / 2 \tau_{10}} \frac{\Delta t}{2 \tau_{21}}\left(N_{2 j}^{l+1}+N_{2 j}^{l}\right), \\
N_{0 j}^{l+1}= & N_{0 j}^{l}-\frac{1}{2 \hbar \omega_{b}}\left(E_{j}^{l+1}+E_{j}^{l}\right)\left(P_{b j}^{l+1}-P_{b j}^{l}\right)+\frac{\Delta t}{2 \tau_{10}}\left(N_{1 j}^{l+1}+N_{1 j}^{l}\right) .
\end{aligned}
$$

For the simplification using a homogeneous pumping rate, Eqs. 2.33b and 2.33c keep the same, and Eqs. 2.33a and 2.33d are changed to the following,

$$
\begin{aligned}
& N_{3 j}^{l+1}=\frac{1-\Delta t / 2 \tau_{32}}{1+\Delta t / 2 \tau_{32}} N_{3 j}^{l}+\frac{\Delta t}{1+\Delta t / 2 \tau_{32}} \Gamma_{\text {pump }} N_{0 j}^{l}, \\
& N_{0 j}^{l+1}=\left(1-\Gamma_{\text {pump }} \Delta t\right) N_{0 j}^{l}+\frac{\Delta t}{2 \tau_{10}}\left(N_{1 j}^{l+1}+N_{1 j}^{l}\right) .
\end{aligned}
$$

Note: from the FDTD update algorithms for the occupation numbers in Eqs. 2.33 and 2.34, we can see the update of these occupation numbers should be done in the following order: $N_{3}$, $N_{2}, N_{1}$ and $N_{0}$.

\subsubsection{Perfectly matched layer (PML) absorbing boundary conditions}

In numerical simulations, lots of geometries of interest are defined in "open" regions where the spatial domain of the computed field is unbounded in one or more dimensions. Due to the limitation of time and computation resources, it is impossible to handle such an unbounded region problem directly. So there is a need to introduce an absorbing boundary condition (ABC) 
at the outer lattice boundary to simulate the extension of the FDTD computation domain to infinity.

There are two different categories for absorbing boundaries: one is derived from differential equations, such as Mur's [87, 92] and Liao's [87, 93] absorbing boundary conditions; another one is actually not a real "boundary" condition, instead, it terminates the outer boundary of the space lattice by surrounding the computation domain with a lossy, reflectionless material which damps the outgoing fields [87]. In the second category, the perfectly matched layer (PML) technique shows much more accuracy than other ABCs. It's only a few lattice thick, reflectionless to all impinging waves (arbitrary incidence and polarization) over their full frequency spectrum and highly absorbing. Here we present the PML technique proposed by J. P. Berenger [94], who derived a novel split-field formulation of Maxwell's equations where each vector field component is split into two orthogonal components [87, 94].

For 3D case, the six electric and magnetic field components yield 12 subcomponents, denoted by $E_{x y}, E_{x z}, E_{y x}, E_{y z}, E_{z x}, E_{z y}, H_{x y}, H_{x z}, H_{y x}, H_{y z}, H_{z x}$ and $H_{z y}$ in Cartesian coordinates. Using these subcomponents, we have 3D time-domain Maxwell's equations for Berenger's split-fields [87, 94],

$$
\begin{aligned}
& \left(\varepsilon \frac{\partial}{\partial t}+\sigma_{y}\right) E_{x y}=\frac{\partial H_{z}}{\partial y}, \\
& \left(\varepsilon \frac{\partial}{\partial t}+\sigma_{z}\right) E_{x z}=-\frac{\partial H_{y}}{\partial z}, \\
& \left(\varepsilon \frac{\partial}{\partial t}+\sigma_{z}\right) E_{y z}=\frac{\partial H_{x}}{\partial z}, \\
& \left(\varepsilon \frac{\partial}{\partial t}+\sigma_{x}\right) E_{y x}=-\frac{\partial H_{z}}{\partial x}, \\
& \left(\varepsilon \frac{\partial}{\partial t}+\sigma_{x}\right) E_{z x}=\frac{\partial H_{y}}{\partial x}, \\
& \left(\varepsilon \frac{\partial}{\partial t}+\sigma_{y}\right) E_{z y}=-\frac{\partial H_{x}}{\partial y}, \\
& \left(\mu \frac{\partial}{\partial t}+\sigma_{y}^{*}\right) H_{x y}=-\frac{\partial E_{z}}{\partial y}, \\
& \left(\mu \frac{\partial}{\partial t}+\sigma_{z}^{*}\right) H_{x z}=\frac{\partial E_{y}}{\partial z}, \\
& \left(\mu \frac{\partial}{\partial t}+\sigma_{z}^{*}\right) H_{y z}=-\frac{\partial E_{x}}{\partial z},
\end{aligned}
$$




$$
\begin{aligned}
& \left(\mu \frac{\partial}{\partial t}+\sigma_{x}^{*}\right) H_{y x}=\frac{\partial E_{z}}{\partial x} \\
& \left(\mu \frac{\partial}{\partial t}+\sigma_{x}^{*}\right) H_{z x}=-\frac{\partial E_{y}}{\partial x} \\
& \left(\mu \frac{\partial}{\partial t}+\sigma_{y}^{*}\right) H_{z y}=\frac{\partial E_{x}}{\partial y}
\end{aligned}
$$

where we have the following relations:

$$
\begin{aligned}
& E_{x}=E_{x y}+E_{x z}, \\
& E_{y}=E_{y x}+E_{y z}, \\
& E_{z}=E_{z x}+E_{z y}, \\
& H_{x}=H_{x y}+H_{x z}, \\
& H_{y}=H_{y x}+H_{y z}, \\
& H_{z}=H_{z x}+H_{z y}
\end{aligned}
$$

and the parameters $\sigma_{x}, \sigma_{y}, \sigma_{z}, \sigma_{x}^{*}, \sigma_{y}^{*}$ and $\sigma_{z}^{*}$ are electric and magnetic conductivities.

J. P. Berenger has shown that any outgoing waves from the inner lossless isotropic medium can penetrate without reflection into these unphysical absorbing layers and get highly absorbed, independent of the frequency and the angle of incidence, if the absorbing media satisfy the following matching conditions[87, 94]:

a. at a normal-to- $w(w=x, y, z)$ PML interface in the FDTD lattice, the parameter pair $\left(\sigma_{w}, \sigma_{w}^{*}\right)$ satisfies $\sigma_{w}^{*} / \mu=\sigma_{w} / \varepsilon$ and all other $\left(\sigma_{w}, \sigma_{w}^{*}\right)$ pairs are zero. The permittivity $\varepsilon$ and permeability $\mu$ of PML should be the same as the inner medium;

b. in a corner region, the PML is provided with each matched $\left(\sigma_{w}, \sigma_{w}^{*}\right)$ pair that is assigned to the overlapping PMLs forming the corner. So, PML media located in dihedral-corner overlapping regions have two nonzero and one zero $\left(\sigma_{w}, \sigma_{w}^{*}\right)$ pairs. And PML media located in trihedral-corner overlapping regions have three nonzero $\left(\sigma_{w}, \sigma_{w}^{*}\right)$ pairs.

The time-discrete form of Eq. 2.35 can be easily obtained by applying the central difference approximation to the temporal and spatial partial differential operator. Please see appendix 
A for the update FDTD algorithms in detail.

Theoretically, reflectionless wave transmission can occur across a PML interface regardless of the local step-discontinuity in $\sigma$ and $\sigma^{*}$ in continuous media. However, in FDTD or any discrete representation of Maxwell's equations, due to the numerical artifacts caused by spatial discretization, the step-discontinuities in $\sigma$ and $\sigma^{*}$ at the PML surface can lead to significant discretization error that is manifested as a spurious reflection. To reduce this reflection error, J. P. Berenger proposed to spatially grade the conductivity profile along the normal axis $[87,88,94]$. Here we use a polynomial grading of the PML loss with depth $x[87,88,95]$,

$$
\sigma_{x}=(x / d)^{m} \sigma_{x, \max }
$$

where $\sigma_{x, \max }=-\frac{(m+1) \ln [R(0)]}{2 \eta d}, d$ is the thickness of PML, and $\eta$ is the EM impedance in inner medium and $R[0]$ is the desired reflection error. Eq. 2.37 increases the value of $\sigma_{x}$ from zero at $x=0$, the PML surface, to $\sigma_{x, \max }$ at $x=d$, the outer boundary of PML. Typically, it has been found that $m$ in the range between 3 and 4 is almost optimal for many FDTD simulations [87, 88, 95-98].

Although J. P. Berenger's split-field PML is very robust and efficient for the termination of FDTD lattices, it can not absorb evanescent waves [96, 99-102]. For wave-structure interaction problems, evanescent fields are usually present in the region of interest. Hence the numerical reflection of evanescent fields may lead to significant numerical errors. In some circumstances, it can even result in numerical instability. One way to prevent this problem is to place the PML sufficiently far away from the structure such that the evanescent waves have sufficiently decayed. This method requires a lot of computation resources. Another way is to take improved/novel PML techniques [96, 100-102], which allow a substantial absorption of evanescent waves such that the structure-PML separation can be much shorter. 


\subsection{The Parallel Computation Scheme}

In plasmonics and metamaterials, resonant problems like surface modes and finite size effects are a very important region of interest. To study these problems, large systems and high resolutions are necessary, which may cost a lot of memories and computation time. However, the size of memory for each CPU is limited by the operating system. To mitigate this problem, parallel computation is used, which distributes the computing load into multiple CPUs, and hence increases the available computation space (e.g., 256 million grid cells on 64 CPUs for a 2D case in our cluster) and reduces computation time. As a result, local resources can be effectively used.
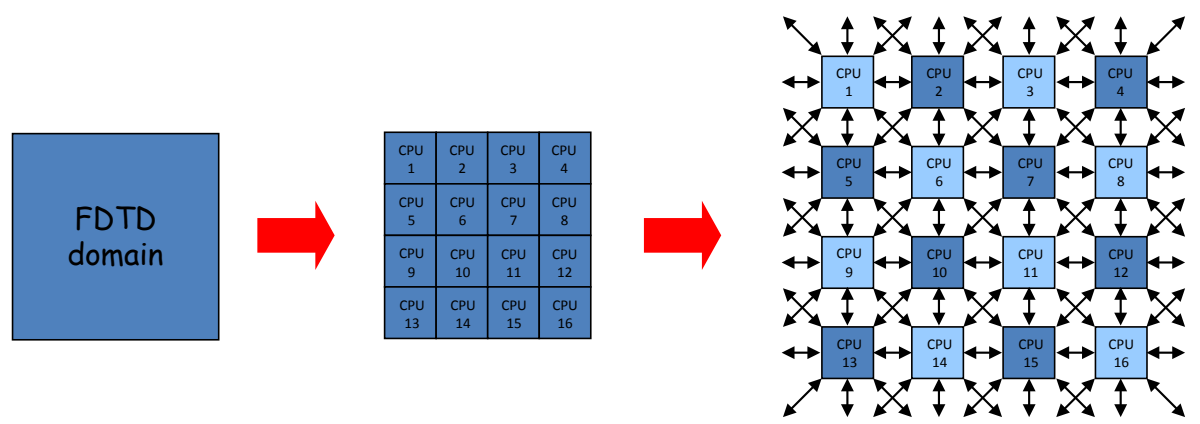

Figure 2.3 Schematic of the parallel computation scheme.

The capability of the parallel computation for FDTD simulations is due to the intrinsic characteristic of the update algorithms which only need the fields in the neighboring Yee cells. Figure 2.3 shows our parallel computation scheme for FDTD simulations. The FDTD computation domain is split into many blocks and each is assigned to one CPU. Each CPU runs serially the same update algorithms inside the block and communicates every time-step with its neighboring CPUs to send and receive field data in the block edges. Technically, for edge data communications, each computation block/domain is extended by a foreign edge buffer,the gray area shown in Figure 2.4. The update only runs in the domain interior (blue area) and the field data in the inner edge zone are sent to the foreign edge buffers of its neighboring domains. The foreign edge buffer receives and stores the field data from neighboring domain inner edge zones, which can be used in updating the fields in the inner edge zone of the domain 
itself.

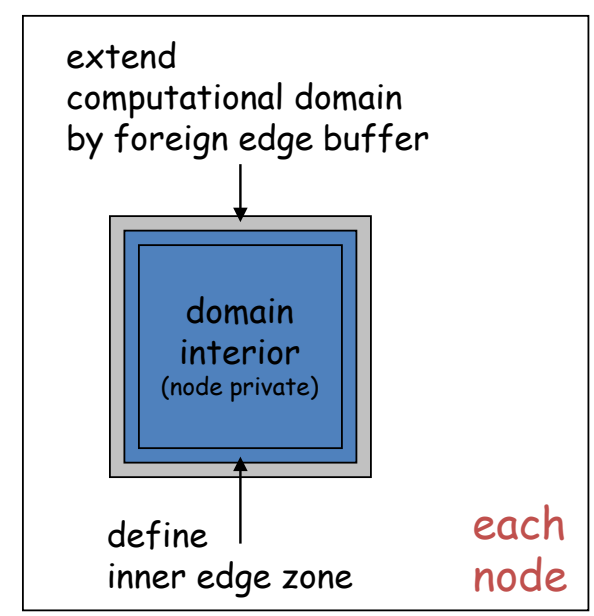

Figure 2.4 Illustration of the extension of each computation domain by a foreign edge buffer to communicate the edge field data.

The message passing between different nodes in our code is realized by using the MPI library as the communication layer. Here we have to pay attention to the message passing order between nodes, because having improper message passing order can cause "deadlock" of the code implementation. As an example shown in Figure 2.5, the implementation of A needs to wait for the completion of $\mathrm{D}$, however, $\mathrm{D}$ needs to wait for $\mathrm{B}$. Meanwhile, $\mathrm{B}$ needs to wait for $\mathrm{C}$ and $\mathrm{C}$ needs to wait for A. Then the implementation falls in a "deadlock" and can not continue. It is also not good to have all the nodes send/write the message first and then receive/read the message, because sending the message out needs the buffer allocation from

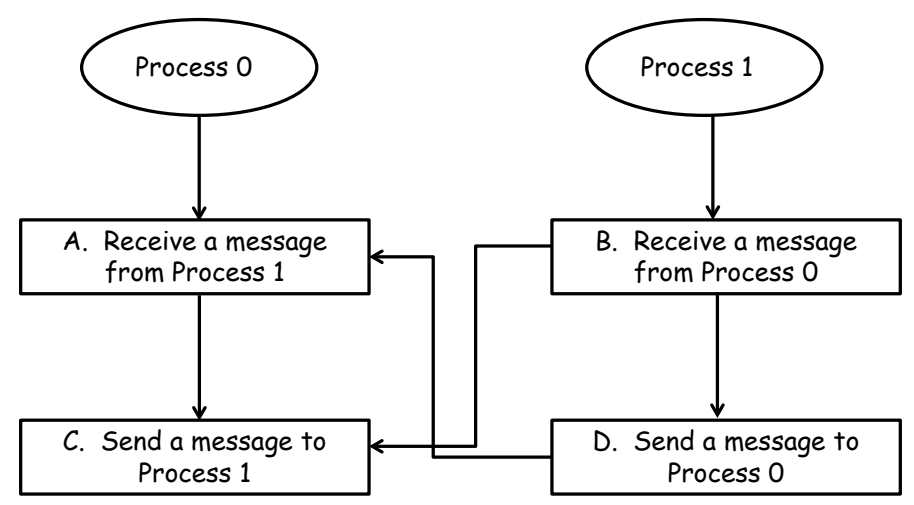

Figure 2.5 Improper message passing order leading to deadlock. 
OS and if OS can not provide sufficient buffers, then the sending can not be finished.
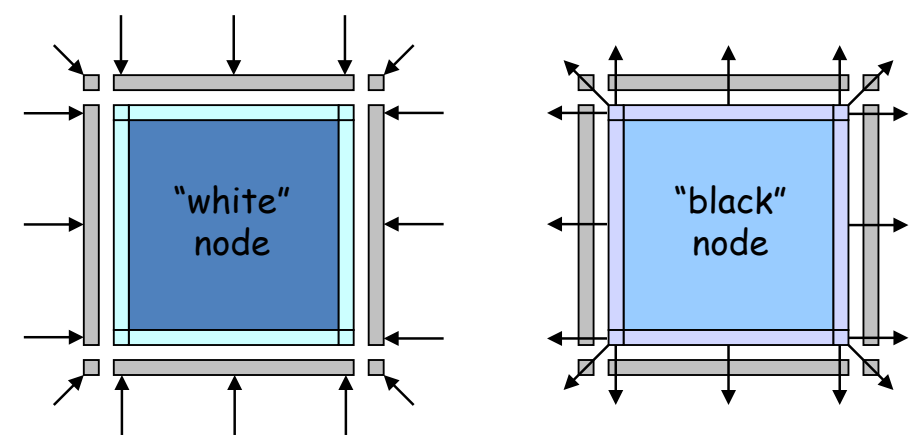

Figure 2.6 The communication scheme between neighboring nodes.

To safely communicate between nodes, we divide the split computation domain into "white" nodes and "black" nodes (see Figure 2.6), where the "black" ones send/write first while the "white" ones receive/read first. As an example, to communicate the field data in the vertical edges of the split computation domain in Figure 2.3, we alternate the blocks with the "white" node and the "black" node in the horizontal direction, i.e., the nodes in even columns are "white" nodes, while those in odd columns are "black" nodes. First the "black" nodes send the field data in the right vertical edges to the "white" nodes, which receive the data and store them in the left sides of their foreign edge buffers. Then the "black" ones send the left vertical edge data and the "white" ones store them in the right sides of the foreign edge buffers. After that, the even and odd columns exchange the color and repeat the same procedure. As a result, all vertical edge data are passed to their corresponding neighboring nodes. For the horizontal edge data, the communication can be done by repeating the above procedure. To communicate the field data in the corners, we can alternate the blocks either in the horizontal direction or the vertical direction, then the "black" nodes send the corner field data clockwisely to their diagonal neighboring nodes and the "white" nodes receive these data clockwisely and store them in the corresponding corners of the foreign buffers. For 3D case, we can follow the similar procedure to finish the edge data communication in three dimensions. The difference is that we need to communicate the data for 6 face edges, 12 dihedral corners and 8 trihedral corners in $3 \mathrm{D}$ case. 
For a computation domain split into $m$ blocks and each with $n \times n \times n$ cells, the computation time can be given by

$$
t_{p} \propto n^{3}+12 n^{2}+24 n
$$

where $n^{3}$ comes from the FDTD updates in the block interior and $12 n^{2}+24 n$ comes from the

edge field data communication. In the serial computation, the computation time is $t_{s} \propto m \cdot n^{3}$. From these two computation times, we can obtain the ratio of them to measure how effective the parallel computation is,

$$
\frac{t_{p}}{t_{s}}=\frac{1+12 / n+24 / n^{2}}{m} .
$$

Eq. 2.39 shows the relative overhead of edge communications is roughly inversely proportional to linear dimension $n$ and the parallel computation gets more effective with increasing $n$. For a large enough subdomain size, the relative overhead of edge communications is very small so that we can still have high effective parallel computations with only moderate fast switched network interconnect. In real implementations, the block may not be a cube. In order to reduce the relative overhead of edge communications and keep the parallel computation highly effective, we have to have the block as cubic as possible.

\subsection{Error Analysis at Material Interfaces}

Generally, we use the Taylor series expansion to determine the order of accuracy of FDTD algorithms. As known, a second-order accuracy can be obtained for the spatially-extended space derivative operators in the regular Yee scheme, which assume the materials are homogeneous, at least within the extent of their stencil. However, due to the inhomogeneity of the coefficients in Maxwell's equations, this full accuracy can not be realized across discontinuous material interfaces and will be degraded to first order [103]. In this section, the error analysis is presented for the regular Yee scheme at dielectric interfaces in 2D case. 


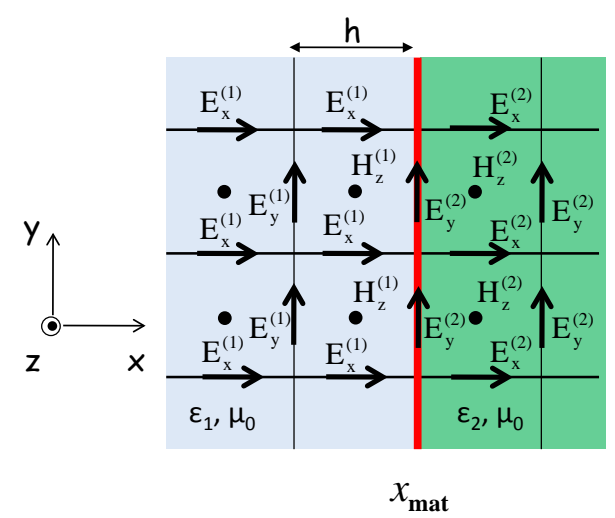

Figure 2.7 Illustration of Yee grids in the TM case. In the light blue and green area, the dielectric constants are $\varepsilon_{1}$ and $\varepsilon_{2}$, respectively. Both of them have the permeability $\mu=\mu_{0}$. The red line at the position $x=x_{\text {mat }}$ is the material interface. The Yell cell size is $h \times h$.

\subsubsection{TM case}

Here we define the TM modes as the modes with the field components $E_{x}, E_{y}$ and $H_{z}$ only. To simplify the discussion, we have the material interface aligned with the electric field link of the Yee cell, as shown in Figure 2.7. This can also have an advantage that the continuity of the tangential field components can be guaranteed along the interface. For the sake of the discussion, we introduce two sets of fields, $\left(E^{(k)}, H^{(k)}\right)$ with $k=1,2$, representing the solutions in the two different material regions (see Figure 2.7). These two solutions are connected by the boundary conditions that the tangential field components are continuous across the interface. We also assume the interface has the dielectric constant $\varepsilon_{2}$ and the solution $\left(E^{(2)}, H^{(2)}\right)$. (The choice of the dielectric constant and solution at the interface will not change the error analysis result.) Then, Ampere's law at the material interface can be expressed as follows,

$$
-\left.\varepsilon_{2} \frac{\partial E_{y}^{(2)}}{\partial t}\right|_{x=x_{\mathrm{mat}}^{+}}=\left.\frac{\partial H_{z}^{(2)}}{\partial x}\right|_{x=x_{\mathrm{mat}}^{+}},
$$


where $x_{\text {mat }}^{+}=x_{\text {mat }}+\epsilon$ and $\epsilon$ is an infinitesimal positive number. The spatially discretized form of Eq. 2.40 in a regular Yee scheme is given by

$$
-\left.\varepsilon_{2} \frac{\partial E_{y}^{(2)}}{\partial t}\right|_{x_{\mathrm{mat}}^{+}}=\frac{H_{z}^{(2)}\left(x_{\mathrm{mat}}+h / 2\right)-H_{z}^{(1)}\left(x_{\mathrm{mat}}-h / 2\right)}{h}
$$

We define the error $\tau_{E_{y}}$ as the difference between the space derivative of $H_{z}$ with respect to $x$ and its corresponding discrete form, i.e.,

$$
\tau_{E_{y}}=\frac{H_{z}^{(2)}\left(x_{\mathrm{mat}}+h / 2\right)-H_{z}^{(1)}\left(x_{\mathrm{mat}}-h / 2\right)}{h}-\left.\frac{\partial H_{z}^{(2)}}{\partial x}\right|_{x=x_{\mathrm{mat}}^{+}} .
$$

Exploiting the Taylor series expansion method, we can expand Eq. 2.42 as follows,

$$
\begin{aligned}
\tau_{E_{y}}= & \frac{1}{h}\left(\left.H_{z}^{(2)}\right|_{x=x_{\mathrm{mat}}^{+}}-\left.H_{z}^{(1)}\right|_{x=x_{\mathrm{mat}}^{-}}\right)+\frac{1}{2}\left(\left.\frac{\partial H_{z}^{(1)}}{\partial x}\right|_{x=x_{\mathrm{mat}}^{-}}-\left.\frac{\partial H_{z}^{(2)}}{\partial x}\right|_{x=x_{\mathrm{mat}}^{+}}\right) \\
& +\frac{h}{8}\left(\left.\frac{\partial^{2} H_{z}^{(2)}}{\partial x^{2}}\right|_{x=x_{\mathrm{mat}}^{+}}-\left.\frac{\partial^{2} H_{z}^{(1)}}{\partial x^{2}}\right|_{x=x_{\mathrm{mat}}^{-}}\right)+O\left(h^{2}\right),
\end{aligned}
$$

where $x_{\text {mat }}^{-}$and $x_{\text {mat }}^{+}$refer to one-sided derivatives from the left and the right of the material interface, respectively. Consider the boundary conditions at the interface, we have the following equations,

$$
\begin{aligned}
& \left.E_{y}^{(1)}\right|_{x=x_{\mathrm{mat}}^{-}}=\left.E_{y}^{(2)}\right|_{x=x_{\mathrm{mat}}^{+}}, \\
& \left.H_{z}^{(1)}\right|_{x=x_{\mathrm{mat}}^{-}}=\left.H_{z}^{(2)}\right|_{x=x_{\mathrm{mat}}^{+}}
\end{aligned}
$$

Taking the first-order time derivative on both sides of Eq. 2.44a and combining it with Ampere's law $-\varepsilon \frac{\partial E_{y}}{\partial t}=\frac{\partial H_{z}}{\partial x}$, we obtain

$$
\left.\frac{1}{\varepsilon_{1}} \frac{\partial H_{z}^{(1)}}{\partial x}\right|_{x=x_{\mathrm{mat}}^{-}}=\left.\frac{1}{\varepsilon_{2}} \frac{\partial H_{z}^{(2)}}{\partial x}\right|_{x=x_{\mathrm{mat}}^{+}}
$$


Substituting Eqs. 2.44b and 2.45 into Eq. 2.43, yields

$$
\tau_{E_{y}}=\left.\frac{1}{2}\left(\frac{\varepsilon_{1}}{\varepsilon_{2}}-1\right) \frac{\partial H_{z}^{(2)}}{\partial x}\right|_{x=x_{\mathrm{mat}}^{+}}+O(h)
$$

One recovers a locally constant term as the leading local error, implying that the first-order global convergence can be expected [104].

If we assign an effective permittivity $\varepsilon=\left(\varepsilon_{1}+\varepsilon_{2}\right) / 2$ to the material interface, we can recover local first-order accuracy. Here, we define the error as

$$
\tau_{E_{y}}=\frac{2}{\varepsilon_{1}+\varepsilon_{2}} \frac{H_{z}^{(2)}\left(x_{\mathrm{mat}}+h / 2\right)-H_{z}^{(1)}\left(x_{\mathrm{mat}}-h / 2\right)}{h}-\left.\frac{1}{\varepsilon_{2}} \frac{\partial H_{z}^{(2)}}{\partial x}\right|_{x=x_{\mathrm{mat}}^{+}} .
$$

Using the Taylor series expansion method and combining with Eqs. 2.44b and 2.45, yields

$$
\tau_{E_{y}}=\frac{h}{4\left(\varepsilon_{1}+\varepsilon_{2}\right)}\left(\left.\frac{\partial^{2} H_{z}^{(2)}}{\partial x^{2}}\right|_{x=x_{\mathrm{mat}}^{+}}-\left.\frac{\partial^{2} H_{z}^{(1)}}{\partial x^{2}}\right|_{x=x_{\mathrm{mat}}^{-}}\right)+O\left(h^{2}\right)
$$

The local truncation error is of $O(h)$, which is sufficient to achieve global second-order convergence [104].

For the magnetic field $H_{z}$, we have Faraday's law as follows,

$$
\left.\mu_{0} \frac{\partial H_{z}^{(1)}}{\partial t}\right|_{x=x_{\mathrm{mat}}-h / 2}=\left.\frac{\partial E_{x}^{(1)}}{\partial y}\right|_{x=x_{\mathrm{mat}}-h / 2}-\left.\frac{\partial E_{y}^{(1)}}{\partial x}\right|_{x=x_{\mathrm{mat}}-h / 2}
$$

Consider the second term of R.H.S in Eq. 2.49 (the discrete space derivative of the first term does not cross the interface and then has the second-order accuracy), we can have the error as

$$
\tau_{H_{z}}=\frac{E_{y}^{(2)}\left(x_{\mathrm{mat}}\right)-E_{y}^{(1)}\left(x_{\mathrm{mat}}-h\right)}{h}-\left.\frac{\partial E_{y}^{(1)}}{\partial x}\right|_{x=x_{\mathrm{mat}}-h / 2}
$$

Taking the Taylor series expansion method for the last two terms at the R.H.S. with respect to $x=x_{\text {mat }}$, we can obtain

$$
\tau_{H_{z}}=\left.\frac{1}{24} \frac{\partial^{3} E_{y}^{(1)}}{\partial x^{3}}\right|_{x=x_{\mathrm{mat}}^{-}} h^{2}+O\left(h^{3}\right)
$$


The second-order accuracy for the magnetic field $H_{z}$ close to the material interface is achieved.

\subsubsection{TE case}

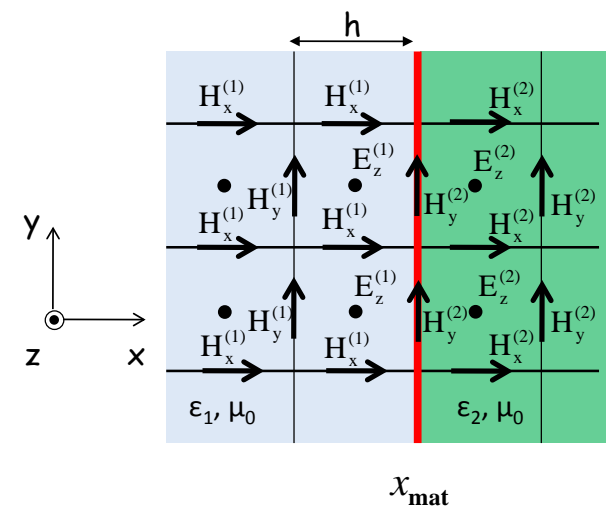

Figure 2.8 Illustration of Yee grids in the TE case. All others are the same as Figure 2.7.

The TE modes are defined as the modes with the field components $E_{z}, H_{x}$ and $H_{y}$ only. Similar to the TM case, we have two sets of fields $\left(E^{(k)}, H^{(k)}\right)(k=1,2)$ in the two different dielectric materials and have the interface aligned with the link of the TE Yee cell, as shown in Figure 2.8. At the interface, Faraday's law can be expressed as follows,

$$
\left.\mu_{0} \frac{\partial H_{y}^{(2)}}{\partial t}\right|_{x=x_{\mathrm{mat}}^{+}}=\left.\frac{\partial E_{z}^{(2)}}{\partial x}\right|_{x=x_{\mathrm{mat}}^{+}}
$$

And its spatially discretized form is

$$
\left.\mu_{0} \frac{\partial H_{y}^{(2)}}{\partial t}\right|_{x=x_{\mathrm{mat}}^{+}}=\frac{E_{z}^{(2)}\left(x_{\mathrm{mat}}+h / 2\right)-E_{z}^{(1)}\left(x_{\mathrm{mat}}-h / 2\right)}{h} .
$$

Analogous to the TM case, we define the error $\tau_{H_{y}}$ as

$$
\tau_{H_{y}}=\frac{E_{z}^{(2)}\left(x_{\mathrm{mat}}+h / 2\right)-E_{z}^{(1)}\left(x_{\mathrm{mat}}-h / 2\right)}{h}-\left.\frac{\partial E_{z}^{(2)}}{\partial x}\right|_{x=x_{\mathrm{mat}}^{+}} .
$$


Exploiting the Taylor series expansion method, Eq. 2.54 can be expressed as

$$
\begin{aligned}
\tau_{H_{y}}= & \frac{1}{h}\left(\left.E_{z}^{(2)}\right|_{x=x_{\mathrm{mat}}^{+}}-\left.E_{z}^{(1)}\right|_{x=x_{\mathrm{mat}}^{-}}\right)+\frac{1}{2}\left(\left.\frac{\partial E_{z}^{(1)}}{\partial x}\right|_{x=x_{\mathrm{mat}}^{-}}-\left.\frac{\partial E_{z}^{(2)}}{\partial x}\right|_{x=x_{\mathrm{mat}}^{+}}\right) \\
& +\frac{h}{8}\left(\left.\frac{\partial^{2} E_{z}^{(2)}}{\partial x^{2}}\right|_{x=x_{\mathrm{mat}}^{+}}-\left.\frac{\partial^{2} E_{z}^{(1)}}{\partial x^{2}}\right|_{x=x_{\mathrm{mat}}^{-}}\right)+O\left(h^{2}\right) .
\end{aligned}
$$

Taking the procedure similar to the TM case, we can use the boundary condition $\left.E_{z}^{(2)}\right|_{x=x_{\text {mat }}^{+}}=$ $\left.E_{z}^{(1)}\right|_{x=x_{\text {mat }}^{-}}$, and the equation $\left.\frac{\partial E_{z}^{(1)}}{\partial x}\right|_{x=x_{\text {mat }}^{-}}=\left.\frac{\partial E_{z}^{(2)}}{\partial x}\right|_{x=x_{\text {mat }}^{+}}$given by the boundary condition $\left.H_{y}^{(2)}\right|_{x=x_{\text {mat }}^{+}}=\left.H_{y}^{(1)}\right|_{x=x_{\text {mat }}^{-}}$and Faraday's law, to simplify Eq. 2.55, and yields

$$
\tau_{E_{y}}=\frac{h}{8}\left(\left.\frac{\partial^{2} E_{z}^{(2)}}{\partial x^{2}}\right|_{x=x_{\mathrm{mat}}^{+}}-\left.\frac{\partial^{2} E_{z}^{(1)}}{\partial x^{2}}\right|_{x=x_{\mathrm{mat}}^{-}}\right)+O\left(h^{2}\right) .
$$

Better than the TM case, the local first-order accuracy is achieved, implying the the global second-order convergence can be expected [104]. But if the interface is between two different magnetic materials, the first derivative of $E_{z}$ with respect to $x$ is not continuous across the interface, i.e., $\left.\frac{\partial E_{z}^{(1)}}{\partial x}\right|_{x=x_{\text {mat }}^{-}} \neq\left.\frac{\partial E_{z}^{(2)}}{\partial x}\right|_{x=x_{\text {mat }}^{+}}$. Instead, analogous to Eq. 2.45, we can have the following equation from Faraday's law,

$$
\left.\frac{1}{\mu_{1}} \frac{\partial E_{z}^{(1)}}{\partial x}\right|_{x=x_{\mathrm{mat}}^{-}}=\left.\frac{1}{\mu_{2}} \frac{\partial E_{z}^{(2)}}{\partial x}\right|_{x=x_{\mathrm{mat}}^{+}}
$$

Thus, similar to the TM case, we can obtain a locally constant term as the leading local error, and it can also be reduced to first order by assigning an effective permeability $\mu=\left(\mu_{1}+\mu_{2}\right) / 2$ to the interface.

Similar to $H_{z}$ in the TM case, $E_{z}$ in the TE case can also have the second-order accuracy,

$$
\tau_{E_{z}}=\left.\frac{1}{24} \frac{\partial^{3} H_{y}^{(1)}}{\partial x^{3}}\right|_{x=x_{\mathrm{mat}}^{-}} h^{2}+O\left(h^{3}\right)
$$




\subsection{The Total-field/Scattered-field Technique}

The total-field/scattered-field (TF/SF) formulation can realize a plane-wave source without the difficulties caused by using either hard sources or the initial-condition approach [87, $105,106]$. In this section, the $2 \mathrm{D}$ formulation of $\mathrm{TF} / \mathrm{SF}$ method is presented. In the real world, metamaterials are truncated and finite, but it is always interesting and useful to study an infinite metamaterial, which can provide sufficient information about the problem at hand. Most numerical researches in metamaterials only study the normal incidence case of an infinite-long metamaterial slab extended by the periodic boundary condition. In this case, the generation of the plane wave only needs 1D formulation, which is just a special case of the 2D formulation. By using the TF/SF method, the scattering amplitudes of the metamaterial structure are easy to obtain.

\subsubsection{Ideas}

The TF/SF formulation comes from the linearity of Maxwell's equations and the assumption that the physical total electric and magnetic field $\mathbf{E}_{\text {total }}$ and $\mathbf{H}_{\text {total }}$ can be decomposed in the following manner:

$$
\begin{gathered}
\mathbf{E}_{\text {total }}=\mathbf{E}_{\text {inc }}+\mathbf{E}_{\text {scatt }}, \\
\mathbf{H}_{\text {total }}=\mathbf{H}_{\text {inc }}+\mathbf{H}_{\text {scatt }},
\end{gathered}
$$

where $\mathbf{E}_{\mathrm{inc}}$ and $\mathbf{H}_{\mathrm{inc}}$ are the incident-wave fields and assumed to be known at all points of the computation space lattice at all time-steps [87]. $\mathbf{E}_{\text {scatt }}$ and $\mathbf{H}_{\text {scatt }}$ are the scattered-wave fields, which are initially unknown. For linear materials, the finite-difference operations of the Yee algorithm can be applied with equal validity to the incident field, the scattered field and the total field due to the linearity of Maxwell's equations [87]. This property allows zoning the Yee space lattice into two distinct regions [87] (see Figure 2.9): Region 1, where the total fields are assumed to be stored in the computer memory; Region 2 (surrounding Region 1), where instead of the total fields, the scattered fields are assumed to be stored. Region 1 and 
2 are separated by a nonphysical virtual surface which connects the fields in each region. The connecting condition generates the incident wave.

In Region 1, the Yee algorithm operates on the total fields, including the incident wave and the scattered wave. The structure we are interested in is embedded in this region.

In Region 2, the Yee algorithm operates on the scattered fields only. The outer boundary of Region 2 is terminated by the PML absorbing boundary condition, which absorbs the scattered fields and simulates the lattice extending to infinity.

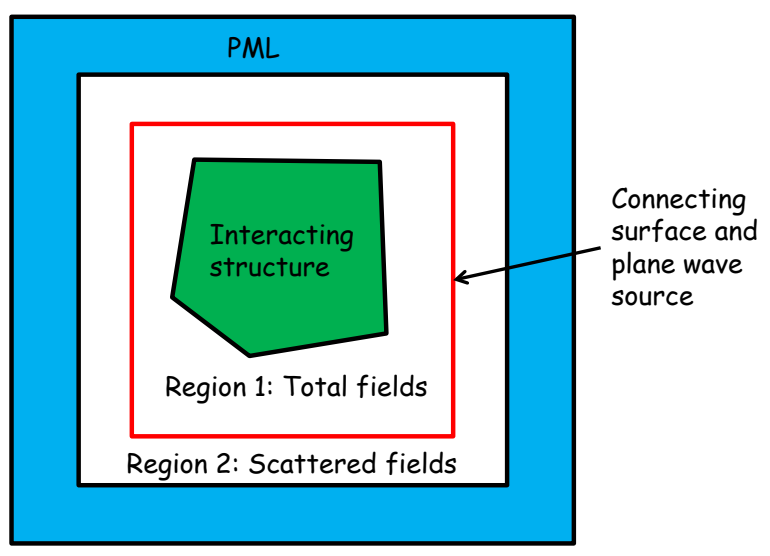

Figure 2.9 Illustration of the TF/SF zoning in the computation space domain.

\subsubsection{Two-dimensional formulation}

As we know, the FDTD spatial difference is operated on the total fields and the scattered fields in Region 1 and Region 2, respectively. When this operation is taken across the connecting surface, which constitutes the interface of these two regions, there arises an inconsistency problem since the need to use the fields in the neighboring Yee cell for the spatial difference enforces us to perform an arithmetic difference between the scattered- and total-field values. In this section, we present how to solve this inconsistency in the virtual connecting surface $[87,105,106]$.

a. TE case (only $H_{x}, H_{y}$ and $E_{z}$ present)

Using the Yee cell for the 2D TE case, which is shown in Figure 2.10, the update formula 
for the TE case in non-dispersive media are completely valid when all field components are the total-field components (or the scattered fields). But when we need to get the components on the interface, we have to take the spatial difference across the interface. Since the fields in Region 1 are assumed to be total fields, whereas the fields in Region 2 are assumed to be scattered fields, there arises a problem of consistency. For example, the update formulas for $E_{z}$ is as follows:

$$
\begin{aligned}
E_{z}^{l+1}(i, j)= & E_{z}^{l}(i, j)+\frac{\Delta t}{\varepsilon_{0} \Delta x}\left[H_{y}^{l+\frac{1}{2}}\left(i+\frac{1}{2}, j\right)-H_{y}^{l+\frac{1}{2}}\left(i-\frac{1}{2}, j\right)\right] \\
& -\frac{\Delta t}{\varepsilon_{0} \Delta y}\left[H_{x}^{l+\frac{1}{2}}\left(i, j+\frac{1}{2}\right)-H_{x}^{l+\frac{1}{2}}\left(i, j-\frac{1}{2}\right)\right]
\end{aligned}
$$

where we use the notation defined in Eq. A.1. When it is on the bottom interface where the total-field components $E_{z \text {, total }}$ and $H_{y}$, total are located (see Figure 2.10), from Eq. (2.60) we need to know $\left.H_{x, \text { total }}\right|_{i, j_{0}+1 / 2}$ and $\left.H_{x \text {, total }}\right|_{i, j_{0}-1 / 2}$ to time-step the $\left.E_{z \text {, total }}\right|_{i, j_{0}}$ components (indicated by circled dots in Figure 2.10). Clearly, the former $H_{x}$ is known since $\left(i, j_{0}+1 / 2\right)$ is in Region 1 where the total fields are stored. But $\left(i, j_{0}-1 / 2\right)$ is located in Region 2 where the scattered fields are stored, so only $\left.H_{x, \text { scatt }}\right|_{i, j_{0}-1 / 2}$ is available. Then from the values stored in memory, we will get

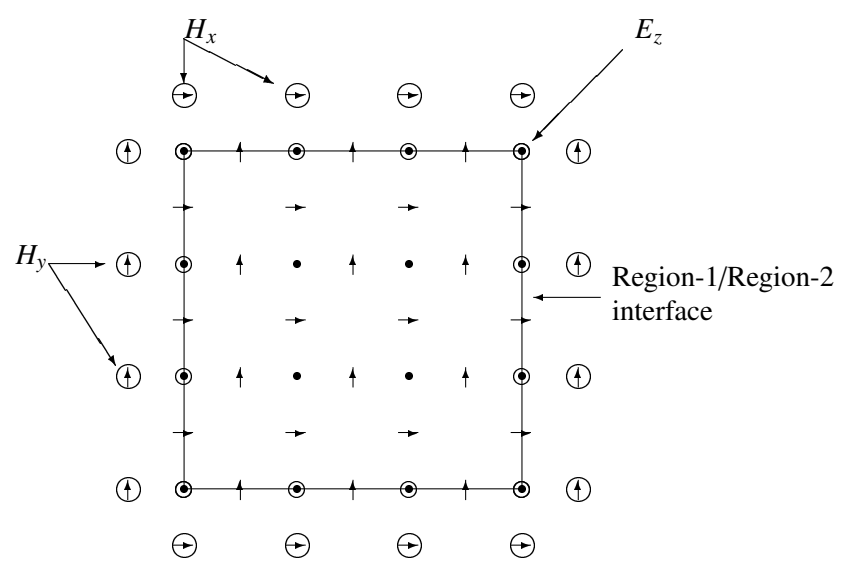

Figure 2.10 The Yee grid for the 2D TE case. The solid line indicates the interface between Region 1 (inside) and Region 2 (outside), which is aligned with $E_{z}$ components. The incident field data are required for $H_{x}$ and $H_{y}$ in the Yee cells surrounding the interface, and $E_{z}$ at the interface. 


$$
\begin{aligned}
E_{z, \text { total }}^{l+1}\left(i, j_{0}\right)= & E_{z, \text { total }}^{l}\left(i, j_{0}\right)+\frac{\Delta t}{\varepsilon_{0} \Delta x}\left[H_{y, \text { total }}^{l+\frac{1}{2}}\left(i+\frac{1}{2}, j_{0}\right)-H_{y, \text { total }}^{l+\frac{1}{2}}\left(i-\frac{1}{2}, j_{0}\right)\right] \\
& -\frac{\Delta t}{\varepsilon_{0} \Delta y}\left[H_{x, \text { total }}^{l+\frac{1}{2}}\left(i, j_{0}+\frac{1}{2}\right)-H_{x, \text { scatt }}^{l+\frac{1}{2}}\left(i, j_{0}-\frac{1}{2}\right)\right] .
\end{aligned}
$$

This is obviously inconsistent. To solve this problem, we use

$$
\mathbf{H}_{\text {total }}=\mathbf{H}_{\text {inc }}+\mathbf{H}_{\text {scatt }}
$$

in the valid update formulas. Then we have

$$
\begin{aligned}
E_{z, \text { total }}^{l+1}\left(i, j_{0}\right)= & E_{z, \text { total }}^{l}\left(i, j_{0}\right)+\frac{\Delta t}{\varepsilon_{0} \Delta x}\left[H_{y, \text { total }}^{l+\frac{1}{2}}\left(i+\frac{1}{2}, j_{0}\right)-H_{y, \text { total }}^{l+\frac{1}{2}}\left(i-\frac{1}{2}, j_{0}\right)\right] \\
& -\frac{\Delta t}{\varepsilon_{0} \Delta y}\left[H_{x, \text { total }}^{l+\frac{1}{2}}\left(i, j_{0}+\frac{1}{2}\right)-H_{x, \text { total }}^{l+\frac{1}{2}}\left(i, j_{0}-\frac{1}{2}\right)\right] \\
= & \underbrace{E_{z, \text { total }}^{l}\left(i, j_{0}\right)}_{\text {stored in memory }}+\frac{\Delta t}{\varepsilon_{0} \Delta x} \underbrace{\left[H_{y, \text { total }}^{l+\frac{1}{2}}\left(i+\frac{1}{2}, j_{0}\right)-H_{y, \text { total }}^{l+\frac{1}{2}}\left(i-\frac{1}{2}, j_{0}\right)\right]}_{\text {stored in memory }} \\
& -\frac{\Delta t}{\varepsilon_{0} \Delta y} \underbrace{\left[H_{x, \text { total }}^{l+\frac{1}{2}}\left(i, j_{0}+\frac{1}{2}\right)-H_{x, \text { scatt }}^{l+\frac{1}{2}}\left(i, j_{0}-\frac{1}{2}\right)\right]}_{\text {stored in memory }} \\
& +\frac{\Delta t}{\varepsilon_{0} \Delta y} \underbrace{H_{x, \text { inc }}^{l+\frac{1}{2}}\left(i, j_{0}-\frac{1}{2}\right)}_{\text {assumed known }} .
\end{aligned}
$$

For simplicity, we write it as

$$
E_{z}^{l+1}\left(i, j_{0}\right)=\left\{E_{z}^{l+1}\left(i, j_{0}\right)\right\}+\underbrace{\frac{\Delta t}{\varepsilon_{0} \Delta y} H_{x, \text { inc }}^{l+\frac{1}{2}}\left(i, j_{0}-\frac{1}{2}\right)}_{\text {assumed known correction term }}
$$

Here, the bracket denotes that the generic $E_{z}$ time-stepping operations of Eq. 2.60 can be done before adding the incident-wave correction term (we will use the bracket for the correction formula of other field components with the similar definition). After first applying the generic $E_{z}$ time-stepping at all $E_{z}$ locations in the grid, we can do this correction at the interface $E_{z}$ grid point. In this way, we do not need to break up the $E_{z}$ time-stepping loop.

The remaining $E_{z}$ components located on other Region-1 and Region-2 interfaces can be 
treated in an analogous manner to solve the inconsistency problem. The operations are as follows:

At the top interface between Region 1 and Region $2\left(j=j_{1}, i=i_{0}, \cdots, i_{1}\right)$,

$$
E_{z}^{l+1}\left(i, j_{1}\right)=\left\{E_{z}^{l+1}\left(i, j_{1}\right)\right\}-\underbrace{\frac{\Delta t}{\varepsilon_{0} \Delta y} H_{x, \text { inc }}^{l+\frac{1}{2}}\left(i, j_{1}+\frac{1}{2}\right)} .
$$

assumed known correction term

At the left interface $\left(i=i_{0}, j=j_{0}, \cdots, j_{1}\right)$,

$$
E_{z}^{l+1}\left(i_{0}, j\right)=\left\{E_{z}^{l+1}\left(i_{0}, j\right)\right\}-\underbrace{\frac{\Delta t}{\varepsilon_{0} \Delta x} H_{y, \text { inc }}^{l+\frac{1}{2}}\left(i_{0}-1 / 2, j\right)}_{\text {assumed known correction term }}
$$

At the right interface $\left(i=i_{1}, j=j_{0}, \cdots, j_{1}\right)$,

$$
E_{z}^{l+1}\left(i_{1}, j\right)=\left\{E_{z}^{l+1}\left(i_{1}, j\right)\right\}+\underbrace{\frac{\Delta t}{\varepsilon_{0} \Delta x} H_{y, \text { inc }}^{l+\frac{1}{2}}\left(i_{1}+1 / 2, j\right)}_{\text {assumed known correction term }}
$$

For the $H_{x}$ and $H_{y}$ components, we can get the following equations using the analogous manner with $E_{z}$ :

Outside the bottom interface of Region $1\left(j=j_{0}-1 / 2, i=i_{0}, \cdots, i_{1}\right)$

$$
H_{x}^{l+1 / 2}\left(i, j_{0}-1 / 2\right)=\left\{H_{x}^{l+1 / 2}\left(i, j_{0}-1 / 2\right)\right\}+\frac{\Delta t}{\mu_{0} \Delta y} E_{z, \text { inc }}^{l}\left(i, j_{0}\right)
$$

Outside the top interface of Region $1\left(j=j_{1}+1 / 2, i=i_{0}, \cdots, i_{1}\right)$

$$
H_{x}^{l+1 / 2}\left(i, j_{1}+1 / 2\right)=\left\{H_{x}^{l+1 / 2}\left(i, j_{1}+1 / 2\right)\right\}-\frac{\Delta t}{\mu_{0} \Delta y} E_{z, \text { inc }}^{l}\left(i, j_{1}\right)
$$

Outside the left interface of Region $1\left(i=i_{0}-1 / 2, j=j_{0}, \cdots, j_{1}\right)$

$$
H_{y}^{l+1 / 2}\left(i_{0}-1 / 2, j\right)=\left\{H_{y}^{l+1 / 2}\left(i_{0}-1 / 2, j\right)\right\}-\frac{\Delta t}{\mu_{0} \Delta x} E_{z, \text { inc }}^{l}\left(i_{0}, j\right) .
$$


Outside the right interface of Region $1\left(i=i_{1}+1 / 2, j=j_{0}, \cdots, j_{1}\right)$

$$
H_{y}^{l+1 / 2}\left(i_{1}+1 / 2, j\right)=\left\{H_{y}^{l+1 / 2}\left(i_{1}+1 / 2, j\right)\right\}+\frac{\Delta t}{\mu_{0} \Delta x} E_{z, \text { inc }}^{l}\left(i_{1}, j\right) .
$$

b. TM case

Taking the Yee grid and the connecting surface as Figure 2.11 and using the manner analogous to the 2D TE case, we can have the correction formula:

At the bottom interface of Region $1\left(j=j_{0}+1 / 2, i=i_{0}+1 / 2, \cdots, i=i_{1}-1 / 2\right)$

$$
H_{z}^{l+1 / 2}\left(i+1 / 2, j_{0}+1 / 2\right)=\left\{H_{z}^{l+1 / 2}\left(i+1 / 2, j_{0}+1 / 2\right)\right\}-\underbrace{\frac{\Delta t}{\mu_{0} \Delta y} E_{x, \text { inc }}^{l}\left(i+1 / 2, j_{0}\right)} .
$$

assumed known correction term

At the top interface of Region $1\left(j=j_{1}-1 / 2, i=i_{0}+1 / 2, \cdots, i=i_{1}-1 / 2\right)$

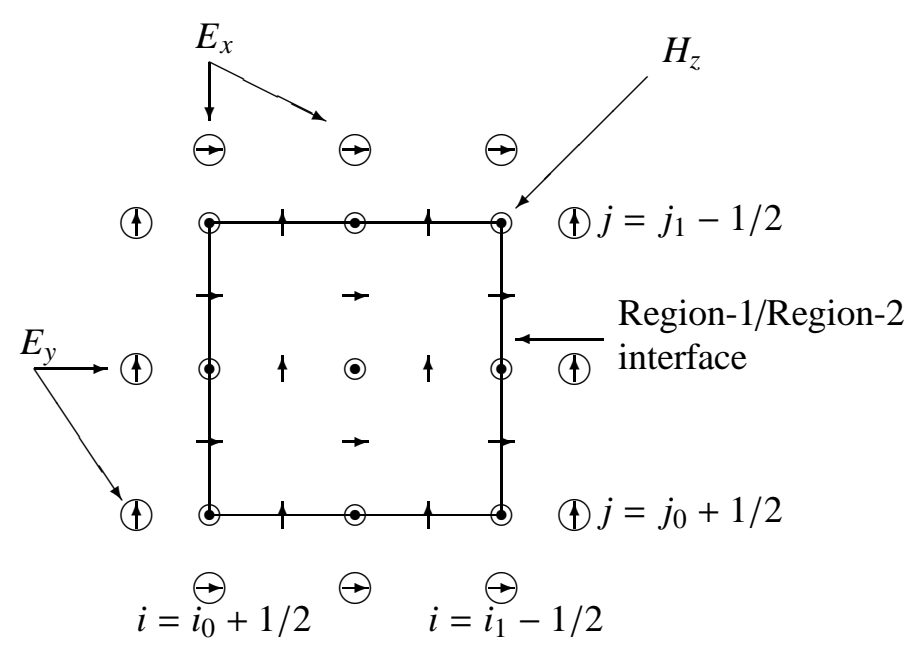

Figure 2.11 The Yee grid for the 2D TM case. The solid line indicates the interface between Region 1 (inside) and Region 2 (outside), which is aligned with the magnetic field components. The incident field data are required for $H_{z}$ at the interface, and $E_{x}$ and $E_{y}$ in the Yee cells surrounding the interface.

$$
H_{z}^{l+1 / 2}\left(i+1 / 2, j_{1}-1 / 2\right)=\left\{H_{z}^{l+1 / 2}\left(i+1 / 2, j_{1}-1 / 2\right)\right\}+\underbrace{\frac{\Delta t}{\mu_{0} \Delta y} E_{x, \text { inc }}^{l}\left(i+1 / 2, j_{1}\right)} .
$$


At the left interface of Region $1\left(i=i_{0}+1 / 2, j=j_{0}+1 / 2, \cdots, j=j_{1}-1 / 2\right)$

$$
H_{z}^{l+1 / 2}\left(i_{0}+1 / 2, j+1 / 2\right)=\left\{H_{z}^{l+1 / 2}\left(i_{0}+1 / 2, j+1 / 2\right)\right\}+\underbrace{\frac{\Delta t}{\mu_{0} \Delta x} E_{y, \text { inc }}^{l}\left(i_{0}, j+1 / 2\right)}_{\text {assumed known correction term }} .
$$

At the right interface of Region $1\left(i=i_{1}-1 / 2, j=j_{0}+1 / 2, \cdots, j=j_{1}-1 / 2\right)$

$$
H_{z}^{l+1 / 2}\left(i_{1}-1 / 2, j+1 / 2\right)=\left\{H_{z}^{l+1 / 2}\left(i_{1}-1 / 2, j+1 / 2\right)\right\}-\underbrace{\frac{\Delta t}{\mu_{0} \Delta x} E_{y, \text { inc }}^{l}\left(i_{1}, j+1 / 2\right)}_{\text {assumed known correction term }} .
$$

For the $E_{x}$ and $E_{y}$ components, we have the following correction formula:

Outside the bottom interface of Region $1\left(j=j_{0}, i=i_{0}+1 / 2, \cdots, i_{1}-1 / 2\right)$

$$
E_{x}^{l+1}\left(i+1 / 2, j_{0}\right)=\left\{E_{x}^{l+1}\left(i+1 / 2, j_{0}\right)\right\}-\frac{\Delta t}{\varepsilon_{0} \Delta y} H_{z, \text { inc }}^{l+1 / 2}\left(i+1 / 2, j_{0}+1 / 2\right) .
$$

Outside the top interface of Region $1\left(j=j_{1}, i=i_{0}+1 / 2, \cdots, i_{1}-1 / 2\right)$

$$
E_{x}^{l+1}\left(i+1 / 2, j_{1}\right)=\left\{E_{x}^{l+1}\left(i+1 / 2, j_{1}\right)\right\}+\frac{\Delta t}{\varepsilon_{0} \Delta y} H_{z, \text { inc }}^{l+1 / 2}\left(i+1 / 2, j_{1}-1 / 2\right) .
$$

Outside the left interface of Region $1\left(i=i_{0}, j=j_{0}+1 / 2, \cdots, j_{1}-1 / 2\right)$

$$
E_{y}^{l+1}\left(i_{0}, j+1 / 2\right)=\left\{E_{y}^{l+1}\left(i_{0}, j+1 / 2\right)\right\}+\frac{\Delta t}{\varepsilon_{0} \Delta x} H_{z, \text { inc }}^{l+1 / 2}\left(i_{0}+1 / 2, j+1 / 2\right) .
$$

Outside the right interface of Region $1\left(i=i_{1}, j=j_{0}+1 / 2, \cdots, j_{1}-1 / 2\right)$

$$
E_{y}^{l+1}\left(i_{1}, j+1 / 2\right)=\left\{E_{y}^{l+1}\left(i_{1}, j+1 / 2\right)\right\}-\frac{\Delta t}{\varepsilon_{0} \Delta x} H_{z, \text { inc }}^{l+1 / 2}\left(i_{1}-1 / 2, j+1 / 2\right) .
$$

\subsubsection{Calculation of the incident field}

Assume the plane wave is propagating with a wavevector $\mathbf{k}_{\mathrm{inc}}$ that is oriented at the angle $\phi$ relative to the $+x$-axis of the FDTD grid. Referring to Figure 2.12, we can get the first grid 


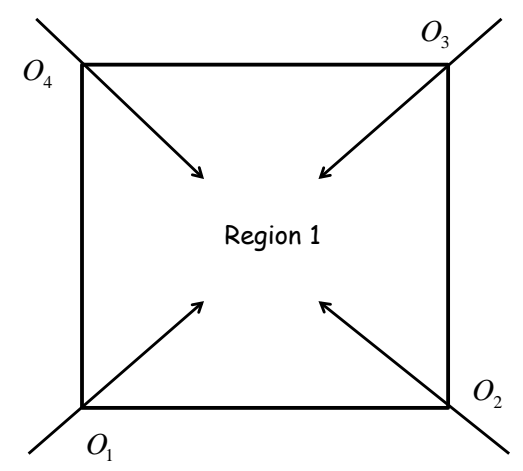

Figure 2.12 Illustration of the incident wave impinged on Region 1 from four different directions. In each direction, Region 1 has a different first contacted grid point by the incident wavefront.

point in Region 1 (it will be set as origin) contacted by the incident wavefront for various $\phi$ :

$$
\begin{array}{cc}
O_{1}: & 0^{0}<\phi \leq 90^{0} \\
O_{2}: & 90^{0}<\phi \leq 180^{0} \\
O_{3}: & 180^{0}<\phi \leq 270^{0} \\
O_{4}: & 270^{0}<\phi \leq 360^{0}
\end{array}
$$

Assume $\mathbf{r}_{\text {comp }}$ is the position vector from the origin $O$ to the location of the field vector component of interest, it can be given by

$$
\mathbf{r}_{\mathrm{comp}}=\left(i_{\mathrm{comp}}-i_{o}\right) \hat{x}+\left(j_{\mathrm{comp}}-j_{o}\right) \hat{y}
$$

It is clear that $\mathbf{E}_{\text {inc }}$ and $\mathbf{H}_{\text {inc }}$ can be calculated at any grid location using an analytical expression for the space-time behavior of the incident wave if its position vector is provided. But it will need a large amount of computer arithmetic and be pretty time-consuming. To reduce the burden, we use an approach based upon a table look-up procedure [87].

We assume that an auxiliary one-dimensional source FDTD grid is placed along the incident wavevector so that origin $O$ of Region 1 which we get from Eq. 2.80 coincides with $E_{\text {inc }}\left(m_{0}\right)$ (TE case) or $H_{\text {inc }}\left(m_{0}+1 / 2\right)$ (TM case) $\left(m_{0}\right.$ is the space-step number in the source grid), one of the $E / H$-field components of the source grid. The idea is to use the linear source grid with 
the same space step $\Delta$, time step $\Delta t$ and time-step number $l$ as the main computation lattice, to calculate the incident fields in free space [87]. In this manner, the source grid generates a look-up table for the incident fields. With the delay distance $d\left(d=\hat{k}_{\text {inc }} \cdot \mathbf{r}_{\text {comp }}\right.$, where $\hat{k}_{\text {inc }}$ is the unit wavevector) for a point in the main space lattice, the incident fields at this point can be obtained by interpolation [87].

This procedure requires computation of the incident-wave time dependence at only a single point on the source grid [87], the hard source at $m_{0}-2$ :

$$
E_{\text {inc }}^{l}\left(m_{0}-2\right)=E_{0} g(l \Delta t)
$$

where $g$ is an arbitrary time function.

The FDTD update algorithms for the linear source grid are as follows:

$$
\begin{gathered}
E_{\mathrm{inc}}^{l+1}(m)=E_{\mathrm{inc}}^{l}(m)+\frac{\Delta t}{\left[\frac{\tilde{v}_{p}\left(\phi=0^{0}\right)}{\tilde{v}_{p}(\phi)}\right] \varepsilon_{0} \Delta}\left(H_{\mathrm{inc}}^{l+1 / 2}(m-1 / 2)-H_{\mathrm{inc}}^{l+1 / 2}(m+1 / 2)\right), \\
H_{\mathrm{inc}}^{l+1 / 2}(m+1 / 2)=H_{\mathrm{inc}}^{l-1 / 2}(m+1 / 2)+\frac{\Delta t}{\left[\frac{\tilde{v}_{p}\left(\phi=0^{0}\right)}{\tilde{v}_{p}(\phi)}\right] \mu_{0} \Delta}\left(E_{\mathrm{inc}}^{l}(m)-E_{\mathrm{inc}}^{l}(m+1)\right),
\end{gathered}
$$

where the factor $\tilde{v}_{p}\left(\phi=0^{0}\right) / \tilde{v}_{p}(\phi)$ is the ratio of numerical phase velocities in the TE and TM grids and slightly less than 1, hence, with the introduction of this factor as a multiplier of both $\mu_{0}$ and $\varepsilon_{0}$, the numerical wave propagation in the grid gets slightly faster. To get $\tilde{v}_{p}\left(\phi=0^{0}\right) / \tilde{v}_{p}(\phi)$, here we have $\tilde{v}_{p}\left(\phi=0^{0}\right)[87]$

$$
\tilde{v}_{p}\left(\phi=0^{0}\right)=\frac{\omega}{\tilde{k}}=\frac{\pi}{N_{\lambda} \sin ^{-1}\left[\frac{1}{S} \sin \left(\frac{\pi S}{N_{\lambda}}\right)\right]} c
$$

where $S=c \Delta t / \Delta$ and $N_{\lambda}=\lambda_{0} / \Delta$ (suppose $\left.\Delta x=\Delta y=\Delta\right)$. $\tilde{v}_{p}(\phi)$ can be obtained from the following numerical dispersion relation for two dimensional wave propagation:

$$
\frac{1}{S^{2}} \sin ^{2}\left(\frac{\pi S}{N_{\lambda}}\right)=\sin ^{2}\left(\frac{\Delta \cdot \tilde{k} \cos \phi}{2}\right)+\sin ^{2}\left(\frac{\Delta \cdot \tilde{k} \sin \phi}{2}\right) .
$$


For any $\phi$, the wavevector $\tilde{k}$ can be obtained by applying the Newton's method iterative procedure to Eq. 2.86 [87]:

$$
\tilde{k}_{\text {icount }+1}=\tilde{k}_{\text {icount }}-\frac{\sin ^{2}\left(A \tilde{k}_{\text {icount }}\right)+\sin ^{2}\left(B \tilde{k}_{\text {icount }}\right)-C}{A \sin \left(2 A \tilde{k}_{\text {icount }}\right)+B \sin \left(2 B \tilde{k}_{\text {icount }}\right)},
$$

where $A, B$, and $C$ are coefficients given by

$$
A=\frac{\Delta \cdot \cos \phi}{2}, \quad B=\frac{\Delta \cdot \sin \phi}{2}, \quad C=\frac{1}{S^{2}} \sin ^{2}\left(\frac{\pi S}{N_{\lambda}}\right)
$$

Then $\tilde{v}_{p}$ can be given by

$$
\frac{\tilde{v}_{p}}{c}=\frac{2 \pi}{\tilde{k}_{\text {finalicount }} \lambda_{0}}
$$

To obtain the incident fields at any given point in the main computation lattice, we will linearly interpolate the field values in the source grid [87]. For convenience, we use IFIX(r) to denote the largest integer in the real number $\mathrm{r}$.

For TE case, we have the interpolation equations for an E-field located at position $\mathbf{r}_{\text {comp }}$ with the delay distance $d$ :

$$
\begin{aligned}
d^{\prime} & =d-\operatorname{IFIX}(d), \\
E_{\mathrm{inc}}^{l}(d) & =\left(1-d^{\prime}\right) \cdot E_{\mathrm{inc}}^{l}\left(m_{0}+\operatorname{IFIX}(d)\right)+d^{\prime} \cdot E_{\mathrm{inc}}^{l}\left(m_{0}+\operatorname{IFIX}(d)+1\right) .
\end{aligned}
$$

For a magnetic field located at $d$, we have similar equations

$$
\begin{aligned}
d^{\prime \prime}= & d+1 / 2, \\
d^{\prime}= & d^{\prime \prime}-\operatorname{IFIX}\left(d^{\prime \prime}\right), \\
H_{\mathrm{inc}}^{l+1 / 2}(d)= & \left(1-d^{\prime}\right) \cdot H_{\mathrm{inc}}^{l+1 / 2}\left(m_{0}-1 / 2+\operatorname{IFIX}\left(d^{\prime \prime}\right)\right) \\
& +d^{\prime} \cdot H_{\mathrm{inc}}^{l+1 / 2}\left(m_{0}+1 / 2+\operatorname{IFIX}\left(d^{\prime \prime}\right)\right) .
\end{aligned}
$$

With the above interpolation equations, we can obtain the incident field components used to 
implement the correction formula,

$$
\begin{aligned}
E_{z, \text { inc }}^{l}(d) & =E_{i n c}^{l}(d), \\
H_{x, \text { inc }}^{l+1 / 2}(d) & =H_{\mathrm{inc}}^{l+1 / 2} \sin \phi, \\
H_{y, \text { inc }}^{l+1 / 2}(d) & =-H_{\mathrm{inc}}^{l+1 / 2} \cos \phi .
\end{aligned}
$$

For TM case, we can obtain the interpolation equations for a magnetic field located at the delay distance $d$ :

$$
\begin{aligned}
d^{\prime}= & d-\operatorname{IFIX}(d), \\
H_{\mathrm{inc}}^{l+1 / 2}(d)= & \left(1-d^{\prime}\right) H_{\mathrm{inc}}^{l+1 / 2}\left(m_{0}+\operatorname{IFIX}(d)+1 / 2\right) \\
& +d^{\prime} H_{\mathrm{inc}}^{l+1 / 2}\left(m_{0}+\operatorname{IFIX}(d)+3 / 2\right) .
\end{aligned}
$$

For the electric field located at the delay distance $d$, we have

$$
\begin{aligned}
d^{\prime \prime}= & d+1 / 2, \\
d^{\prime}= & d^{\prime \prime}-\operatorname{IFIX}\left(d^{\prime \prime}\right), \\
E_{\mathrm{inc}}^{l}(d)= & \left(1-d^{\prime}\right) E_{\mathrm{inc}}^{l}\left(m_{0}+\operatorname{IFIX}\left(d^{\prime \prime}\right)\right) \\
& +d^{\prime} \cdot E_{\mathrm{inc}}^{l}\left(m_{0}+\operatorname{IFIX}\left(d^{\prime \prime}\right)+1\right) .
\end{aligned}
$$

The incident field components used to implement the correction formula are

$$
\begin{aligned}
H_{z, \text { inc }}^{l+1 / 2}(d) & =H_{\mathrm{inc}}^{l+1 / 2}(d), \\
E_{x, \text { inc }}^{l}(d) & =-E_{\mathrm{inc}}^{l} \sin \phi, \\
E_{y, \text { inc }}^{l}(d) & =E_{\mathrm{inc}}^{l} \cos \phi .
\end{aligned}
$$




\section{CHAPTER 3. OPTICAL ANISOTROPIC METAMATERIALS: NEGATIVE REFRACTION AND FOCUSING}

\subsection{Introduction}

Recently, negative index materials (NIMs) and photonic crystals (PCs) are receiving more and more attention because of their extraordinary optical properties such as near field focusing, subwavelength imaging, and negative refraction [1-3, 107-115]. As first proposed, these NIMs have the permittivity, $\varepsilon$, and the permeability, $\mu$, simultaneously negative, which are achieved by overlapping electric and magnetic resonances. But the double resonance scheme also causes large resonance losses and technical difficulties in design and fabrication. In addition to negative index materials, both theoretical and experimental studies show the properties of negative refraction and subwavelength imaging can also occur in some uniaxially anisotropic media, which can have lower losses and be easier to fabricate [51-61].

For a particular anisotropic medium, where the permittivity component $\left(\varepsilon_{\perp}\right)$ along the direction perpendicular to the interface is negative, while all other permittivity and permeability components are positive, it has a hyperbolic dispersion relation as follows:

$$
\frac{k_{\perp}^{2}}{\left|\varepsilon_{\|}\right| \mu}-\frac{k_{\|}^{2}}{\left|\varepsilon_{\perp}\right| \mu}=\omega^{2},
$$

where the definitions for $\varepsilon_{\perp}, \varepsilon_{\|}, k_{\perp}$ and $k_{\|}$are shown in Figure 3.1(b). Figure 3.1(a) schematically shows how negative refraction works in this particular anisotropic medium. The group velocity can be calculated by $\mathbf{v}_{g}=\nabla_{\mathbf{k}} \omega(\mathbf{k})$, which implies that the direction of group velocity (energy flow) would be normal to the equifrequency surface (EFS) and in the direction where $\omega$ is increasing. The conservation of $k_{\|}$indicates two possible solutions in the medium, but the 


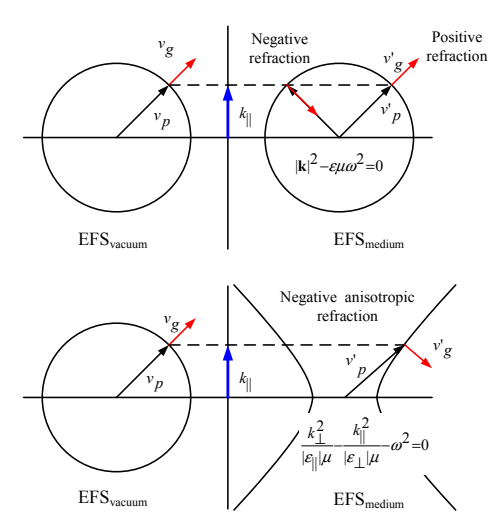

(a)

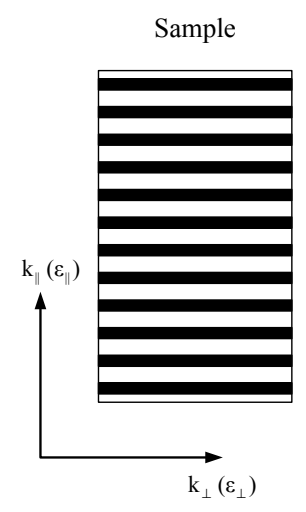

(b)

Figure 3.1 (a) Top graph: Circular equifrequency surfaces (EFS) for vacuum and isotropic media. Bottom graph: Equifrequency surfaces for vacuum (circle) and negative anisotropic refraction media (hyperbolic relation). (b) The definitions for $k_{\perp}, k_{\|}, \varepsilon_{\perp}$, and $\varepsilon_{\|}$used in our simulations.

correct one can be determined by causality - the refracted group velocity should point away from the interface, as shown in Figure 3.1(a). From Figure 3.1(a), we can also see that for an isotropic medium, the circular equifrequency surface forces the refracted phase and group velocities to lie in the same line - antiparallel for a negative index medium, while parallel for a positive index medium. For an anisotropic medium with a hyperbolic dispersion relation, they do not lie in the same line any more except for the case when $k_{\|}=0$. To be normal to the hyperbolic curves and satisfy the requirement of pointing away from the interface coming from the causality, the refracted group velocity has to undergo a negative refraction, which causes the expected focusing. (Note that the refracted phase velocity for an anisotropic medium still has a positive refraction.)

A lot of work has been done in anisotropic metamaterials, both experimentally $[53,56]$ and theoretically [51, 52, 54, 55, 57-61]. Liu and Zhang [61] derived the hyperbolic dispersion only theoretically in the Maxwell-Garnett approximation. Although they showed negative refraction and pseudo focusing in numerical simulations, they did not obtain the actual dispersion relation from the realistic simulated metamaterial nor did they demonstrate the effective medium behavior from realistic simulations. There is a need to demonstrate that the hyper- 
bolic dispersion survives all the way up to the evanescent waves, which is essential for potential super-resolution. Silveirinha et al.[54], apart from analytical calculations, also did not demonstrate the hyperbolic dispersion of the simulated metamaterial. They only showed the near-field imaging (channeling), which occurs for the special case of a very flat dispersion. Yao et al. [56] did experimental work (negative refraction for small angles only and no dispersion relation was obtained from the experiments), and Wangberg et al. [55] presented analytical work based on the Maxwell-Garnett approximation. Most of the previous theoretical and numerical work on anisotropic metamaterials is done on homogeneous materials, where the hyperbolic dispersion relation given by Eq. 3.1 is used.

In this chapter, we use realistic simulations for three-dimensional (3D) wire media and metal-dielectric superlattices to establish directly that the hyperbolic dispersion relation is valid up to evanescent modes in the long-wavelength limit and then retrieve the effective permittivity. A fitting procedure is exploited to get the dispersion relation from the field distributions obtained from full-wave numerical simulations of realistic structures. The imaging for a homogeneous slab with the effective permittivity shows very good agreement with the realistic structure. (All simulations about this homogeneous effective anisotropic medium are done by comsol multiphysics, an electromagnetic (EM) solver based on the finite element method.) We have three significant contributions to the field of anisotropic metamaterials: (1) the numerically obtained dispersion relations, (2) the demonstration of the effective medium behavior that works with evanescent incident modes and (3) our unique method to obtain the dispersion relations, different from the usual retrieval procedure based on inverting the scattering amplitudes.

\subsection{Superlattice of Metallic-air Layers}

Before discussing our results on 3D wire media, simulations are performed for a superlattice of metallic layers with $\varepsilon=-4$ and air layers with $\varepsilon=1$ as shown in Figure 3.1(b). These simulations are done to check the applicability of our idea that one can obtain negative refraction and focusing in anisotropic media. Our simulation results show the existence of negative 


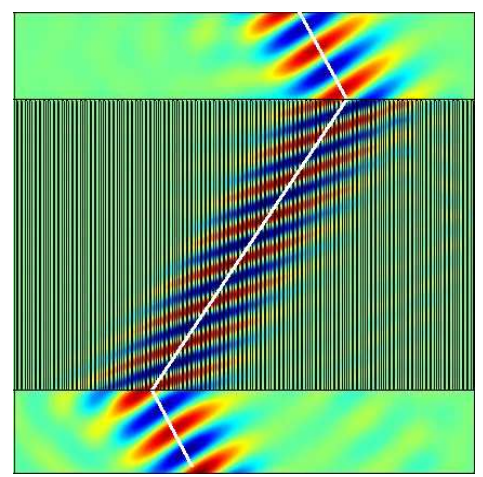

(a)

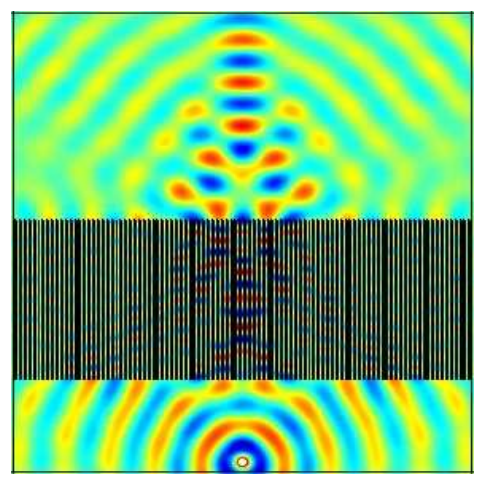

(c)

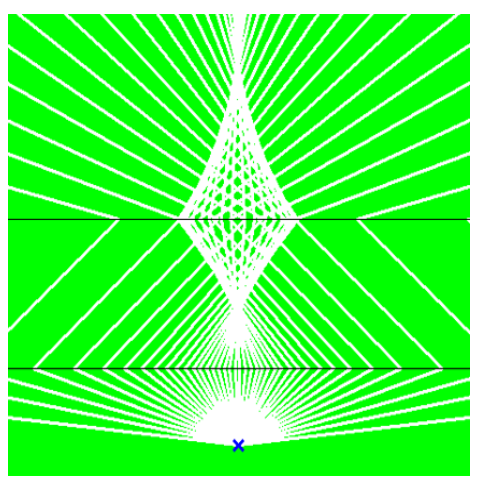

(b)

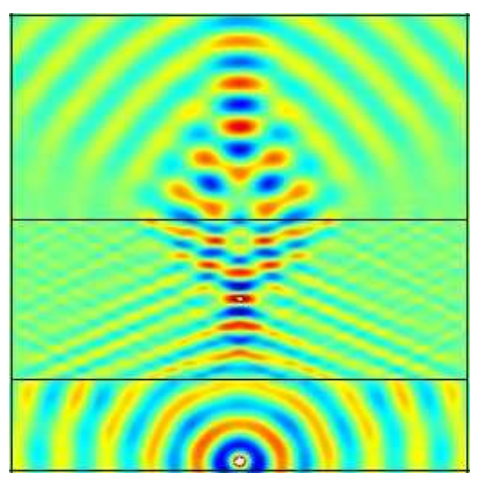

(d)

Figure 3.2 Material parameters of the metallic layers: $\varepsilon=-4, \mu=1$; working frequency $f=0.5 \mathrm{GHz}$; space period of metallic layers is $0.06 \mathrm{~m}$; width of metallic layers is $0.02 \mathrm{~m}$. (a) The magnetic field distribution of the group negative refraction in the metallic-air layers array slab for a transverse magnetic Gaussian beam with an incident angle of $30^{\circ}$. The white line indicates the ray-tracing result. (b) A ray-tracing diagram showing that the rays coming from a line source are refocused by an anisotropic medium slab with the effective permittivity $\varepsilon$ tensor of our simulated metallic-air layers array slab. (c) The magnetic field distribution of the pseudo focusing of our simulated metallicplates array slab with a line source placed $1.25 \mathrm{~m}$ from the interface, which launches a cylindrical transverse magnetic polarized wave. The thickness and the width of the metallic-air layers array slab are $2.4 \mathrm{~m}$ and $6 \mathrm{~m}$, respectively. (d) The magnetic field distribution of the pseudo focusing in a homogeneous anisotropic slab with the effective permittivity of our simulated metallic-air layers array slab $\left(\varepsilon_{\|}=1.7293, \varepsilon_{\perp}=-0.7907\right)$. 
refraction in Figure 3.2(a) and pseudo-focusing in Figure 3.2(c). The focusing simulation is compared with the ray-tracing diagram (Figure 3.2(b)) and the imaging of a homogeneous anisotropic slab with the effective permittivity extracted from the dispersion relation of the metallic-air superlattice (Figure 3.2(d)). The effective parameters for $\varepsilon_{\|}$and $\varepsilon_{\perp}$ are obtained by extracting $\mathbf{k}$ from the field distribution of a plane wave incidence inside the slab and then fitting with the hyperbolic dispersion given by Eq. 3.1. The details for obtaining the effective parameters $\varepsilon_{\|}$and $\varepsilon_{\perp}$ will be discussed below. One can see the pseudo focusing for the real metallic-air superlattice agrees very well with the homogeneous medium.

\subsection{Obtained Numerical Dispersion Relations}

To check if the wire medium constitutes our desired homogeneous effective anisotropic medium, it is straightforward to obtain its numerical dispersion relation first. For this purpose, we exploit a fitting procedure to extract $\mathbf{k}$ from the phase propagation. In the long wavelength limit, electromagnetic metamaterials should behave like a homogeneous medium. When a plane wave incidents on a homogeneous slab with an incident angle $\theta_{i}$, it forms a stationary wave inside the slab instead of a traveling wave because of the reflections at the two interfaces. Since $k_{\perp}$ represents the field variation in the perpendicular direction, we can take a crosssection along this direction and analytically obtain the field distribution in the cross-section by considering the multireflections inside the slab as follows:

$$
\begin{aligned}
F(y)= & \frac{A}{1-r^{2} e^{-2 \alpha d} e^{2 i k_{\perp} d}}\left[e^{-\alpha\left(y-y_{0}\right)} e^{i\left[k_{\perp}\left(y-y_{0}\right)+\theta\right]}\right. \\
& \left.+r e^{\alpha\left(y-y_{0}-2 d\right)} e^{-i\left[k_{\perp}\left(y-y_{0}-2 d\right)-\theta\right]}\right] .
\end{aligned}
$$

Here $y$ is the position in the perpendicular direction within the cross section, $F(y)$ is the field at the position $y, A$ and $\theta$ are the field amplitude and the field phase, respectively, at the starting point of the cross-section in the perpendicular direction $y=y_{0}$ (i.e., the location of the first interface of the slab), $\alpha$ is the decay factor of the homogeneous slab, $k_{\perp}$ is the perpendicular component of the wave vector $\mathbf{k}, d$ is the thickness of the slab, and $r$ is the reflection coefficient 
at the two interfaces.

By fitting the numerically obtained field distribution along the perpendicular direction in a cross-section with the theoretical formulas above, we can obtain the $k_{\perp}$ inside the wire medium slab for an incident plane wave with an incident angle, $\theta_{i}$. For $k_{\|}$, we can easily get $k_{\|}=k_{0} \sin \theta_{i}$ from the incident angle $\theta_{i}$, since $k_{\|}$is conserved across the interfaces, where $k_{0}$ is the wavevector in the background. Consequently, we can have the numerical dispersion relation of the wire medium by obtaining $k_{\|}$and $k_{\perp}$ for different incident angles.

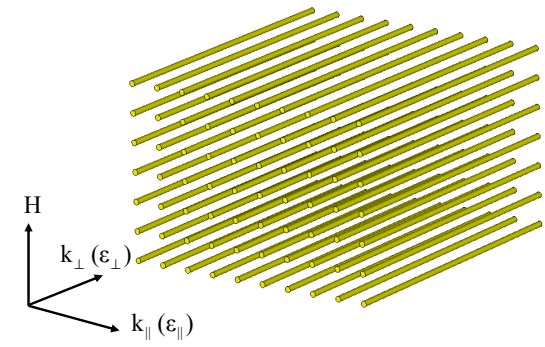

(a)

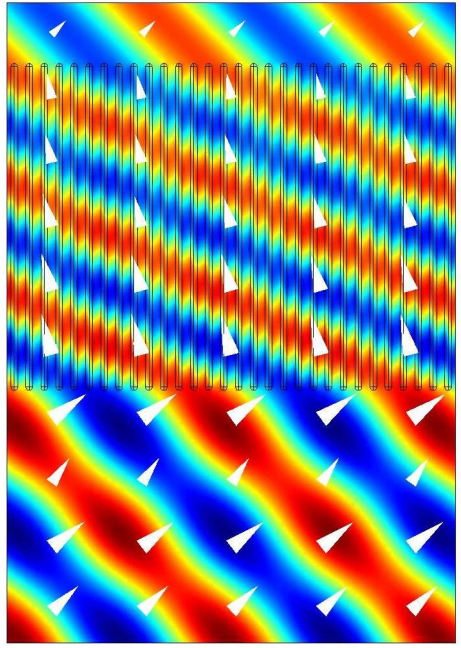

(b)

Figure 3.3 (a) Schematic of 3D metallic wires embedded in a dielectric matrix. (b) The magnetic field distribution for the negative refraction in a 3D gold-wire square-lattice medium with vacuum background and the wavelength $\lambda=700 \mathrm{~nm}$. The incident plane wave has transverse magnetic polarization and an incident angle of $45^{\circ}$. The permittivity $\varepsilon$ for gold is taken from experimental data [116]: $\varepsilon=-15.5931+i 1.2734$ at $\lambda=700 \mathrm{~nm}$. The radius, the length of gold wires, and the lattice constant are 16, 1532 and $70 \mathrm{~nm}$, respectively. The white arrow indicates the direction of power flow.

The minimum mean square fit does, in effect, average the field distribution on length scales small compared to the fitted effective wavelength. So the effective parameters are obtained for the averaged macroscopic field. The choice of the cross-section for the fit is arbitrary, but the results are practically independent on the location of the cross-section. 


\subsection{D Anisotropic Wire Medium}

The first structure for the 3D anisotropic wire medium in the optical region (Figure 3.3 (a)) is a $3 \mathrm{D}$ gold-wire square lattice with the wire radius, $r=16 \mathrm{~nm}$, and the lattice constant, $a=70 \mathrm{~nm}$, in vacuum. Figure 3.3 (b) shows that the group negative refraction occurs when a plane wave with the wavelength, $\lambda=700 \mathrm{~nm}$, and the transverse magnetic polarization, incidents on our simulated slab with an incident angle of $45^{0}$, while the phase velocity still undergoes a positive refraction. Pseudo focusing can also be seen from Figure 3.4, where the transverse magnetic polarized wave with the wavelength, $\lambda=700 \mathrm{~nm}$, coming out from a line source, is focused inside the simulated slab and then refocused on the other side of the slab.

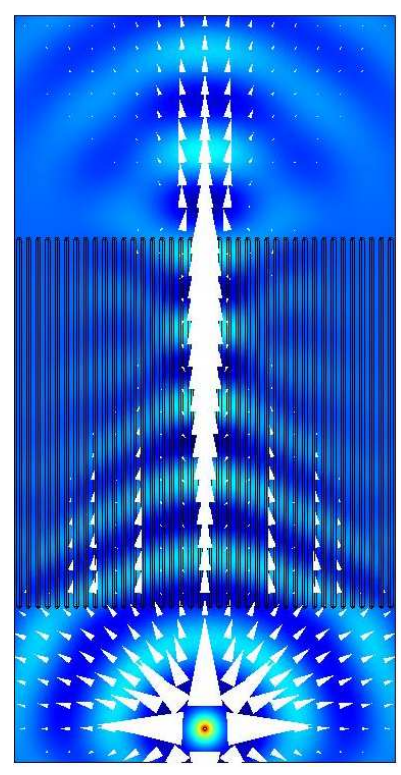

Figure 3.4 The magnetic field distribution of the pseudo focusing in a $3 \mathrm{D}$ gold-wire square-lattice medium with a line source placed $884 \mathrm{~nm}$ away from the interface, which launches a cylindrical transverse magnetic polarized wave at the wavelength $\lambda=700 \mathrm{~nm}$. The permittivity of gold is the same as in Figure 3.3. The background is vacuum. The radius, length of gold wires, and the lattice constant are 16, 2732 and $70 \mathrm{~nm}$, respectively. The white arrow indicates the direction of power flow.

When the geometric parameters, the wire radius $r=16 \mathrm{~nm}$, and the lattice constant $a=$

$70 \mathrm{~nm}$, are much smaller than the vacuum wavelength, $\lambda=700 \mathrm{~nm}$, of the incident EM wave, 
the 3D wire medium can be considered as a homogeneous effective medium [117-119]. The numerical dispersion relation of this 3D gold-wire square lattice medium is obtained and shown in Figure 3.5. The effective permittivities, $\varepsilon_{\perp}=-1.9082+i 0.2391$ and $\varepsilon_{\|}=1.4455+i 0.0044$, are obtained by fitting the numerical dispersion data into the hyperbolic dispersion relation [Eq. 3.1]. The fitted curve (dashed line) shows that the fitting is pretty good and the simulated metamaterial does have a hyperbolic dispersion relation.

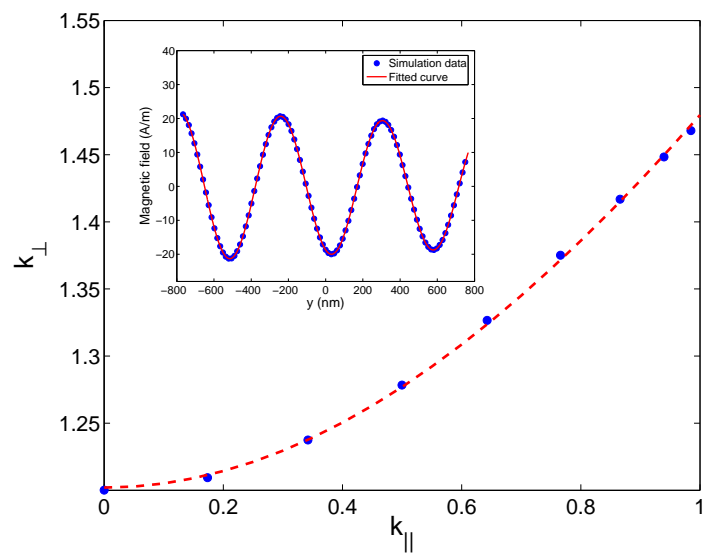

Figure 3.5 The numerical dispersion relation data from the simulation (solid circles) and the fitted hyperbolic curve (dashed line). All parameters are the same as in Figure 3.4, except the length of gold wires which is $1500 \mathrm{~nm}$. Note that all $k$ components here are normalized by $k_{0}$, where $k_{0}=\omega / c$. The inset shows, as a typical example, the field distribution for $\theta_{i}=30^{0}$ fitted by Eq. 3.2.

We have also used the Maxwell-Garnett equations $[118,119]$ to obtain the effective $\varepsilon_{\perp}$ and $\varepsilon_{\|}$at $\lambda=700 \mathrm{~nm}$ for different filling ratios for the square lattice of metallic wires. In Figure 3.6, we present the fitted results for $\varepsilon_{\|}$and $\varepsilon_{\perp}$ for different radii, while keeping the lattice constant unchanged. We use the following expressions for $\varepsilon_{\|}$and $\varepsilon_{\perp}$ from the Maxwell-Garnett theory:

$$
\begin{gathered}
\varepsilon_{\|}=\varepsilon_{d}\left[\frac{(1+f) \varepsilon_{m}+(1-f) \varepsilon_{d}}{(1-f) \varepsilon_{m}+(1+f) \varepsilon_{d}}\right] \\
\varepsilon_{\perp}=f \varepsilon_{m}+(1-f) \varepsilon_{d}
\end{gathered}
$$

where $f$ is the filling ratio of the metal, and $\varepsilon_{m}$ and $\varepsilon_{d}$ are the permittivities of metal and 


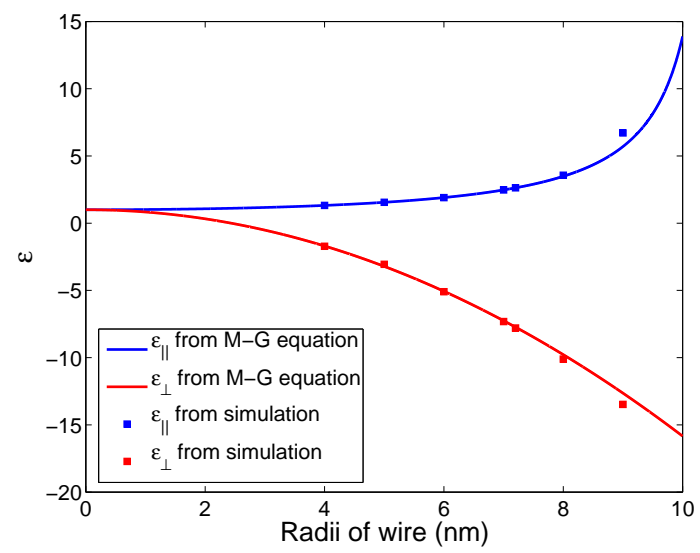

Figure 3.6 The effective permittivity $\varepsilon_{\perp}$ and $\varepsilon_{\|}$calculated from MaxwellGarnett equations (solid lines) and numerical simulations (squares) for different wire radii. The simulated medium is a $3 \mathrm{D}$ square lattice silver wire medium in vacuum with the lattice constant $a=20 \mathrm{~nm}$. The wavelength is $\lambda=700 \mathrm{~nm}$. The permittivity of silver at $\lambda=700 \mathrm{~nm}$ is $\varepsilon_{\text {silver }}=-20.4373+i 1.2863$, taken from experimental data [116].

dielectric, respectively. Notice that the effective values of $\varepsilon_{\perp}$ and $\varepsilon_{\|}$agree reasonably well with our fitting procedure. This is due to the effect that the vacuum wavelength, $\lambda=700 \mathrm{~nm}$, is much larger than the lattice constant and the radius of the metallic wires. In other cases, the effective parameters given by Eqs. 3.3 and 3.4 do not agree with our fitting procedure.

For comparison, we replace this $3 \mathrm{D}$ gold-wire square lattice medium slab with a homogeneous anisotropic slab with the fitted effective parameters $\varepsilon_{\|}=1.4455+i 0.0044$ and $\varepsilon_{\perp}=-1.9082+i$ 0.2391. (All other parameters are the same, such as the thickness and the width of the slab, the source and the distance between the source and the first interface, etc.) The simulation results for the magnetic field distribution and magnetic field intensity are shown in Figure 3.7. One can see that both of them have very good agreements between the homogeneous slab and the 3D wire medium. The excellent agreement proves again that our simulated 3D gold-wire square-lattice metamaterial indeed behaves as an effective medium, which has a hyperbolic dispersion relation and our fitting procedure works very well.

To be experimentally feasible, the second structure we examine is a hexagonal-lattice structure composed of silver wires in the alumina background. Figure 3.8 shows the magnetic field 


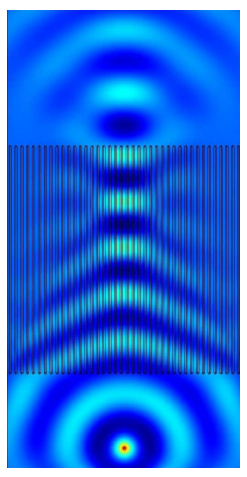

(a)

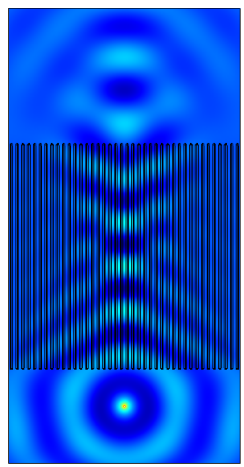

(e)

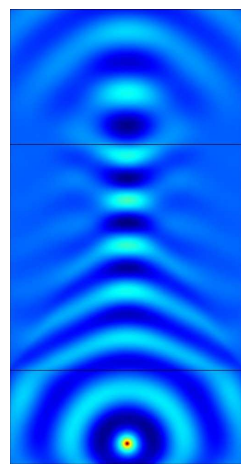

(b)

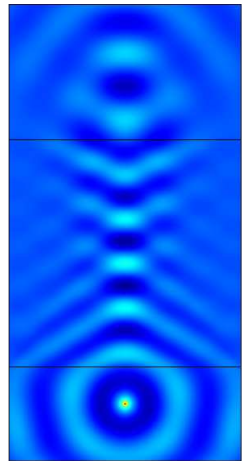

(f)

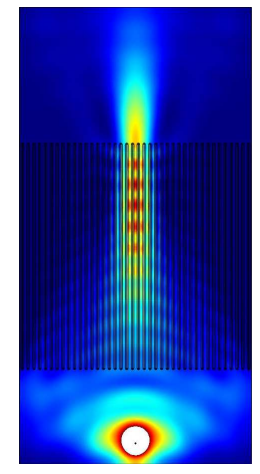

(c)

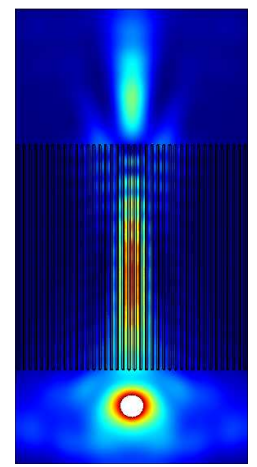

$(\mathrm{g})$

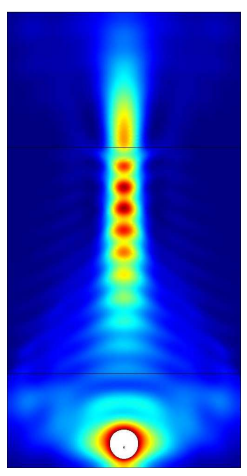

(d)

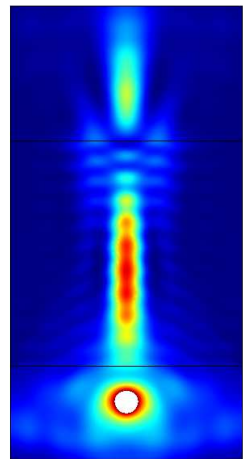

(h)

Figure 3.7 (a) The magnetic field distribution of the focusing simulation for the simulated 3D gold-wire square lattice anisotropic medium slab, with the source $884 \mathrm{~nm}$ away from the first interface. (b) Same as (a), but for a homogeneous anisotropic slab with the fitted effective parameters $\varepsilon_{\|}=1.4455+i 0.0044$ and $\varepsilon_{\perp}=-1.9082+i$ 0.2391. (c) and (d) are the same as (a) and (b), respectively, but for the magnetic field intensity distribution. (e) - (h) are the same as (a) - (d), respectively, except the source is $442 \mathrm{~nm}$ away from the first interface. All material parameters are the same as in Figure 3.4. 


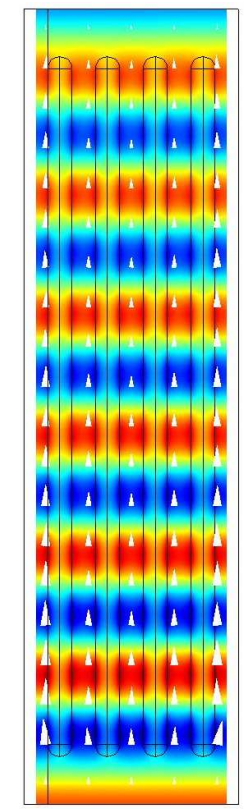

(a)

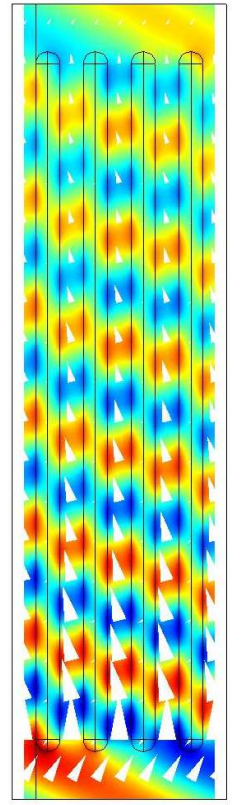

(b)

Figure 3.8 The magnetic field distribution in a 3D silver-wire hexagonal lattice medium slab with the alumina background. The incident plane wave has the transverse magnetic polarization and the wavelength in vacuum $\lambda=700 \mathrm{~nm}$. (a) Normal incidence. (b) At an incident angle of $30^{\circ}$. The white arrow indicates the direction of power flow. The hexagonal lattice constant $a$, the radius $r$, and the length $l$ of silver wires are $120 \mathrm{~nm}$, $30 \mathrm{~nm}$ and $1700 \mathrm{~nm}$, respectively. The permittivities of silver and alumina at the wavelength in vacuum $\lambda=700 \mathrm{~nm}$ are $\varepsilon_{\text {silver }}=-20.4373+i 1.2863$ and $\varepsilon_{\mathrm{Al}_{2} \mathrm{O}_{3}}=3.1$, respectively, taken from experimental data [116].

distributions along a cross-section perpendicular to the magnetic field for two different incident angles $\left(0^{0}\right.$ and $\left.30^{\circ}\right)$. For the incident angle $\theta=30^{0}$ case (Figure $\left.3.8(\mathrm{~b})\right)$, one can see that the group velocity (white arrow) undergoes a negative refraction inside the simulated medium. A substantial decay in the perpendicular direction for the magnetic field and the power flow exists for both of these two different incident angles (Figures 3.8(a) and 3.8(b)), since the lossy metallic wires have a very high filling ratio in this particular wire medium.

By the same fitting procedure, the numerical dispersion relation for the $3 \mathrm{D}$ silver-wire hexagonal lattice medium can also be obtained and is shown in Figure 3.9(a). The lowest 
four points are used to fit with a hyperbolic dispersion curve and the effective permittivity tensor is $\varepsilon_{\|}=5.3653+i 0.0708$ and $\varepsilon_{\perp}=-2.9188+i 0.4571$. One can see the large $k_{\|}$ points deviate from the fitted curve, even though the lowest four points are fitted very well. This occurs because we have a small wavelength/spatial period ratio of around 3.3 in alumina, which causes the breakdown of the homogeneous effective medium approximation in the large $k_{\|}$region.

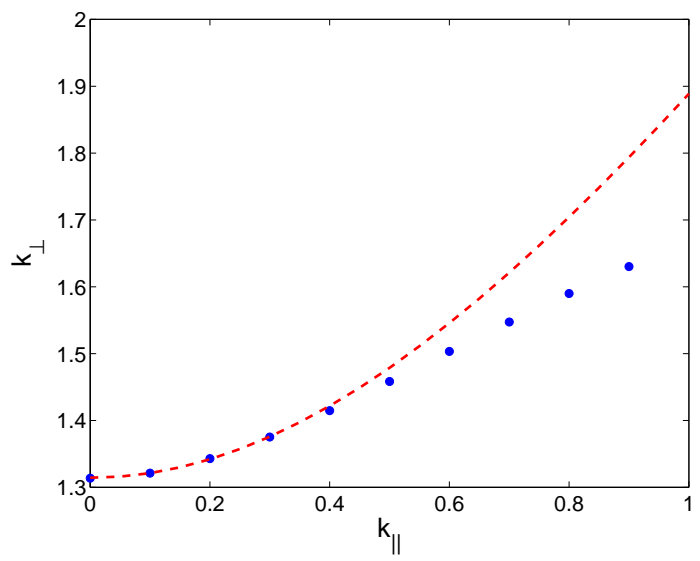

(a)

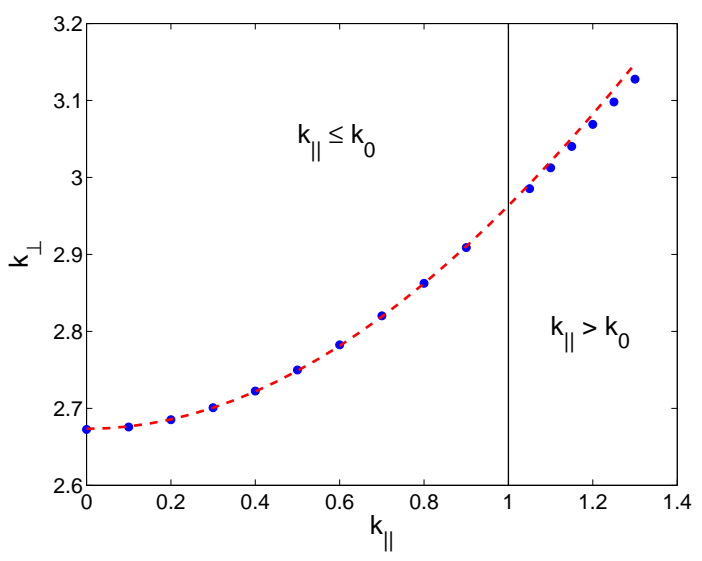

(b)

Figure 3.9 The numerical dispersion (solid circles) and the fitted dispersion curve (dashed line) of 3D silver-wire hexagonal-lattice media in the alumina background. (a) The lattice constant $a=120 \mathrm{~nm}$ and the radius of silver wires $r=30 \mathrm{~nm}$. (b) The lattice constant $a=30 \mathrm{~nm}$ and the radius of silver wires $r=12 \mathrm{~nm}$. $k_{\|} \leq k_{0}$ corresponds to the propagating modes in the background, while $k_{\|}>k_{0}$ corresponds to the evanescent modes. All other parameters are the same as in Figure 3.8. Note that all $k$ components are normalized by $k_{0}$, where $k_{0}=\sqrt{\varepsilon} \omega / c$ and $\varepsilon$ is the permittivity of alumina.

To extend the "good fitted" region to a larger $k_{\|}$range, where the numerical dispersion points can fit well into a hyperbolic dispersion curve, we reduce the hexagonal-lattice constant and the radius of silver wires to smaller values $a=30 \mathrm{~nm}$ and $r=12 \mathrm{~nm}$, respectively, while keeping all other parameters the same as before, so we can have a much higher wavelength/spatial period ratio of around 13. The fitted numerical dispersion relation is shown in Figure 3.9(b). The lowest ten points, which are propagating modes (i.e., 
$k_{\|} \leq k_{0}$, where $k_{0}=\sqrt{\varepsilon} \omega / c$ and $\varepsilon$ is the permittivity of alumina), are used to fit with a hyperbolic dispersion curve. The obtained effective permittivities are $\varepsilon_{\|}=22.1505+i 1.4693$ and $\varepsilon_{\perp}=-13.7714+i$ 0.6882. If we use Eqs. 3.3 and 3.4, the Maxwell-Garnett effective permittivities are given by $\varepsilon_{\|}=25.8371+i 2.0791$ and $\varepsilon_{\perp}=-10.5614+i 0.7466$, which do not agree well with our fitting parameters. Maxwell-Garnet equations are an approximation, in particular, known to fail completely for the usual wire metamaterials in the microwave regime. Here, we include the comparison of the effective parameters derived directly from the simulated field distribution with those in the Maxwell-Garnet approximation to show that for high frequency (low permittivity) and "thick" wires the Maxwell-Garnet approximation becomes good and can be used to guide design. (The reason for this is the domination of the electron mass over the magnetic effective mass for the electrons geometrically confined to the wires at near optical length scales and frequencies.) In Figure 3.9(b), one can also see that the numerical dispersion relation data from our fitting procedure are fitted very well into a hyperbolic dispersion curve, even for those large $k_{\|}$points, where $k_{\|}>k_{0}$. The latter are evanescent modes in air (and even in the alumina background of the wire medium), which are converted into propagating modes inside the slab and only attenuated by the losses of the effective medium. These modes preserve the information contained in the high spatial frequencies across the anisotropic slab and are essential for super-resolution applications.

\subsection{Conclusions}

We present two anisotropic metamaterials that demonstrate negative refraction and focusing. The first system is a superlattice of the metal-dielectric structure and the second system is (3D) metallic wires embedded in a dielectric matrix. We first obtain the numerical dispersion relation for the two cases by simulating the eigenmodes of the realistic system. The hyperbolic dispersion relation is obeyed in both cases, where the effective permittivities have opposite signs in the two propagation directions. Our simulations of the realistic structures, as well as the homogeneous simulations, show the negative refraction for all incident angles and demonstrate the focusing. The metallic nanowires can be valid for the evanescent modes in the 
dielectric background by having a large wavelength/spatial period ratio, which has important applications in super-resolution.

In conclusion, we numerically demonstrate that a homogeneous effective indefinite anisotropic medium can be realized by a 3D nanowire medium at the optical frequency region, which can have a negative refraction and pseudo focusing. We also present a nice fitting procedure by which we can obtain the numerical dispersion relation of our 3D wire medium and then retrieve its effective permittivity tensor. Meanwhile, we demonstrate that the hyperbolic dispersion relation of the $3 \mathrm{D}$ nanowire medium can be valid for the evanescent modes in the background by having a large wavelength/spatial period ratio (i.e., in the long wavelength limit), which may have important applications in super-resolution. 


\section{CHAPTER 4. SELF-CONSISTENT CALCULATIONS OF METAMATERIALS WITH GAIN}

\subsection{Introduction}

The field of metamaterials $[6,7]$ is driven by fascinating and far-reaching theoretical visions such as, e.g., perfect lenses [2], invisibility cloaking [36, 37], and enhanced optical nonlinearities $[39,40]$. This emerging field has seen spectacular experimental progress in recent years [5-10]. Yet, losses are orders of magnitude too large for the envisioned applications. For some applications, such as perfect absorbers [120-122], the loss is not a problem. Although some indefinite materials, such as the metallic wire medium, can reduce the losses by avoiding the resonances, most metamaterials are constituted by an array of resonators. To reduce the large resonance losses, there are two basic approaches. One approach to reduce the metamaterial losses to some extent is by geometric tailoring of the metamaterial designs [28, 64, 66, 67]. An efficient method to geometrically reduce the losses in metamaterials is by increasing the inductance, $L$, to the capacitance, $C$, ratio [64], and avoid corners and sharp edges in metamaterials [67]. Another method to reduce losses is to move the real part of the negative index of refraction, $n$, away from the maximum of $\operatorname{Im}(n)$ (close to the resonance) $[28,66]$ by strongly coupled metamaterials. However, achieving significant enough loss reduction by further design optimization appears to be out of reach. Thus, the second approach, incorporation of active media (gain) into metamaterial designs, might come as a cure. The dream would be to simply inject an electrical current into the active medium, leading to gain and hence to compensation of the losses. One important issue is not to assume the metamaterial layer and the gain medium layer are independent from one another $[68,72,74-79,123]$. So, experiments on such intricate active nanostructures do need guidance by theory via self-consistent calculations [80-83] (using 
the semi-classical theory of lasing) for realistic gain materials that can be incorporated into or close to dispersive media to reduce the losses at $\mathrm{THz}$ or optical frequencies. The need for self-consistent calculations stems from the fact that increasing the gain in the metamaterial, the metamaterial properties change, in turn changes the coupling to the gain medium until a steady-state is reached. A specific geometry to overcome the severe loss problem of optical metamaterials and to enable bulk metamaterials with negative magnetic and electric response and controllable dispersion at optical frequencies is to interleave active material layers with the passive metamaterial lattice.

For reference, the best fabricated negative-index material operating at around $1.4 \mu \mathrm{m}$ wavelength [29] has shown a figure of merit, $\mathrm{FOM}=-\operatorname{Re}(n) / \operatorname{Im}(n) \approx 3$, where $n$ is the effective refractive index. This experimental result is equivalent to an absolute absorption coefficient of $\alpha=3 \times 10^{4} \mathrm{~cm}^{-1}$, which is even larger than the absorption of typical direct-gap semiconductors such as, e.g., GaAs (where $\alpha=10^{4} \mathrm{~cm}^{-1}$ ). So it looks difficult to compensate the losses with this simple type of analysis, which assumes that the bulk gain coefficient is needed. However, the effective gain coefficient, derived from self-consistent microscopic calculations, is a more appropriate measure of the combined system of metamaterial and gain. Due to pronounced local-field enhancement effects in the spatial vicinity of the dispersive metamaterial, the effective gain coefficient can be substantially larger than its bulk counterpart. With regard to experiments to reduce losses in metamaterials, one needs to use semiconductor gain (quantum dots or wells) and not use dye molecules [84], which photo-bleach rapidly. Semiconductor gain enables long-term use and can be conceptually pumped by electrical injection. This is crucial, as applications based on optically-pumped structures do not appear to be realistic in the long run. However, to check if losses in metamaterials can be reduced experimentally, one can try exploratory experiments under conditions of optical pumping. While early models [68, 74, 123-125] using simplified gain-mechanisms such as explicitly forcing negative imaginary parts of the local gain material's response function produce unrealistic strictly linear gain, our self-consistent approach presented below allows for determining the range of parameters for which one can realistically expect linear amplification and linear loss compensation in the 
metamaterial [80-82]. The approximation in these earlier models can effectively be obtained in our approach by assuming a constant population inversion in Eq. 2.26, i.e., a population inversion given by an "average field". To fully understand the coupled metamaterial-gain system, we have to deal with time-dependent wave equations in metamaterial systems by coupling Maxwell's equations with the rate equations of electron populations describing a multi-level gain system in semi-classical theory [89].

In this chapter, we aim to to apply a detailed computational model to the problem of metamaterials with gain, which is described by a generic four-level atomic system (see section 2.2.3 in Chapter 2). The total electron density of gain materials is chosen to be $N_{0}(t=$ $0)=5.0 \times 10^{23} / \mathrm{m}^{3}$ and the lifetimes of the third, second and first levels, $\tau_{32}, \tau_{21}$ and $\tau_{10}$ are chosen $5 \times 10^{-14}, 5 \times 10^{-12}$ and $5 \times 10^{-14} \mathrm{~s}$, respectively. The coupling strength $\sigma_{a}$ of the atomic transition between the second level $\left(N_{2}\right)$ and the first level $\left(N_{1}\right)$ is chosen $10^{-4} \mathrm{C}^{2} / \mathrm{kg}$, however, its linewidth $\Gamma_{a}$ and center frequency $\omega_{a}$ may vary for different simulations. For optical pumping, the center frequency $\omega_{b}$, the linewidth $\Gamma_{b}$ and the coupling strength $\sigma_{b}$ of the atomic transition between the ground state level $\left(N_{0}\right)$ and the third level $\left(N_{3}\right)$ are $4 \pi \times 10^{14} \mathrm{~Hz}$, $2 \pi \times 10 \times 10^{12} \mathrm{~Hz}$ and $5 \times 10^{-6} \mathrm{C}^{2} / \mathrm{kg}$, respectively. In order to solve the behavior of the gain materials in the electromagnetic fields numerically, the finite-difference time-domain (FDTD) method is utilized $[81-83,87,88,90,91]$. The initial condition is that all electrons are in the ground state and all electric, magnetic and polarization fields are zero. Then the electrons are pumped from $N_{0}$ to $N_{3}$ optically or with a homogeneous pumping rate $\Gamma_{\text {pump }}$. The system begins to evolve according to the Maxwell's equations, rate equations and driven oscillator equations. In section 4.2 , we verify that our code agrees well with simple soluble models (gain material only). In addition, our code is applied to $1 \mathrm{D}$ gain slab and $1 \mathrm{D}$ superlattice of gain and negative index layers. The results for the lasing and nonlinear behaviors are presented. In section 4.3, loss compensation in 2D metamaterials is considered. Results are presented for a square lattice of Lorentz dielectric cylinders with layers of gain material and a 2D split ring resonator (SRR) with gain material inclusion. The losses can be compensated by gain and lasing (spasing) is achieved in our numerical simulations. In sections 4.4 and 4.5 , the gain is 


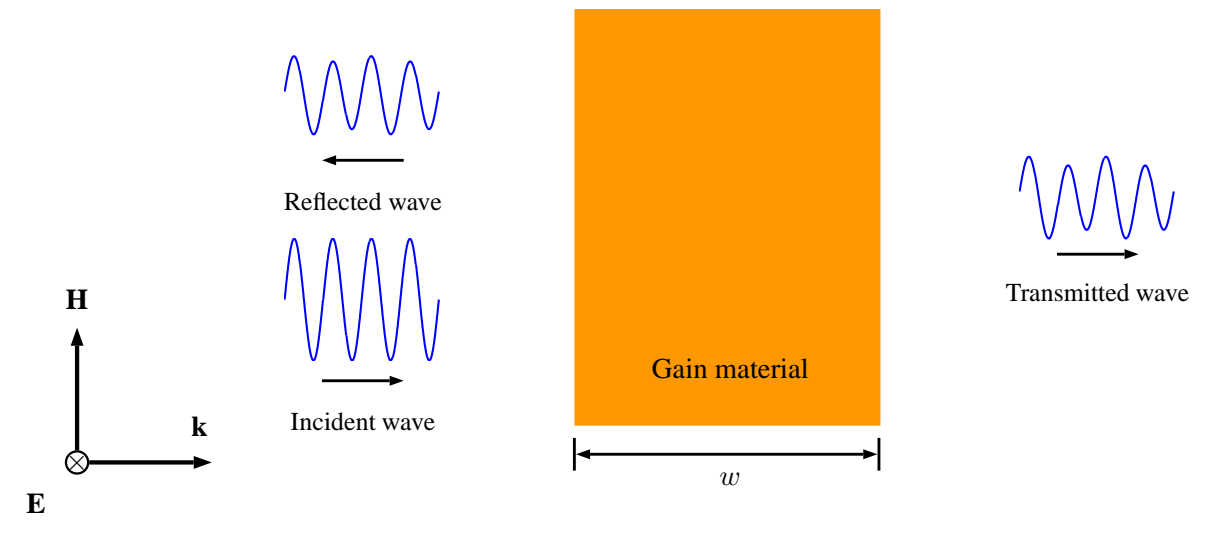

Figure 4.1 Schematic of gain material slab (shown in orange). The slab width $w$ takes different values in the cases we have examined.

embedded in two different realistic metamaterials - a fishnet structure and an array of 3D SRRs. The simulation results show the large resonance losses can be effectively compensated by the inclusion of gain in these realistic metamaterials due to the strong local field enhancement.

\subsection{Lasing and Nonlinear Behaviors in Gain Materials and Metamaterials Incorporated with Gain}

\subsubsection{Gain material only}

To understand the lasing behavior of gain material, we first study a gain material slab surrounded by vacuum (shown in Figure 4.1). The center frequency $\omega_{a}$ and the linewidth $\Gamma_{a}$ of the transition between $N_{1}$ and $N_{2}$ are chosen 100 and $5 \mathrm{THz}$, respectively. And the discrete time and space steps are chosen to be $\Delta t=1.67 \times 10^{-17} \mathrm{~s}$ and $\Delta x=1.0 \times 10^{-8} \mathrm{~m}$, respectively. We generate a continuous wave $(\mathrm{CW})$ at the frequency $\omega_{b}(200 \mathrm{THz})$ and let it propagate through the gain slab, and then we calculate the reflected and transmitted waves and implement the Fourier transforms to see if there is lasing and how much power is emitted around $100 \mathrm{THz}-$ the emission frequency $\omega_{a}$ between $N_{1}$ and $N_{2}$. First we start with a very low input power $P_{\text {in }}$ for the incident $\mathrm{CW}$ wave, but no lasing happens, then we increase the input power till it reaches the lasing threshold, for which the system starts to have lasing and we can see a small peak at the emission frequency $100 \mathrm{THz}$ in the Fourier transforms of reflected and transmitted 


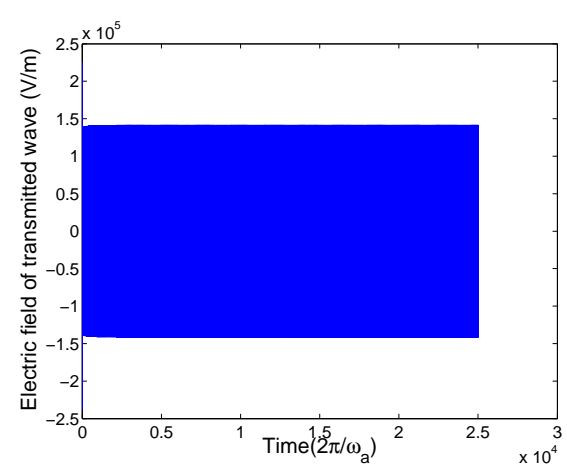

(a)

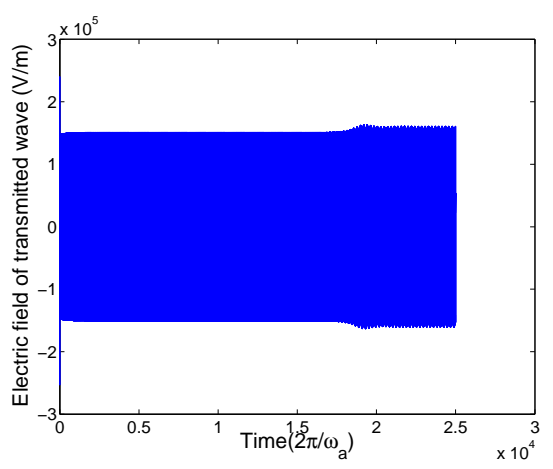

(c)

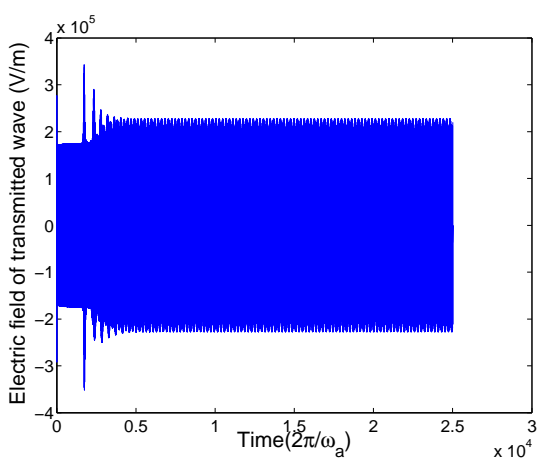

(e)

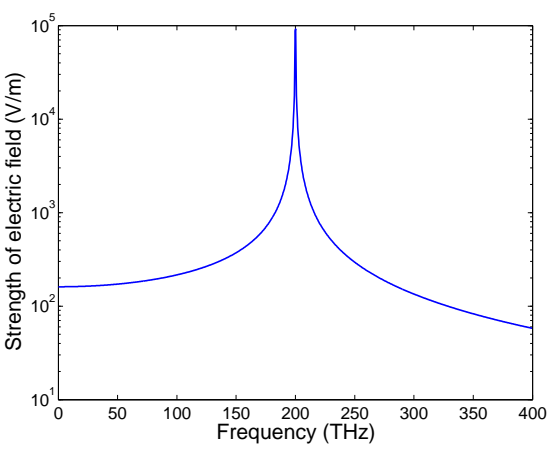

(b)

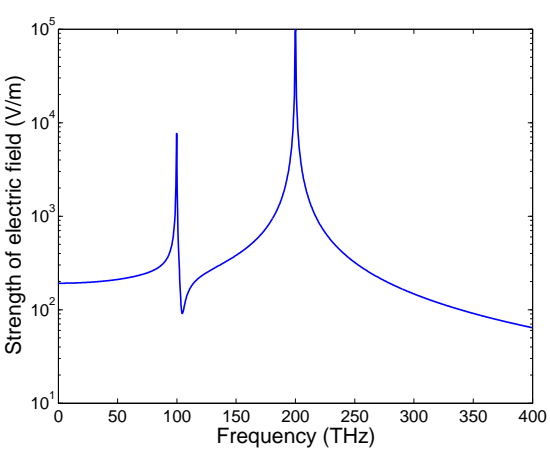

(d)

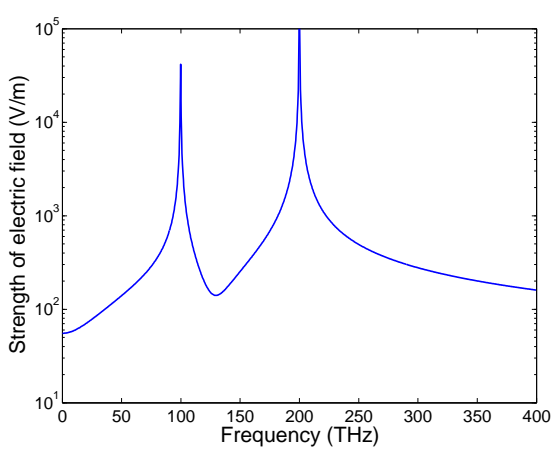

(f)

Figure 4.2 The transmitted waves and their corresponding Fourier transforms for different input powers. (a), (c) and (e) are the transmitted waves for input power $P_{\text {in }}=79.6,90.7$ and $120.6 \mathrm{~W} / \mathrm{mm}^{2}$, respectively. (b), (d) and (f) are same as (a), (c) and (e), respectively, but for the Fourier transforms of the transmitted waves. The gain slab width $w=100 \mathrm{~nm}$ and the bandwidth $\Gamma_{a}$ of the atomic transition between $N_{1}$ and $N_{2}$ is $5 \mathrm{THz}$. 
waves, i.e., low power emitted around the emission frequency $\omega_{a}$. If we keep increasing the input power, the peak will get higher and the emitted power will get larger. Figure 4.2 shows the transmitted waves and their corresponding Fourier transforms for three different input powers at the gain slab $w=100 \mathrm{~nm}$. We can see there is no lasing (Figure 4.2(a)) when the input power is low $\left(P_{\text {in }}=79.6 \mathrm{~W} / \mathrm{mm}^{2}\right)$ and there is only one peak for the pumping frequency in its Fourier transform(Figure $4.2(\mathrm{~b})$ ). When the input power $P_{\text {in }}=90.7 \mathrm{~W} / \mathrm{mm}^{2}$, the system starts lasing (Figure 4.2(c)) and a small peak appears at the frequency $100 \mathrm{THz}$ (Figure 4.2(d)). If we increase the input power to a higher value $P_{\text {in }}=120.6 \mathrm{~W} / \mathrm{mm}^{2}$, the lasing gets stronger (Figure 4.2(e)) and the peak for the emission frequency gets higher (Figure 4.2(f)), i.e., more power emitted around the emission frequency $\omega_{a}$. We have calculated the emitted power at the emission frequency $\omega_{a}$ versus the input power at the pumping frequency $\omega_{b}$ for the same gain slab system. As shown in Figure 4.3, we can see that there is a sharp rise in the emission around $P_{\text {in }} \approx 90.7 \mathrm{~W} / \mathrm{mm}^{2}$, which corresponds to the lasing threshold for this system. Below the threshold, there is no lasing.

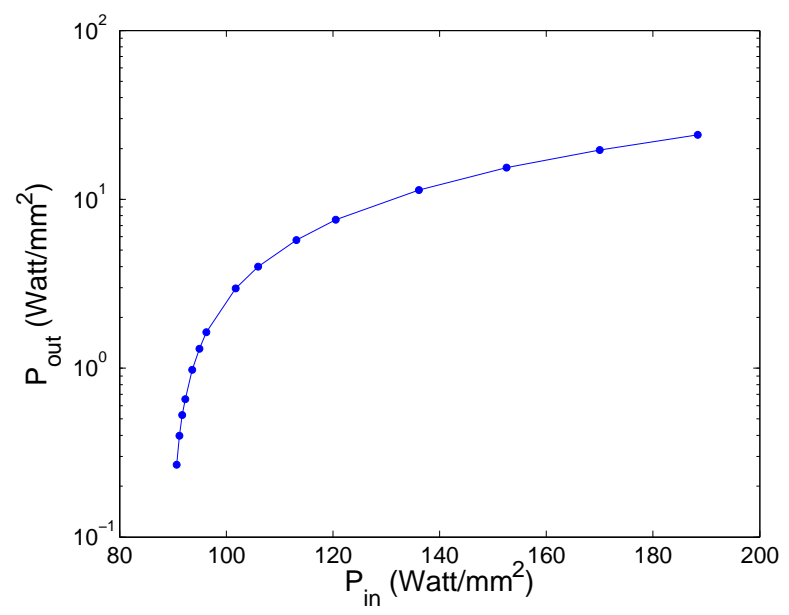

Figure 4.3 The powers emitted at the emission frequency $\omega=\omega_{a}(100 \mathrm{THz})$ for different input powers at the pumping frequency $\omega=\omega_{b}$ $(200 \mathrm{THz})$. All parameters of this system are same as Figure 4.2.

We also notice that the lasing time (the time when the system starts lasing) varies according to the input power. Figure 4.4 shows the detailed results for the lasing time versus the input 
power with the slab widths $w=100,250$ and $500 \mathrm{~nm}$. We can see the lasing time decreases as the input power increases because the system pumps the electrons at a higher rate from the ground state level to higher levels and then reaches the population inversion between $N_{1}$ and $N_{2}$ in a shorter time. For a fixed input power, one can see the lasing time decreases as the gain slab width gets larger. This occurs because more input energy is absorbed and then converted to lasing by the wider gain slab.

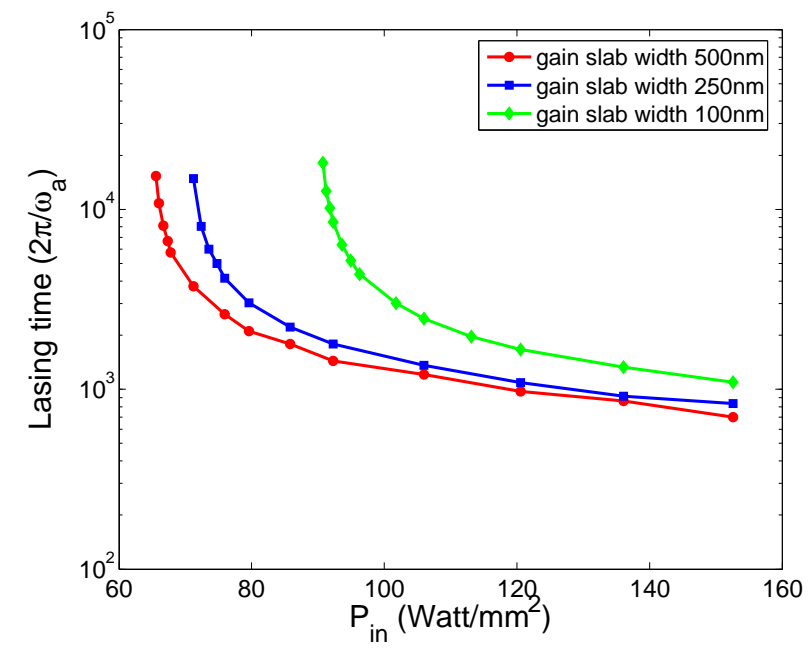

Figure 4.4 The lasing times for different input powers at the pumping frequency $\omega_{b}(200 \mathrm{THz})$. The gain slab width $w=100,250$ and $500 \mathrm{~nm}$, respectively. All other parameters are same as Figure 4.2 .

As the input wave propagates inside the gain slab, it will decay due to the absorption from the gain material at the pumping frequency $\omega_{b}$ (see Figure 4.5). Thus the pumping rate, which is determined by the local input optical intensity, is inhomogeneous inside the gain slab. But for a thin gain material layer, the electric field of the input wave can be approximately treated as homogeneous, thus we can simplify the pumping process between $N_{0}$ and $N_{3}$ by using a homogeneous pumping rate $\Gamma_{\text {pump }}$. For a very thin gain slab $w=100 \mathrm{~nm}$, simulations are done with a homogeneous pumping rate and the results for the power emitted around the emission frequency $\omega_{a}$ versus the pumping rate are plotted in Figure 4.6. Comparing with Figure 4.3, where the electrons are optically pumped, we can see they are very similar. For a 


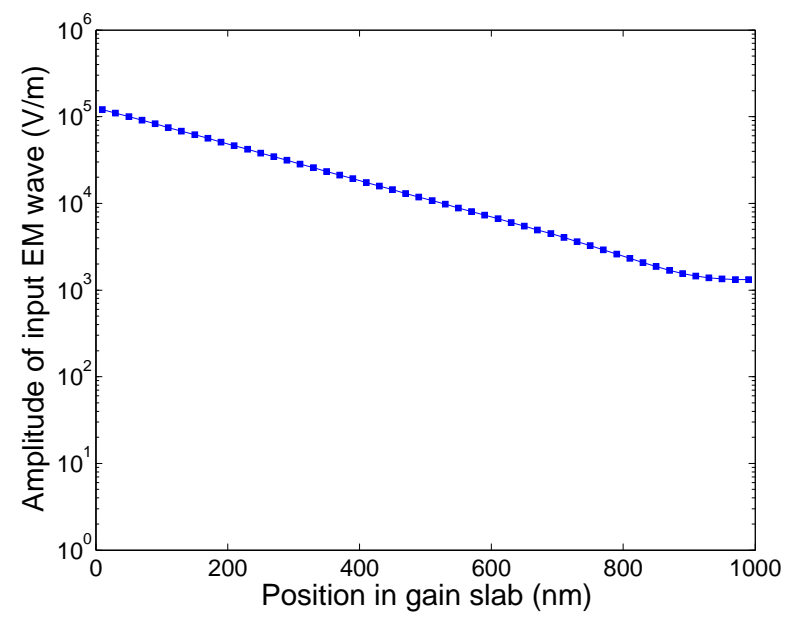

Figure 4.5 The amplitude of the input EM wave inside the gain slab as a function of the position. The gain slab width $w=1000 \mathrm{~nm}$ and the input power $P_{\text {in }}=92.3 \mathrm{~W} / \mathrm{mm}^{2}$. All other parameters are same as Figure 4.2 .

fixed output power such as $P_{\text {out }}=7.56 \mathrm{~W} / \mathrm{mm}^{2}$, we can find the corresponding input power $P_{\text {in }}=120.6 \mathrm{~W} / \mathrm{mm}^{2}$ in Figure 4.3 and the corresponding pumping rate $\Gamma_{\text {pump }}=9.3 \times 10^{9} \mathrm{~s}^{-1}$ in Figure 4.6. Then we do simulations for optical pumping case with the input power $P_{\text {in }}=$ $120.6 \mathrm{~W} / \mathrm{mm}^{2}$ and for the homogeneous pumping rate case with the pumping rate $\Gamma_{\text {pump }}=$ $9.3 \times 10^{9} \mathrm{~s}^{-1}$. The graphs of the occupation numbers as a function of time are plotted in Figure 4.7 for both cases. One can see that they are almost the same. This verifies that the homogeneous pumping rate simplification is valid for a thin gain slab. In our following simulations, we will use this simplification because the gain slab widths in our structures are very thin $(w<=50 \mathrm{~nm})$.

\subsubsection{Negative index material (NIM) embedded in layers of gain}

As the first simple model system, we will discuss a one-dimensional metamaterial system which consists of layers of negative index material (NIM) and gain material, as shown in Figure 4.8, to see if we can compensate the losses of the metamaterials associated with the NIMs by the amplification provided by the gain material layers and how the system starts lasing. The gain material and the discrete time and space steps are the same as section 4.2.1. 


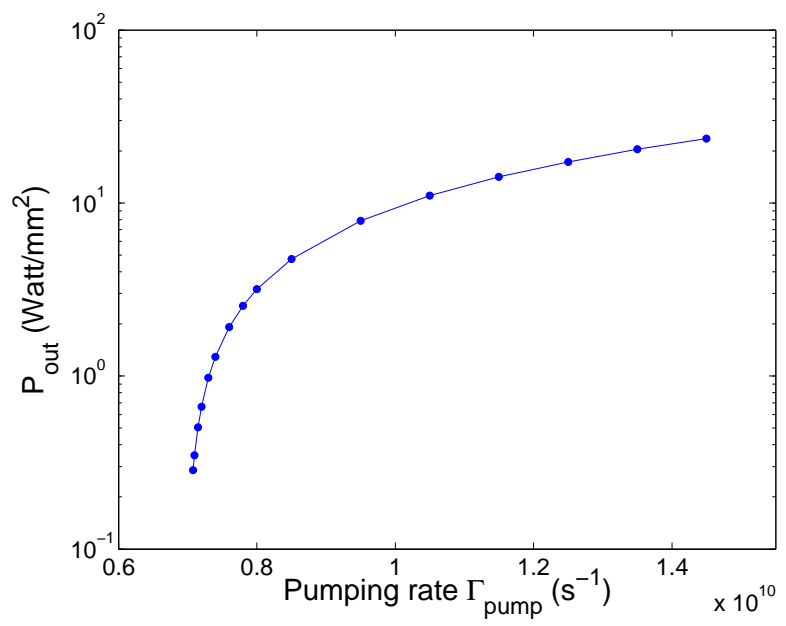

Figure 4.6 The powers emitted at the emission frequency $\omega=\omega_{a}(100 \mathrm{THz})$ for different pumping rates. The gain slab width $w=100 \mathrm{~nm}$ and the bandwidth of the atomic transition between $N_{1}$ and $N_{2}$ is $5 \mathrm{THz}$.

We first let a narrow band Gaussian pulse of a given amplitude go through the metamaterial without gain, and we calculate the transmitted signal emerging from the metamaterial system, which also has a Gaussian profile but its amplitude is much smaller than that of the incident pulse due to the losses of NIM layers. Then we introduce the gain into the system and start increasing the pumping rate. The amplitude of the transmitted signal gets larger and we can find a critical pumping rate, for which the transmitted pulse is of the same amplitude as the incident one. Since the gain material itself is nonlinear, we increase the amplitude of the incident Gaussian pulse for a fixed pumping rate to see how high we can go in the strength of the incident electric field and still have the full compensation of the losses, i.e., the transmitted signal equals the incident signal, independent on the signal strength. In this region we have compensated loss and still have linear response of the metamaterial. The shape of the transmitted signal is only affected by the dispersion but not dependent on the signal strength. For a three-layer system (NIM - gain material - NIM), we have calculated the transmission versus the strength of the electric field of the incident signal for several pumping rates close to the critical pumping rate $\Gamma_{\text {pump }}=4.70 \times 10^{9} \mathrm{~s}^{-1}$, as shown in Figure 4.9. We find it has a linear response within a very broad range up to incident electric field of $10^{3} \mathrm{~V} / \mathrm{m}$. If we use 


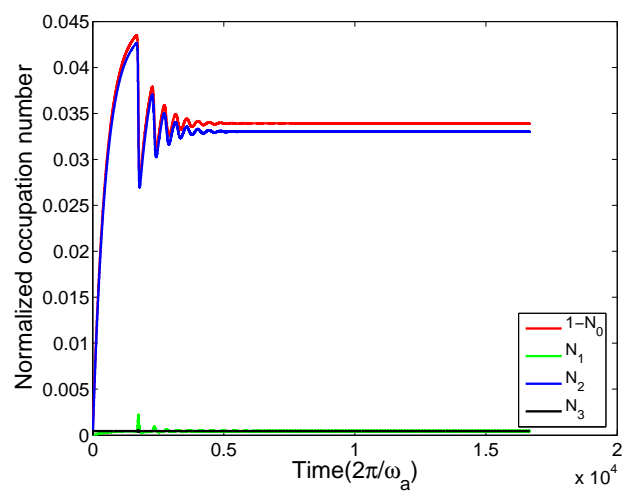

(a)

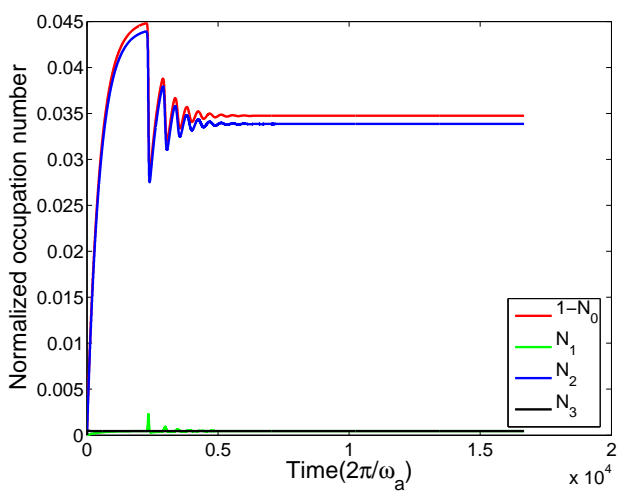

(b)

Figure 4.7 The normalized occupation numbers as a function of time. The gain slab width $w=100 \mathrm{~nm}$ and the gain bandwidth of the atomic transition between $N_{1}$ and $N_{2}$ is $5 \mathrm{THz}$. (a) The electrons are optically pumped by an input EM wave with input power $P_{\text {in }}=120.6 \mathrm{~W} / \mathrm{mm}^{2}$ and (b) the electrons are pumped with a homogeneous pumping rate $\Gamma_{\text {pump }}=9.3 \times 10^{9} \mathrm{~s}^{-1}$. Occupation numbers $N_{0}, N_{1}, N_{2}$ and $N_{3}$ are normalized by the total electron density $N_{i}\left[N_{i}=N_{0}(t=0)=5.0 \times 10^{23} / \mathrm{m}^{3}\right]$.

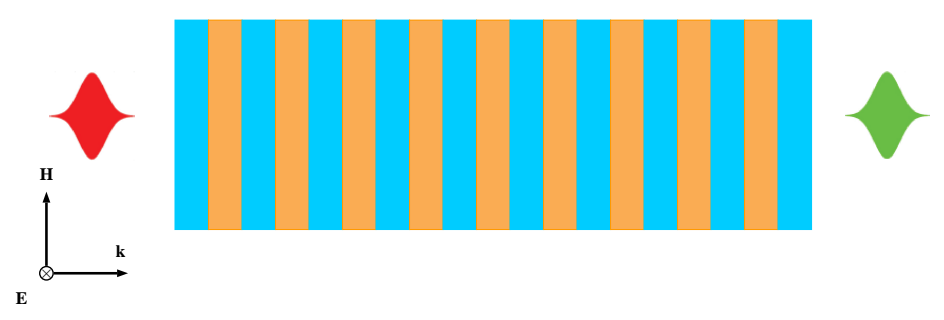

Figure 4.8 The negative index material (blue) embedded in layers of gain material (orange). The number of layers, the permittivity and permeability of NIM are taken different values for different cases we have examined. The width for both NIM and gain material is $w=50 \mathrm{~nm}$. The gain bandwidth is $5 \mathrm{THz}$. 


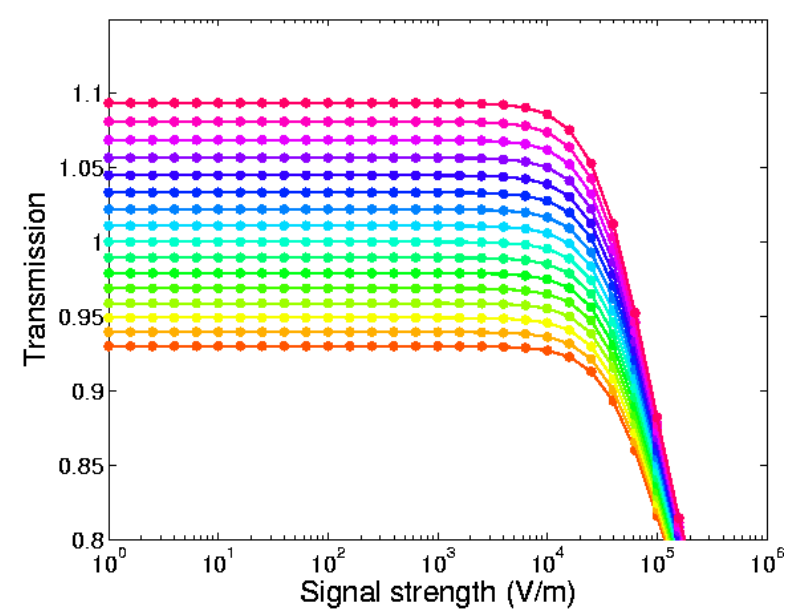

Figure 4.9 The transmission vs. signal amplitude for the loss-compensated metamaterial of a three-layer system (NIM - gain material - NIM) with gain bandwidth of $5 \mathrm{THz}$, for different pumping rates $\Gamma_{\text {pump }}$. $\Gamma_{\text {pump }}$ is increased from $4.0 \times 10^{9} \mathrm{~s}^{-1}$ (lowest) to $5.5 \times 10^{9} \mathrm{~s}^{-1}$ (highest) in steps of $1.0 \times 10^{8} \mathrm{~s}^{-1}$. The material parameters for NIM are $\varepsilon=\mu=-1+2 i$. The metamaterial response is linear in a very wide range. When the loss-compensated transmission reaches exactly unity, the pumping rate $\Gamma_{\text {pump }}=4.70 \times 10^{9} \mathrm{~s}^{-1}$, which is called the critical pumping rate. For incident fields stronger than $10^{4} \mathrm{~V} / \mathrm{m}$ the metamaterial behaves nonlinearly.

19 layers of Figure 4.8, the critical pumping rate is $1.98 \times 10^{9} \mathrm{~s}^{-1}$, which is even smaller than half of the three-layer case, and the linear regime becomes narrower and drops faster than the 3-layer case for higher strength of incident electric field (shown in Figure 4.10). To include the nonlinearity of gain material for strong incident signal, it is necessary to do a self-consistent calculation using FDTD method.

As an example, we have also studied the three-layer system with different losses, to see how much $\Gamma_{\text {pump }}$ we need to compensate the losses. Figure 4.11 shows there exists a linear relation between the critical pumping rate and the imaginary part of the refractive index $n$ of NIMs.

We have also numerically calculated the susceptibilities of the gain material to see if it really has a Lorentz lineshape. We first let a Gaussian pulse of a given amplitude $(10 \mathrm{~V} / \mathrm{m})$ propagate through the metamaterial system and calculate the time-domain electric polarization 


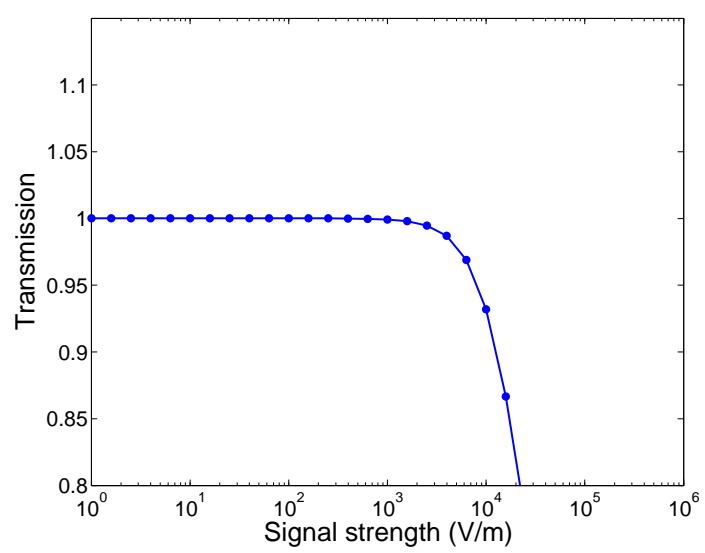

Figure 4.10 The transmission vs. signal amplitude for the loss-compensated metamaterial of Figure 4.8 with gain bandwidth of $5 \mathrm{THz}$ at the critical pumping rates $\Gamma_{\text {pump }}=1.98 \times 10^{9} \mathrm{~s}^{-1}$. The material parameters for NIM are same as Figure 4.9. For incident fields stronger than $10^{3} \mathrm{~V} / \mathrm{m}$ this metamaterial becomes nonlinear.

$\mathbf{P}(\mathbf{r}, t)$ and the local electric field $\mathbf{E}(\mathbf{r}, t)$. Then we implement the Fourier transforms to obtain the frequency-domain polarization and electric fields and calculate the frequency-dependent susceptibility by using the equation $\chi^{\prime}(\omega)+i \chi^{\prime \prime}(\omega)=P(\omega) / \varepsilon_{0} E(\omega)$. Simulations are done for both 3 and 19 layers and results are compared with the analytic results calculated using the equations [89] $\chi^{\prime}=-\chi_{0}^{\prime \prime} \Delta x /\left(1+\Delta x^{2}\right)$ and $\chi^{\prime \prime}=\chi_{0}^{\prime \prime} /\left(1+\Delta x^{2}\right)$ with $\Delta x=2\left(\omega-\omega_{a}\right) / \Gamma_{a}$ and $\chi_{0}^{\prime \prime}=-\sigma_{a} \Delta N /\left(\varepsilon_{0} \omega_{a} \Gamma_{a}\right)$, where $\Delta N=N_{2}-N_{1}$. As shown in Figure 4.12, we find that the numerical susceptibilities are the same as the analytic ones and they do have a Lorentz lineshape.

To understand the lasing behavior of the metamaterial system, we increase the pumping rate to provide more gain from the gain material. We found the amplification of the incident signal gets larger and at last the system starts lasing till the pumping rate reaches a certain high value. Figure 4.13(a) shows the lasing behavior at the pumping rate $\Gamma_{\text {pump }}=1.5 \times 10^{10} \mathrm{~s}^{-1}$ and there appears a peak at the emission frequency $100 \mathrm{THz}$ in the corresponding Fourier transform (Figure 4.13(b)).

If we use real metal layers instead of negative index materials, we can not compensate the losses of the metals. The reason is that the permittivity $\varepsilon$ for metals is large and negative and 


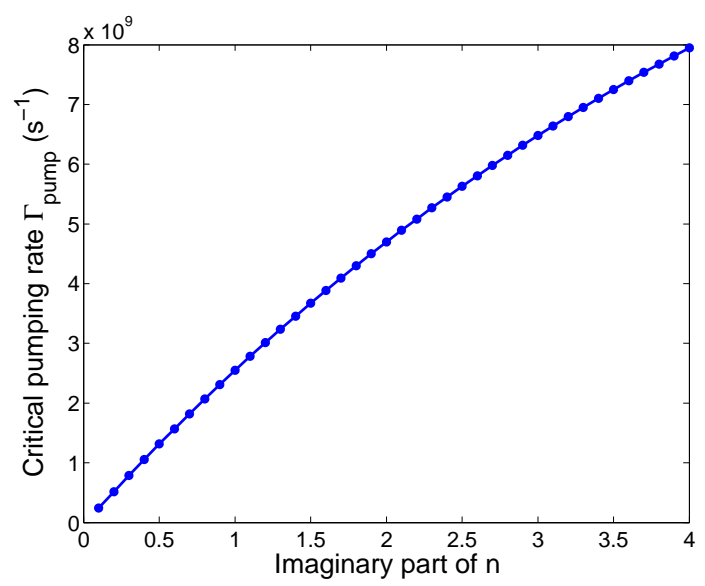

Figure 4.11 The critical pumping rates for different imaginary parts of the refractive index $n$ of NIMs. The structure is a three-layer system (NIM - gain material - NIM) and $\varepsilon=\mu$ for NIMs.

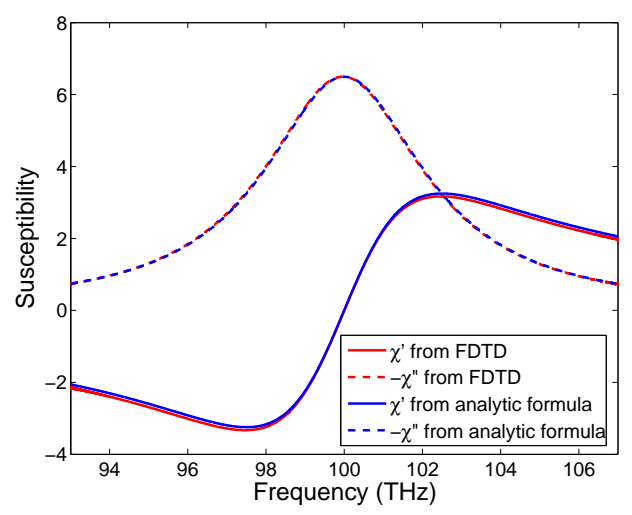

(a)

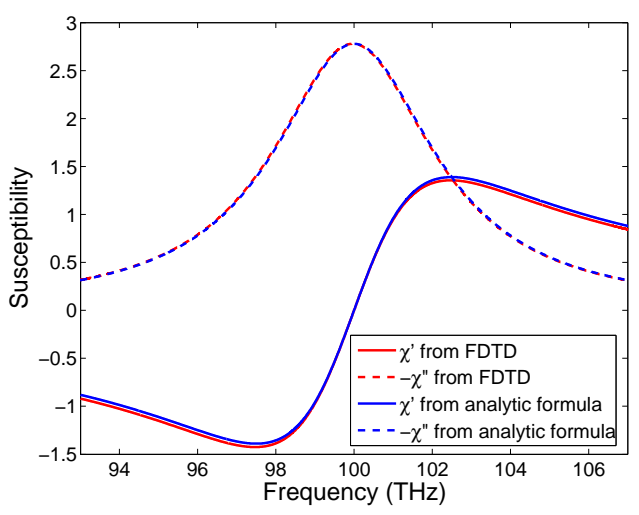

(b)

Figure 4.12 The numerical and analytical results for the susceptibilities of gain materials as a function of frequency. (a) a three-layer system (NIM - gain material -NIM) at the critical pumping rate $\Gamma_{\text {pump }}=4.7 \times 10^{9} \mathrm{~s}^{-1}$. (b) a 19-layer system of Figure 4.8 at the critical pumping rate $\Gamma_{\text {pump }}=1.98 \times 10^{9} \mathrm{~s}^{-1}$. 


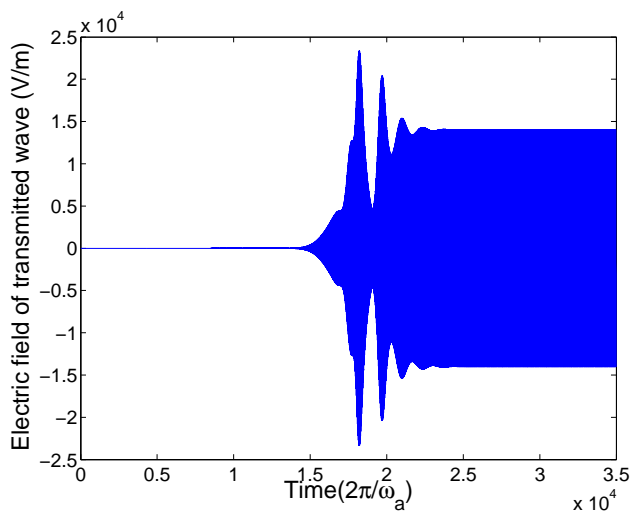

(a)

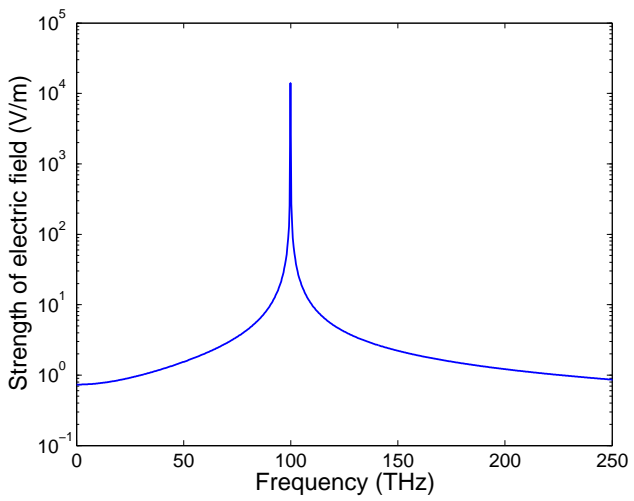

(b)

Figure 4.13 (a) The time-dependent electric field of the transmitted wave for a three-layer system (NIM - gain material - NIM) and (b) the corresponding Fourier transform in frequency domain for the lasing in (a). The pumping rate $\Gamma_{\text {pump }}=1.5 \times 10^{10} \mathrm{~s}^{-1}$.

we'll have large reflections due to the impedance mismatch.

\subsubsection{Conclusions}

We have performed numerical simulations on one dimensional gain material system by using FDTD method. The system starts lasing when the input reaches over the lasing threshold. For greater input power/a wider gain slab, the lasing is faster. Comparisons were done for a gain material slab between the optical pump method and its homogeneous pumping rate simplification and results show that this simplification can be valid for a thin gain slab.

A one-dimensional metamaterial system consisted of layers of negative index material (NIM) and gain material is also studied using FDTD method. We numerically show the losses of NIM can be compensated by the amplification of the gain material layers. The nonlinear behaviors are studied by increasing the amplitude of the incident Gaussian pulse for a fixed pumping rate. There is a relatively wide range of input signal amplitudes where the NIM-gain system behaves linearly. When the amplitudes get higher, the system becomes nonlinear, due to the nonlinearity of the gain material itself. It is necessary to have self-consistent calculations to determine the signal range where we can expect a linear response. Further, if we have strong signals, so that we are in nonlinear regime, or we want to study lasing, the 
self-consistent calculation is needed. We also show the critical pumping rate, for which the loss-compensated transmission reaches exactly unity, has a linear relation with the imaginary part of the refractive index $n$ of NIMs. In addition, we verified our FDTD code by comparing the numerically calculated susceptibility of the gain material and its analytical counterpart, and studied the lasing behavior of the NIM-gain system by increasing the pumping rate.

\subsection{Loss Compensation in 2D Metamaterials}

In section 4.2 .2 , we simply force the permittivity and the permeability of the metamaterial to be negative to have an unrealistic negative index material. In this section, we consider two-dimensional (2D) metamaterial systems to emulate the resonant elements in a realistic metamaterial.

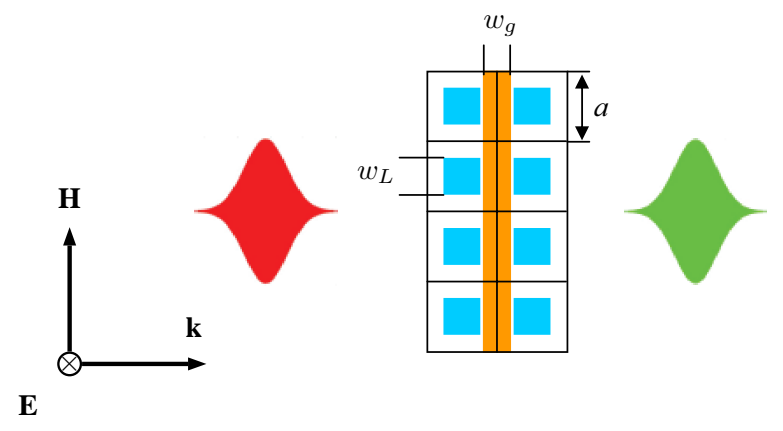

Figure 4.14 One layer of gain material (orange) embedded in a square lattice of dielectric square cylinders (blue) that have a Lorentz behavior. The dielectric constant of the cylinders is given by $\varepsilon=1+\omega_{p}^{2} /\left(\omega_{p}^{2}-2 i \omega \gamma-\omega^{2}\right)$, where the resonance frequency $f_{p}=\omega_{p} / 2 \pi=100 \mathrm{THz}$ and $\gamma=2 \pi f$, and $f$ takes different values in the cases we have examined. The dimensions are $a=80 \mathrm{~nm}, w_{L}=40 \mathrm{~nm}$, and $w_{g}=30 \mathrm{~nm}$.

\subsubsection{One layer of gain material embedded in a square lattice of Lorentz dielectric cylinders}

The first system is consisted of one layer of gain material and two layers of dielectric wires that have a Lorentz-type resonant electric response to emulate the electric resonator, such as 


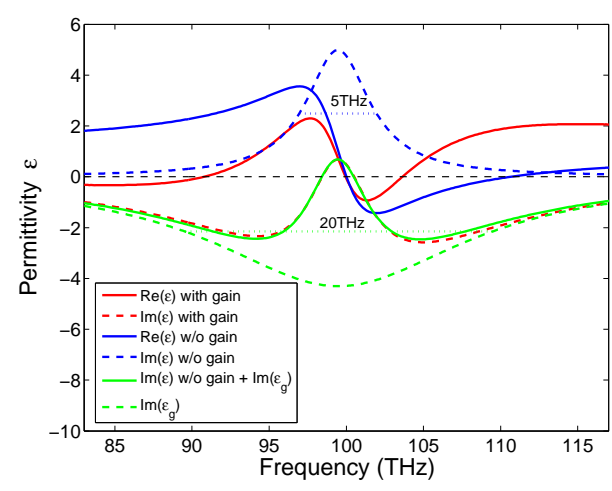

(a)

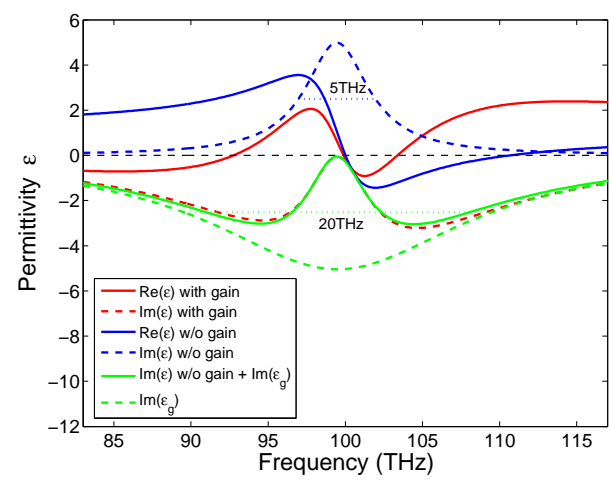

(c)

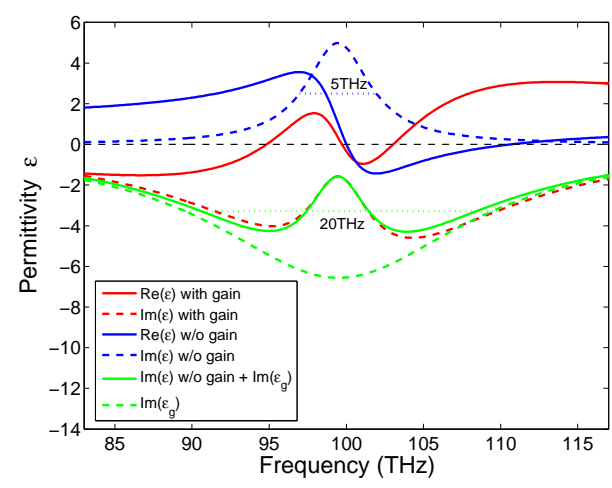

(e)

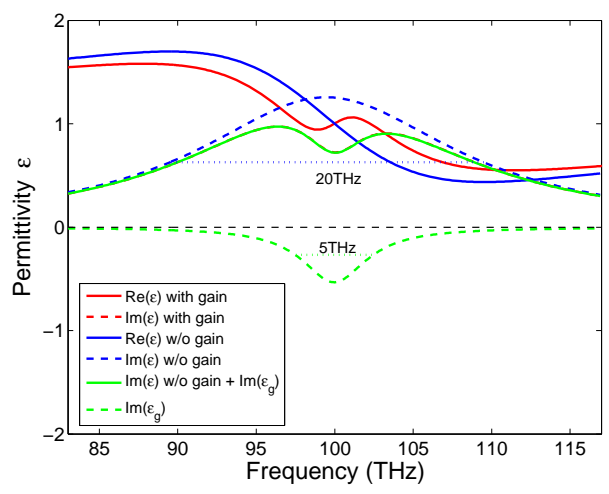

(b)

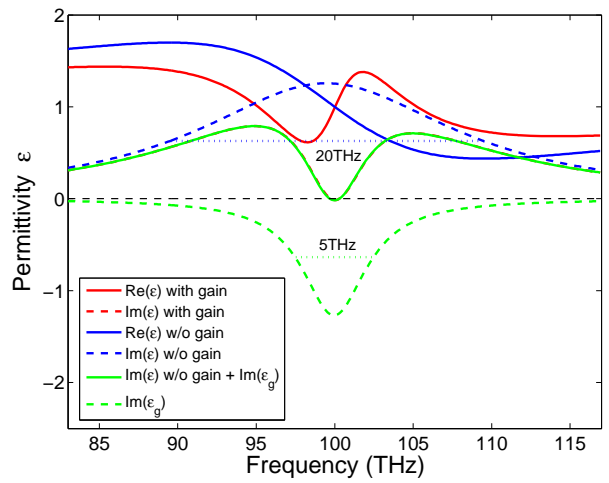

(d)

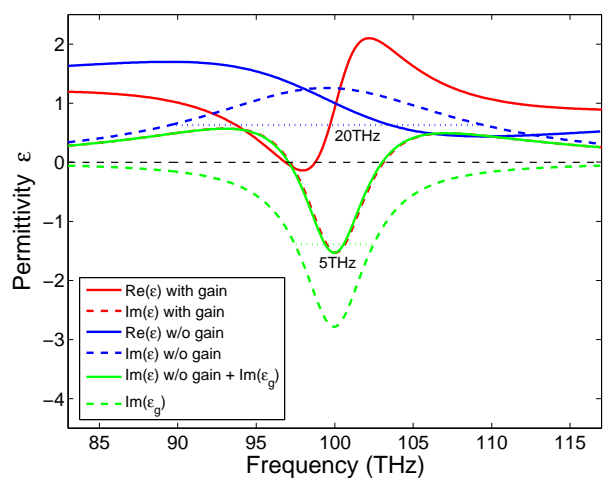

(f)

Figure 4.15 (a) The retrieved results for the real and the imaginary parts of the effective permittivity $\varepsilon$ with gain and without gain. Below compensation, $t=0.89$; gain and Lorentz bandwidths are $20 \mathrm{THz}$ and $5 \mathrm{THz}$, respectively. (c) and (e) are same as (a), but for the loss compensated case $(t=1)$ and the overcompensated case $(t=1.34)$, respectively. (b), (d) and (f) are same as (a), (c) and (e), respectively, except with gain bandwidth $5 \mathrm{THz}$ and Lorentz bandwidth $20 \mathrm{THz}$. 
cut-wires, as shown in Figure 4.14. The center radiation frequency of the gain material is $100 \mathrm{THz}$. And the discrete time and space steps are $8.33 \times 10^{-18} \mathrm{~s}$ and $5.0 \times 10^{-9} \mathrm{~m}$, respectively. We first study the three-layer system with the full width at half maximum (FWHM) $5 \mathrm{THz}$ for Lorentz dielectric (i.e., $f=2.5 \mathrm{THz}$ ) and $20 \mathrm{THz}$ for gain. The transmission, $T$, the reflection, $R$, and the absorption, $A=1-T-R$, as a function of frequency for the system are obtained in the propagation direction. With the introduction of gain, the absorption at the resonance frequency of $100 \mathrm{THz}$ decreases and reaches 0 at a certain pumping rate. So the gain compensates the losses. If we continue increasing the gain, i.e., the pumping rate, the system gets overcompensated and the absorption becomes negative. To see how the losses of the emulated resonators get compensated by the gain, we exploit the usual retrieval procedure based on inverting the scattering amplitudes [126] to obtain the effective permittivities $\varepsilon$ without gain and with gain. Figure 4.15(a), (c) and (e) show the retrieved results for the real and the imaginary parts of the effective permittivities $\varepsilon$ of the system for the below compensation, loss-compensated, and overcompensated cases, respectively, together with the effective permittivity without gain. The retrieved results for $\varepsilon$ without gain have exactly the Lorentz shape but the amplitude of the real and the imaginary parts of $\varepsilon$ is a factor of 4 less than the Lorentz formula for the square cylinders. This is due to the filling ratio of the square cylinders in the unit cell. Due to the loss compensation from the gain material, one can see the imaginary part of the effective permittivity gets lower as the gain increases. Below compensation, its value at the resonance frequency of $100 \mathrm{THz}$ is positive, while it's zero and negative for the loss-compensated and overcompensated cases, respectively. Notice that we can have $\operatorname{Re}(\varepsilon) \approx 2$ with $\operatorname{Im}(\varepsilon) \approx 0$ at $98 \mathrm{THz}$ and $\operatorname{Re}(\varepsilon) \approx-1$ with $\operatorname{Im}(\varepsilon) \approx 0$ at $101 \mathrm{THz}$ for the below compensation case (Figure 4.15(a)). For the loss-compensated case (Figure 4.15(c)), we have $\operatorname{Re}(\varepsilon) \approx 1$ with $\operatorname{Im}(\varepsilon) \approx 0$ at the resonance frequency $100 \mathrm{THz}$, which is just same as vacuum and makes no sense for us. For the overcompensated case (Figure 4.15(e)), the imaginary part of the effective permittivity $\varepsilon$ is negative within all the frequency range.

Second, we study the three-layer system with the FWHM of gain smaller than Lorentz dielectric, where the bandwidths for gain and Lorentz dielectric are $5 \mathrm{THz}$ and $20 \mathrm{THz}$ (i.e., 
$f=10 \mathrm{THz}$ ), respectively. The introduction of gain develops a peak at the resonance frequency of $100 \mathrm{THz}$ for the transmission while the absorption has a dip. The retrieved results for the real and the imaginary parts of the effective permittivities $\varepsilon$ without gain and with gain are plotted in Figure 4.15(b), (d) and (f) for the three different cases discussed above. Similar to the first case we examined where the loss bandwidth is smaller than the gain, the imaginary part of the effective permittivity $\varepsilon$ gets smaller due to the gain. The difference is we get interesting results for the overcompensated case instead of the below compensation case, where we can have $\operatorname{Re}(\varepsilon) \approx 0$ with $\operatorname{Im}(\varepsilon) \approx 0$ at $97 \mathrm{THz}$ and $\operatorname{Re}(\varepsilon) \approx 2.1$ with $\operatorname{Im}(\varepsilon) \approx 0$ at $103 \mathrm{THz}$.

So for both the two systems, one can obtain a lossless metamaterial with positive or negative $\operatorname{Re}(\varepsilon)$, either below compensation or over compensation. In Figure 4.15 we also have plotted the sum of $\operatorname{Im}(\varepsilon)$ without gain and the imaginary part of $\varepsilon_{g}$, the dielectric function of the gain material. One can see the imaginary part of $\varepsilon$ of our total system with gain is equal to the sum of $\operatorname{Im}(\varepsilon)$ and $\operatorname{Im}\left(\varepsilon_{g}\right)$. This is unexpected because there is no coupling between the Lorentz dielectric and the gain. This is indeed true for the 2D Lorentz dielectric cylinders, because they have a continuous shape like a solenoid and the gain material slabs have zero depolarization field. Different from finite length wires [hence a three-dimensional (3D) problem] where the dipole interactions between Lorentz wires and gain material are dominated by the near field $O\left(1 / r^{3}\right)$, the interaction for infinite length wires is only via the propagating field $O(\omega \ln |k r|)$, and much weaker. That's why the Lorentz wires and the gain material are approximately independent in our $2 \mathrm{D}$ simulations. So there is a need for a true $3 \mathrm{D}$ simulation to solve this problem and obtain different behaviors. However, the $3 \mathrm{D}$ simulation is computationally excessively demanding.

Like the layered system in section 4.2 .2 , if we keep increasing the pumping rate, i.e., the gain, at last both of the two systems will have lasing. For example, when the pumping rate reaches $\Gamma_{\text {pump }}=3.2 \times 10^{10} \mathrm{~s}^{-1}$, the three-layer system with gain bandwidth $5 \mathrm{THz}$ and Lorentz bandwidth $20 \mathrm{THz}$ starts lasing — the system itself has a coherent self-sustained steady output. 


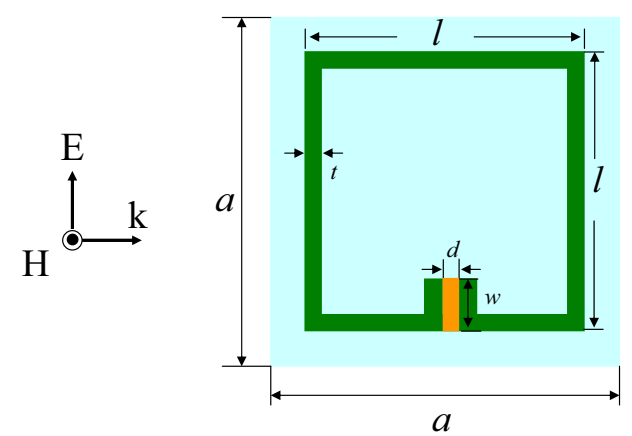

Figure 4.16 Geometry for a unit cell of the square SRR system with gain embedded in the gap (orange). The dimensions are $a=100 \mathrm{~nm}, l=80 \mathrm{~nm}, t=5 \mathrm{~nm}, d=5 \mathrm{~nm}$ and $w=15 \mathrm{~nm}$.

\subsubsection{D split ring resonators (SRRs) with gain material inclusions}

To avoid the decoupling problem in section 4.3.1 and still limit our simulations in a $2 \mathrm{D}$ model, we consider a 2D split ring resonator (SRR) as a more realistic and also more relevant model, where the relevant polarization is across the finite SRR gap and therefore the coupling to the gain material is dipole-like. In Figure 4.16, we show the geometry for the unit cell of the SRR system with the gain material embedded in the gap. The dimensions of the SRR are chosen such that the magnetic resonance frequency of the SRR overlaps with the emission frequency $(100 \mathrm{THz})$ of the gain material. The discrete time and space steps are chosen $8.33 \times 10^{-19} \mathrm{~s}$ and $1.0 \times 10^{-9} \mathrm{~m}$, respectively. Due to the strong electric field inside the gap, there will be strong coupling between the SRR and the gain material. We also want to see if the losses of the magnetic response can be compensated by the electric gain.

Simulations are done for one layer of the square SRR with gain bandwidth of $20 \mathrm{THz}$. Figure 4.17(a) shows the retrieved results for the real and the imaginary parts of the effective permeability $\mu$, with gain (pumping rate $\Gamma_{\text {pump }}=1.0 \times 10^{9} \mathrm{~s}^{-1}$ ) and without gain. One can see that with the introduction of gain, the gain undamps the magnetic resonance of the SRR and the weak and broad resonant effective permeability $\mu$ of the lossy SRR becomes strong and narrow. The FWHM with gain is $2.61 \mathrm{THz}$, while the FWHM without gain is $5.85 \mathrm{THz}$, which is more than twice larger than the former. Notice that in the off-resonance range in Figure 4.17(a), 


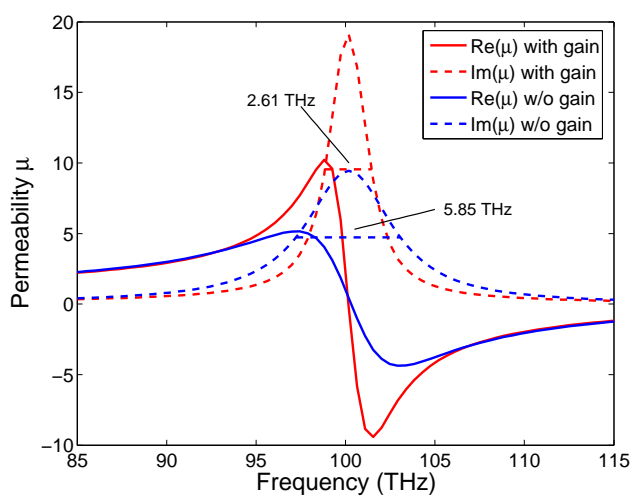

(a)

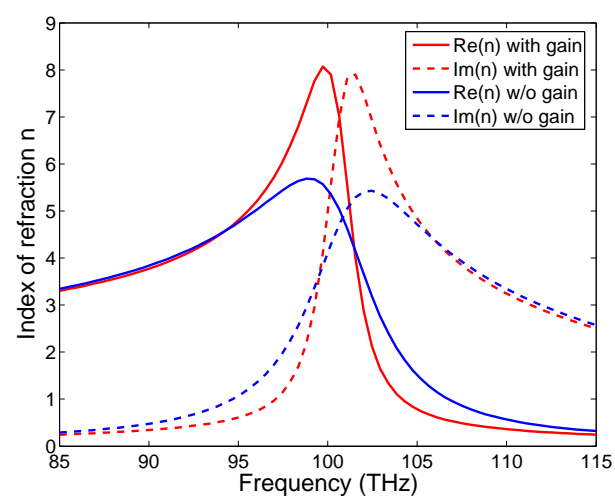

(b)

Figure 4.17 The retrieved results for the real and the imaginary parts of (a) the effective permeability $\mu$ and (b) the corresponding effective index of refraction $n$, with and without gain for a pumping rate $\Gamma_{\text {pump }}=1.0 \times 10^{9} \mathrm{~s}^{-1}$ and the SRR system of Figure 4.16. The gain bandwidth is $20 \mathrm{THz}$. Notice that the width of the magnetic resonance with gain is $2.61 \mathrm{THz}$.

we can obtain the effective permeability $\mu$ with a smaller imaginary part with the introduction of the gain, which means the magnetic loss is compensated by the electric gain. Figure 4.17(b) shows the retrieved results for the real and the imaginary parts of the corresponding effective index of refraction $n$, with and without gain. Note that for a lossless $\mathrm{SRR}, n$ is purely real away from the resonance except in a small band above the resonance where it's purely imaginary due to the negative $\mu$. At the frequency of $96 \mathrm{THz}$, slightly below the resonance (Figure 4.17(b)), the imaginary parts of the index of refraction $n$ without and with gain are 1.36 and 0.754 . Then we can find the effective extinction coefficient without gain is $\alpha=(\omega / c) \operatorname{Im}(n) \approx 2.74 \times 10^{4} \mathrm{~cm}^{-1}$ and the one with gain is $\alpha \approx 1.52 \times 10^{4} \mathrm{~cm}^{-1}$. And hence the effective amplification coefficient of the gain in the combined system is $\alpha \approx-1.22 \times 10^{4} \mathrm{~cm}^{-1}$, which is much larger than the amplification $\alpha \approx-9.2 \times 10^{2} \mathrm{~cm}^{-1}$ for the bulk gain material [89] at the given pumping rate $\Gamma_{\text {pump }}=1.0 \times 10^{9} \mathrm{~s}^{-1}$. This is due to the strong local electric field enhancement in the gap of the resonant SRR. While we have the incident electric field $10 \mathrm{~V} / \mathrm{m}$, the induced electric field in the gap is around $450 \mathrm{~V} / \mathrm{m}$. Taking the observed field enhancement factor around 45 in the gap of SRR, the energy produced by the gain in the gap is around 12 times larger 
than from the homogeneous bulk gain material in the size of the unit cell, which agrees well with the factor around 15 between the simulated SRR effective medium and the homogeneous gain medium. If we continue increasing the pumping rate, the magnetic resonance becomes narrower $\left(0.96 \mathrm{THz}\right.$ for pumping rate $\left.\Gamma_{\text {pump }}=1.8 \times 10^{9} \mathrm{~s}^{-1}\right)$. When the pumping rate reaches $\Gamma_{\text {pump }}=1.9 \times 10^{9} \mathrm{~s}^{-1}$, the metamaterial system gets overcompensated and the imaginary part of the effective permeability $\mu$ at the resonance frequency gets flipped down and becomes negative. If we increase the pumping rate even more (around $\Gamma_{\text {pump }}=5.0 \times 10^{9} \mathrm{~s}^{-1}$ ), the SRR system starts lasing $[73,127]$.

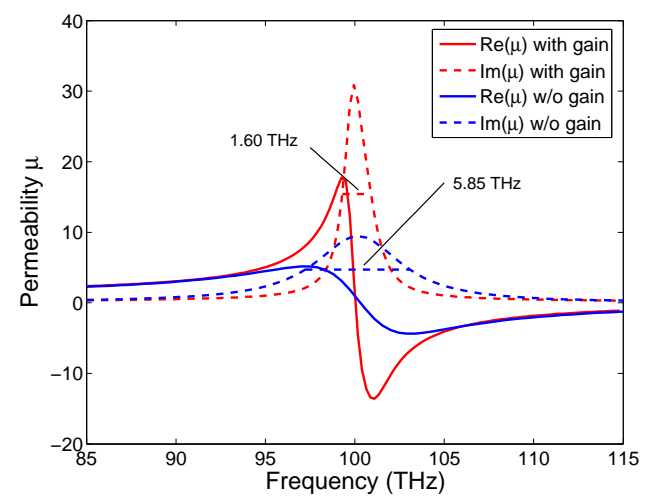

Figure 4.18 The retrieved results for the real and the imaginary parts of the effective permeability $\mu$ with and without gain for a pumping rate $\Gamma_{\text {pump }}=8.0 \times 10^{8} \mathrm{~s}^{-1}$ and the $\mathrm{SRR}$ system where the SRR is surrounded by gain. The gain bandwidth is $20 \mathrm{THz}$. Notice that the width of the magnetic resonance with gain is 1.60 THz, narrower than Figure 4.17(a).

Instead of having the gain in the gap of SRR, we also have done simulations on the SRR system where the gain is surrounding around the SRR. The retrieved results for the real and the imaginary parts of the effective permeability $\mu$ without gain and with gain for a given pumping rate $\Gamma_{\text {pump }}=8.0 \times 10^{8} \mathrm{~s}^{-1}$ are plotted in Figure 4.18. Due to the larger gain filling in the unit cell, the coupling between the gain material and the SRR gets stronger and the losses of SRR are easier to be compensated. Compared with the case with the gain in the gap only, the effective permeability $\mu$ of the simulated SRR system in Figure 4.18 can have stronger and narrower resonance $(\mathrm{FWHM}=1.6 \mathrm{THz})$ even with lower pumping rate. Similar to the previous 
case, the magnetic resonance becomes stronger and narrower $\left(0.7 \mathrm{THz}\right.$ at $\left.\Gamma_{\text {pump }}=1.0 \times 10^{9} \mathrm{~s}^{-1}\right)$ as we increase the pumping rate, and the system gets overcompensated so that the magnetic resonance peak gets flipped down when the pumping rate reaches $\Gamma_{\text {pump }}=1.1 \times 10^{9} \mathrm{~s}^{-1}$. It's also easier for this system to have lasing [73, 127], which is observed when the pumping rate is $\approx \Gamma_{\text {pump }}=2.5 \times 10^{9} \mathrm{~s}^{-1}$.

\subsubsection{Conclusions}

Numerical simulations have been done for $2 \mathrm{D}$ metamaterial systems - a square lattice of Lorentz dielectric cylinders with layers of gain material and a 2D SRR with gain material inclusion. The results show that the losses of $2 \mathrm{D}$ metamaterials can be compensated by the gain material.

For the 2D Lorentz dielectric wire lattice, there is no coupling between the Lorentz dielectric wires and the gain material, due to the infinite length of the wires, which leads to zero depolarization field in the gain material. This is unexpected and implies the need for a true $3 \mathrm{D}$ simulation for this problem.

The 2D SRR has the relevant polarization across its gap and therefore can strongly couple to the gain embedded in its gap. Two SRR systems with different gain inclusions were studied in the linear region of the gain. We have demonstrated that the magnetic losses of the SRRs can be easily compensated by the electric gain by investigating the retrieved permeability and index of refraction. The magnetic resonance gets stronger and narrower with the pumping rate increasing. The pumping rate needed to compensate the losses is much smaller than the bulk gain material due to the strong local field enhancement in the gap of the SRRs. The losses of

the SRR surrounded by gain can be easier to be compensated than the SRR with gain in the gap only due to more coupling with the gain. Provided that the pumping rate is high enough, the metamaterial nanostructures can have lasing. 


\subsection{Overcoming Losses with Gain in Fishnet Metamaterials}

In section 4.3 , the loss compensation of $2 \mathrm{D}$ metamaterials is studied, however, the realistic metamaterial structure is in three dimensions. So there is a need to study the loss compensation of 3D metamaterials to guide the experimentalists to seek new 3D metamaterial designs. As the first example, we present numerical results for loss compensation of the fishnet structure at optical frequencies. This is important, because the experimentalists in the metamaterial community are working on compensating the losses by introducing gain in the fishnet metamaterials [84].

\subsubsection{Geometric dimensions of the fishnet structure}

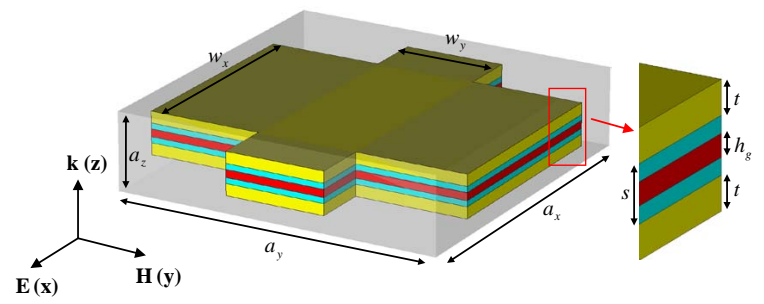

Figure 4.19 Schematic of the unit cell of the fishnet structure with the parameters marked on it. The geometric parameters are $a_{x}=a_{y}=860 \mathrm{~nm}, a_{z}=200 \mathrm{~nm}, w_{x}=565 \mathrm{~nm}, w_{y}=265 \mathrm{~nm}$, $s=50 \mathrm{~nm}, t=30 \mathrm{~nm}$ and $h_{g}=20 \mathrm{~nm}$. The thicknesses of the metal (silver) and gain layer are $t$ and $h_{g}$, respectively, and the dielectric constant of the spacer, $\mathrm{MgF}_{2}$, is 1.9. These parameters were used on simulations [Ref. [128]] and experiments [Ref. [28]].

In Figure 4.19, we show the unit cell of the fishnet structure. The size of the unit cell along the propagation direction is $a_{z} . a_{z}$ is larger than the sum of the thickness of the metal and the dielectric layers $2 t+s$, where $t$ and $s$ are thicknesses of the metal and the dielectric layers, respectively. Notice the propagation direction is perpendicular to the plane of the fishnet with the electric and magnetic fields along the $x$ and $y$ directions, respectively. All retrieved effective parameters are for this particular incident direction and field polarization. 
Consider two configurations, one without gain and one with gain (see inset of Figure 4.19). In the configuration with gain, we have introduced two thin dielectric layers of thickness, $\left(s-h_{g}\right) / 2$, close to the metallic structure, so the gain medium will not be close to the metal, then quenching will be avoided. The dimensions of the fishnet structure $[28,128]$ are chosen such that the magnetic resonance wavelength at $\lambda=2000 \mathrm{~nm}$, which can overlap with the peak of the emission of the gain material. The full width at half maximum (FWHM) of the gain material is $20 \mathrm{THz}$ and the pumping rate, $\Gamma_{\text {pump }}$, changes from 0 to $6.9 \times 10^{8} \mathrm{~s}^{-1}$. In the FDTD calculations, the discrete time and space steps are chosen to be $\Delta t=8.0 \times 10^{-18} \mathrm{~s}$ and $\Delta x=5.0 \times 10^{-9} \mathrm{~m}$.

\subsubsection{Transmission $T$, reflection $R$, and absorption $A$ spectra and retrieval results}
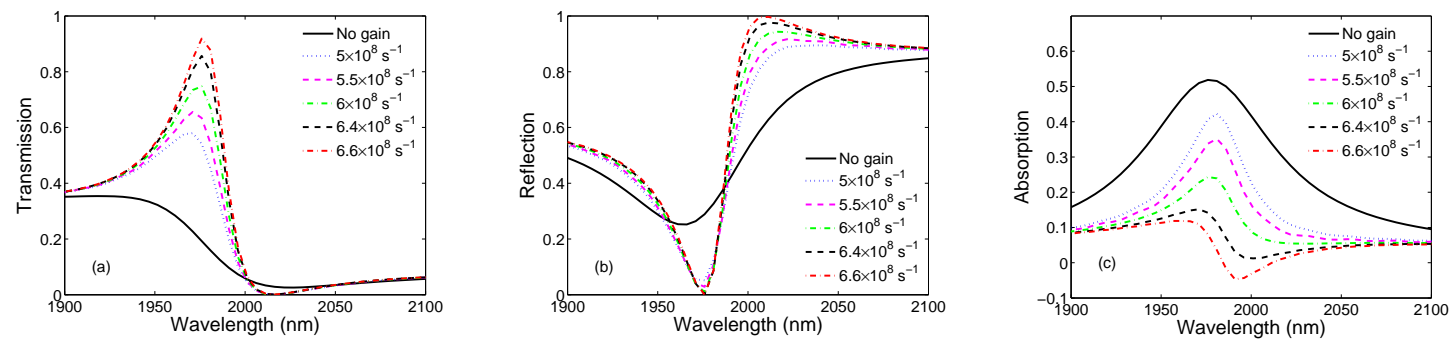

Figure 4.20 The (a) transmission, (b) reflection, and (c) absorption as a function of wavelength for different pumping rates.

In Figure 4.20, we plot the transmission, $T=|t|^{2}$, reflection, $R=|r|^{2}$, and absorption, $A=1-T-R$ versus wavelength for different pumping rates ( $t$ and $r$ are the transmission and reflection amplitudes, respectively). Notice the wavelength dependence of $T$ and $R$ for different pumping rates away from the resonance wavelength, $\lambda=2000 \mathrm{~nm}$, are the same. Below the resonance wavelength, $T$ increases with the pumping rate and above the resonance wavelength, $T$ decreases with the pumping rate. The reflection $R$, below the resonance wavelength, decreases with the pumping rate and above the resonance wavelength, it increases with the pumping rate. Notice in Figure 4.20(a), $T$ without gain has a very weak resonance, and once the pumping rate increases, the transmission clearly shows the resonance behavior. The same can be seen in the experiments that can use gain materials to compensate the losses in fishnet metamaterials. 
In Figure 4.20(c), we plot the absorption, $A$, as a function of wavelength for different pumping rates. Notice, as we increase the pumping rate, the absorption decreases and finally at the pumping rate of $6.6 \times 10^{9} \mathrm{~s}^{-1}$, the gain overcompensates the losses and the absorption becomes negative. In Figure 4.21(a), we plot the retrieved results of the real and imaginary parts of the magnetic permeability, $\mu$, with and without gain, for normal incidence and the particular field polarization in Figure 4.19. As gain increases, the $\operatorname{Re}(\mu)$ becomes steeper at the resonance wavelength and the $\operatorname{Im}(\mu)$ becomes much narrower when increasing the pumping rate and the losses are compensated by the gain material. In Figure 4.21(b), we plot the retrieved results for the effective index of refraction $n$, with and without gain. The $\operatorname{Re}(n)$ becomes more negative after gain is introduced and the $\operatorname{Im}(n)$ also drops significantly close to the resonance. At $\lambda=1976 \mathrm{~nm}$, the $\operatorname{Re}(n)$ changes from -2.25 to -2.82 with a pumping rate of $5.0 \times 10^{8} \mathrm{~s}^{-1}$ and the $\operatorname{Im}(n)$ drops from 1.58 to 0.54 (Fig. 3b). In Figure 4.21(c), we plot the FOM versus the wavelength for different pumping rates. Notice, the FOM becomes very large (of order of $10^{2}$ ) with the pumping rates. Comparing $\operatorname{Im}(n)$ slightly below the resonance at $\lambda=1976 \mathrm{~nm}$, we find the effective extinction coefficient $\alpha=\frac{\omega}{c} \operatorname{Im}(n) \approx 5.0 \times 10^{4} \mathrm{~cm}^{-1}$ without gain and $\alpha \approx 1.7 \times 10^{4} \mathrm{~cm}^{-1}$ with gain $\left(\Gamma_{\text {pump }}=5.0 \times 10^{8} \mathrm{~s}^{-1}\right)$. Hence, an effective amplification of $\alpha=-3.3 \times 10^{4} \mathrm{~cm}^{-1}$. This is much larger (of the order of 30) than the expected amplification

$\alpha \approx-1.3 \times 10^{3} \mathrm{~cm}^{-1}$ for the gain material at the given pumping rate of $5.0 \times 10^{8} \mathrm{~s}^{-1}$. The difference can be explained by the field enhancement in the fishnet metamaterial.

\subsubsection{Kramers-Kronig relations for metamaterials with gain}

There are theoretical debates [129-133] if it is possible to obtain low loss metamaterials with negative refractive index, $n$. They have used the Kramers-Kronig (KK) relations and we would like to verify that Kramers-Kronig relations work with and without gain. In addition, we need to compare the numerically-retrieved effective permeability, $\mu$, shown in Figure 4.21(a), with the calculation of $\mu$, based on the Kramers-Kronig relations. In Figure 4.22(a), we plot the real and imaginary parts of the effective permeability, $\mu$, and the results from the Kramers-Kronig relations for the pumping rate of $\Gamma_{\text {pump }}=5.0 \times 10^{8} \mathrm{~s}^{-1}$. The excellent agreement between the 

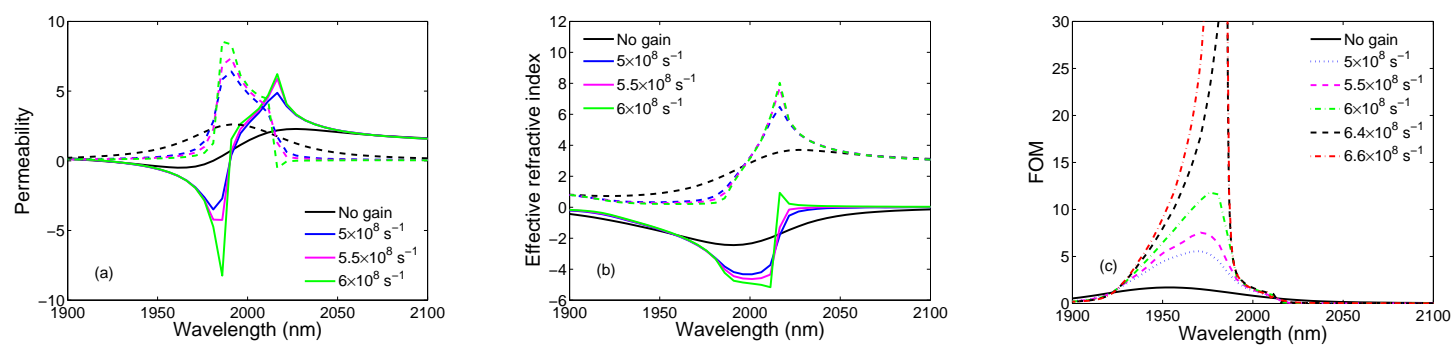

Figure 4.21 The retrieved results for the real (solid lines) and the imaginary (dashed lines) parts of (a) the effective permeability, $\mu$, and (b) the corresponding effective index of refraction, $n$, without and with gain for different pumping rates. (c) The figure-of-merit (FOM) as a function of wavelength for different pumping rates. The gain bandwidth is $20 \mathrm{THz}$.

results obtained from the standard retrieval method [126] and the Kramers-Kronig approach verifies KK relations work for metamaterials coupled with gain too. KK relations are valid for passive materials and the deduction of KK relations is based on the assumption that the response function of materials is analytical in the upper complex plane of frequency. For strong active materials, there are poles in the upper plane and one must modify the KK relations by reversing the signs of KK relations [133]. In Figure 4.22(b), we plot the $\operatorname{Re}(\mu)$ and $\operatorname{Im}(\mu)$ obtained by the retrieval method and by the modified KK relations for the pumping rate $\Gamma_{\text {pump }}=6.9 \times 10^{8} \mathrm{~s}^{-1}$. For this pumping rate the $\operatorname{Im}(\mu)$ becomes negative and we have overcompensated at the resonance frequency of $150 \mathrm{THz}$. As one can see from Figure 4.22(b), KK relations work well for strongly active materials.

\subsubsection{Conclusions}

We have proposed and numerically solved a self-consistent model incorporating gain in the 3D fishnet dispersive metamaterial. We show numerically that one can compensate the losses of the fishnet metamaterial. We have presented results for $T, R$, and $A$ without and with gain for different pumping rates. Once the pumping rate increases, both $T$ and $R$ show a resonance behavior. We have retrieved the effective parameters for different pumping rates and the losses are compensated with gain. Kramers-Kronig relations of the effective parameter 

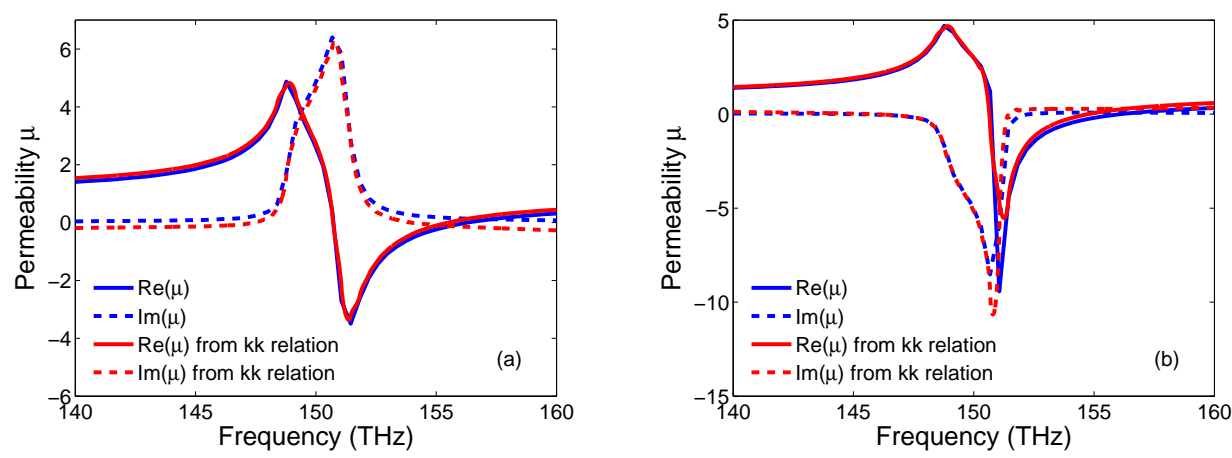

Figure 4.22 The real and imaginary parts of the retrieved effective permeability, $\mu$, and the results from the Kramers-Kronig relations for pumping rates (a) $\Gamma_{\text {pump }}=5.0 \times 10^{8} \mathrm{~s}^{-1}$ (below compensation) and (b) $\Gamma_{\text {pump }}=6.9 \times 10^{8} \mathrm{~s}^{-1}$ (overcompensated).

are in excellent agreement with the retrieved results with gain. The figure-of-merit (FOM) with gain increases dramatically and the pumping rate needed to compensate the loss is much smaller than the bulk gain. This aspect is due to the strong local-field enhancement inside the fishnet structure.

\subsection{Overcoming Losses with Gain in 3D SRR Metamaterials}

\subsubsection{Geometric dimensions of 3D SRR metamaterials}

Except the fishnet structure, we also have studied the loss compensation of 3D SRR metamaterials with gain. Our collaborators in Karlsruhe are doing experiments to compensate the losses of such kind of metamaterial structures with semiconductor quantum wells. Figure 4.23(a) shows a layered structure where the SRR is fabricated on a GaAs-gain-GaAs sandwich substrate. The GaAs layer between the SRR and gain is introduced to avoid the quenching effect. The incident wave propagates along the $y$ direction parallel to the SRR plane and has the magnetic field perpendicular to that plane. The unit cell size along the propagation direction is $a$. In $z$ direction, the unit cell size is $h$, which is larger than $h_{1}+h_{2}+h_{3}+h_{s}$, where $h_{1}, h_{2}, h_{3}$ and $h_{s}$ are the thicknesses of the bottom GaAs layer, the gain layer, the GaAs spacing layer, and the SRR, respectively. Along the unit cell boundaries in $x$ and $z$ directions, 
periodic boundary conditions are enforced to simulate the infinite periodic structure. All the dimensions are chosen to have the magnetic resonance overlap with the emission frequency of $100 \mathrm{THz}$ of the gain material. For comparison, we also introduce another gain configuration (see Figure 4.23(b)), where the gain is embedded in the gap of the SRR instead of a layer underneath. The dimensions keep the same as Figure 4.23(a). In the FDTD simulations, the discrete time and space steps are chosen to be $\Delta t=2.0 \times 10^{-18} \mathrm{~s}$ and $\Delta x=2.5 \times 10^{-9} \mathrm{~m}$.

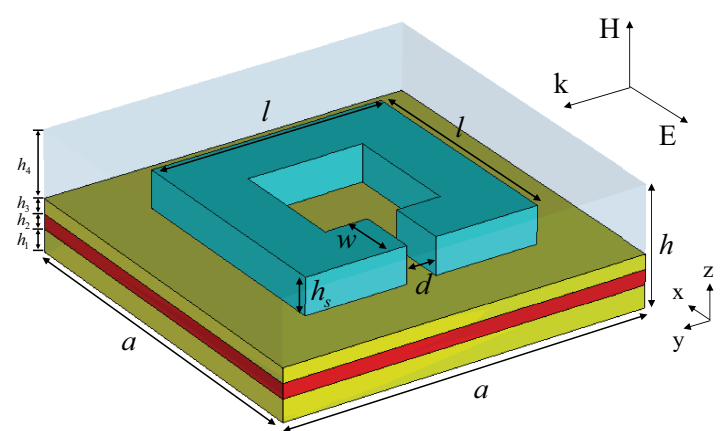

(a)

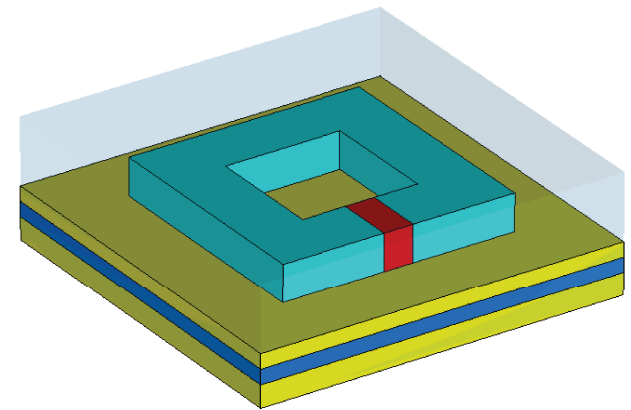

(b)

Figure 4.23 (a) One unit cell for the silver-based SRR structure (light blue) with the gain layer underneath. The dielectric constants $\varepsilon$ for GaAs (yellow) and gain (red) are 11 and 2, respectively. The whole structure is in vacuum background (light gray). The dimensions are $a=250 \mathrm{~nm}, l=160 \mathrm{~nm}, h=80 \mathrm{~nm}$, $h_{1}=15 \mathrm{~nm}, h_{2}=h_{3}=10 \mathrm{~nm}, h_{4}=45 \mathrm{~nm}, h_{s}=25 \mathrm{~nm}$, $w=40 \mathrm{~nm}$ and $d=20 \mathrm{~nm}$. (b) same as (a) except the gain is embedded in the SRR gap with $\varepsilon=1$ and the gain layer in (a) is replaced by a dielectric layer $(\varepsilon=2)$ (blue).

\subsubsection{Numerical simulations and discussions}

In this section, we apply three different pumping schemes on the gain layer shown in Figure 4.23(a): (1) the gain is isotropic and pumped with a homogeneous pumping rate, (2) the gain is isotropic but has a shadow cast by the SRR where the gain is off, elsewhere it has a homogeneous pumping and (3) the gain is anisotropic, i.e., it is only pumped in one selected direction. The linewidths of the magnetic resonances for different pumping rates are investigated to see if the gain can effectively reduce the magnetic losses. Simulation results show that the homogeneously pumped isotropic gain can significantly reduce the losses, while 
the other two schemes do have contributions to loss compensation but are not so efficient. The electric field distributions in the cross-section of the gain layer ( $x y$ plane) are plotted to explain the big difference in loss compensation among the three different gain pumping schemes. Since the GaAs layer $(\varepsilon=11)$ has much higher dielectric constant than the gain $(\varepsilon=2)$ in this structure, the electric field may be bounded in the spatial vicinity of the high dielectric layer, then the interaction between the gain material and the SRR may be affected. We have also done simulations for different gain background dielectric constants to see how it affects the loss compensation. Results show that for anisotropic gain, the gain dielectric background does not significantly change the loss compensation.

\subsubsection{Isotropic gain}

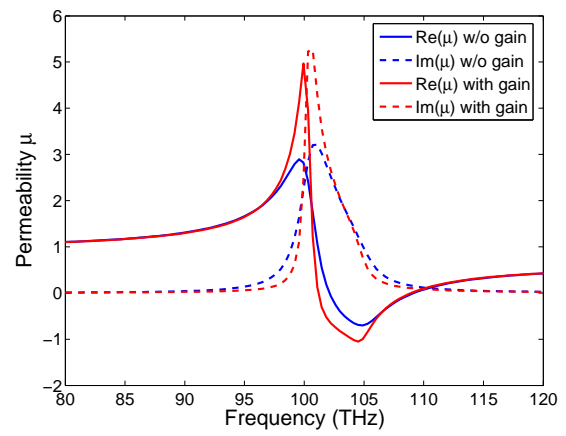

Figure 4.24 The retrieved results for the real and imaginary parts of the effective permeability $\mu$, with and without gain, for the gain configuration shown in Figure 4.23(a). For the case with gain, the pumping rate $\Gamma_{\text {pump }}=1.5 \times 10^{9} \mathrm{~s}^{-1}$.

We first let a wide band Gaussian pulse of a given amplitude go through one layer of the SRR structure shown in Figure 4.23(a) and calculate the transmission $T$, the reflection $R$, and the absorption $A=1-T-R$, as a function of frequency in the propagation direction. With the introduction of gain, the absorption near the resonance frequency $f=100 \mathrm{THz}$ decreases and the transmission increases. To investigate the loss reduction of the magnetic resonators, we plot the retrieved effective permeabilities $\mu$ without gain and with gain by inverting the scattering amplitudes $[126,134]$ in Figure 4.24 . One can see the gain undamps the magnetic 


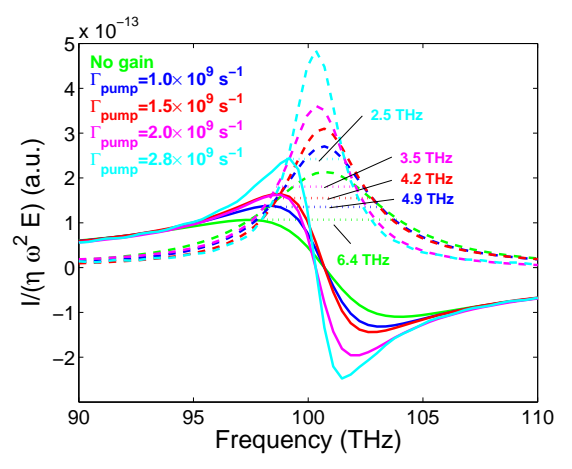

(a)

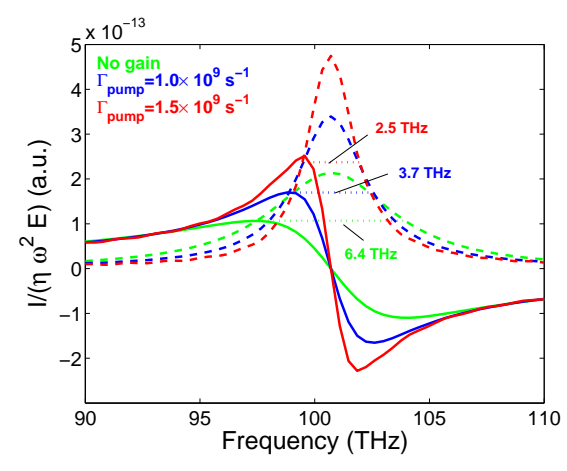

(b)

Figure 4.25 The real (solid) and imaginary (dashed) parts of $I /\left(\eta \omega^{2} E\right)$ as a function of frequency for different pumping rates. (a) For the structure with a gain layer below the SRR, shown in Figure 4.23(a). (b) For the structure with the gain in the SRR gap as shown in Figure 4.23(b). Notice that the resonance is getting stronger and narrower as the pumping rate increases.

resonance of the SRR and the resonant effective permeability $\mu$ of the SRR becomes much stronger and narrower compared to the case without gain. However, due to the periodicity effect, the strong magnetic resonance in Figure 4.24 gets misshapened [134], which causes the difficulty in measuring the linewidth of the magnetic resonance. The periodicity effect itself is inherent in the retrieval procedure. To distinguish the magnetic resonance of the SRR from the periodicity effect of the structure, we directly calculate the resonant current (i.e., the magnetic moment) flowing around the split ring, so we do not need to go through the retrieval procedure. Consider the SRR as a simple LCR circuit model, we can have the following equation,

$$
L \frac{d I}{d t}+\frac{\int I d t}{C}+I R=\varepsilon_{\mathrm{emf}}
$$

where $L, C$ and $R$ are the effective inductance, capacitance and resistance of the SRR, respectively, and $I$ is the current flowing in the SRR and $\varepsilon_{\mathrm{emf}}$ is the induced electromotive force. From Faraday's law, $\varepsilon_{\mathrm{emf}}=-d \Phi / d t=i A \mu_{0} \omega H=i A \frac{\omega}{c} E$. ( $\Phi$ is the magnetic flux through the $\mathrm{SRR}, A$ is the area enclosed by SRR, and $c$ is the speed of light in vacuum.) Then we can 
obtain the expression with Lorentz resonance shape,

$$
\frac{I}{\eta \omega^{2} E}=-\frac{1}{\omega^{2}-\omega_{0}^{2}+i \gamma \omega}
$$

where $\eta, \omega_{0}$, and $\gamma$ are $A /(c L), 1 / \sqrt{L C}$, and $R / L$, respectively. The detailed results are plotted in Figure 4.25(a) for the structure shown in Figure 4.23(a). One can see the current resonances have very nice Lorentz line shapes. As the pumping rate increases, the resonance is stronger and narrower. The full width at half maximum (FWHM) reaches $2.5 \mathrm{THz}$ when the pumping rate $\Gamma_{\text {pump }}=2.8 \times 10^{9} \mathrm{~s}^{-1}$, which is a significant loss reduction compared with the FWHM without gain $(\mathrm{FWHM}=6.4 \mathrm{THz})$. So the gain compensates the losses. In addition, we also calculate $I /\left(\eta \omega^{2} E\right)$ vs. frequency curves for the gain configuration shown in Figure 4.23(b) to compare the efficiency of the loss compensation for these two different gain configurations. The results are shown in Figure 4.25(b). One can see the structure with gain in the SRR gap needs less gain (i.e., smaller pumping rate) to reach the same FWHM of the resonance than the case with gain underneath the SRR. For example, the latter structure needs the pumping rate $\Gamma_{\text {pump }}=2.8 \times 10^{9} \mathrm{~s}^{-1}$ to have $\mathrm{FWHM}=2.5 \mathrm{THz}$ while the former only needs the pumping rate $\Gamma_{\text {pump }}=1.5 \times 10^{9} \mathrm{~s}^{-1}$. It is straightforward because of the strong local electric field enhancement in the SRR gap. Though the loss compensation for the structure with a gain layer underneath the SRR is not so efficient as the case with the gain in the SRR gap, the results in Figure 4.25(a) still show that the magnetic losses can be significantly reduced, especially if we push the pumping rate to a high value.

We also have studied the normal incidence case where the direction of propagation is perpendicular to the SRR plane and the electric field is parallel to the gap bearing side of the SRR, such that the electric field can couple to the electric dipole in the gap and induce the magnetic resonance $[135,136]$. We want to see if the losses can be compensated by the gain layer underneath the SRR for the normal incidence. With this incidence direction, the unit cell size in the propagation direction is $h$, which is much smaller than the wavelength $\lambda$, so the resonance is far below the Brillouin zone edge and then there is no periodicity effect. Figure 4.26 plots the retrieved effective permittivity $\varepsilon$, with and without gain. Both of them 


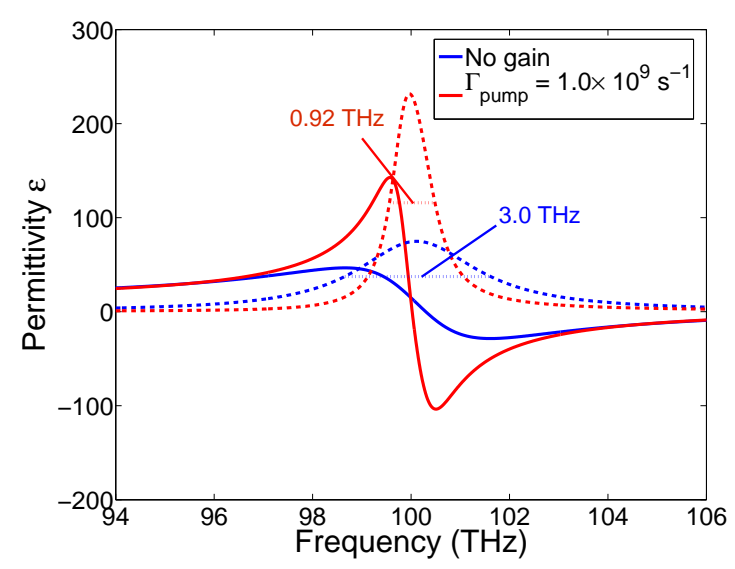

Figure 4.26 The retrieved results for the real and imaginary parts of the effective permittivity $\varepsilon$, with and without gain, for the normal incidence in Figure 4.23(a). For the case with gain, the pumping rate $\Gamma_{\text {pump }}=1.0 \times 10^{9} \mathrm{~s}^{-1}$.

have a very nice Lorentz line shape. Without gain, the resonance is broad and weak, and the FWMH is $3 \mathrm{THz}$. With the introduction of gain, the resonance gets stronger and narrower, and the FWHM reduces to a much smaller value, $0.92 \mathrm{THz}$. So the gain effectively compensates the losses of the SRR for normal incidence.

\subsubsection{Isotropic gain with a shadow of the SRR}

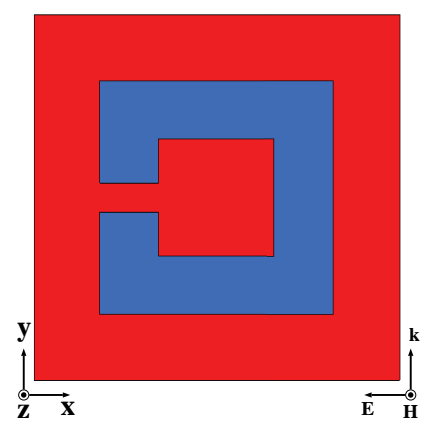

Figure 4.27 Top view of the gain layer in Figure 4.23(a) when a shadow (blue) is cast by the SRR structure. The gain goes away in the shadow, while in other area (red) it is homogeneously pumped.

So far, the gain material in our simulations is pumped by a homogeneous pumping rate 
$\Gamma_{\text {pump. }}$. This is an ideal case. Consider the case in experiments that we incident an external electromagnetic pumping wave on the structure from the top in Figure 4.23(a) to optically pump the electrons from level 0 to level 3 , there is a shadow in the gain layer cast by the SRR structure, where the gain is pumped by a much lower rate. As a simplified model, we turn off the gain in the area which lies directly under the SRR to simply emulate the shadow of the SRR structure, while we still keep a homogeneous pumping rate $\Gamma_{\text {pump }}$ in other gain area (see Figure 4.27). In Figure 4.28(a), we plot $I /\left(\eta \omega^{2} E\right)$ as a function of frequency in this case. Compared with the case without the shadow in the gain layer (Figure 4.25(a)), the resonance gets much weaker and broader $(\mathrm{FWHM}=5.7 \mathrm{THz}$ and $5.4 \mathrm{THz}$ for the pumping rates $\Gamma_{\text {pump }}=1.0 \times 10^{9} \mathrm{~s}^{-1}$ and $1.5 \times 10^{9} \mathrm{~s}^{-1}$, respectively). This shows the gain in the shadow area plays an important part in the loss compensation.

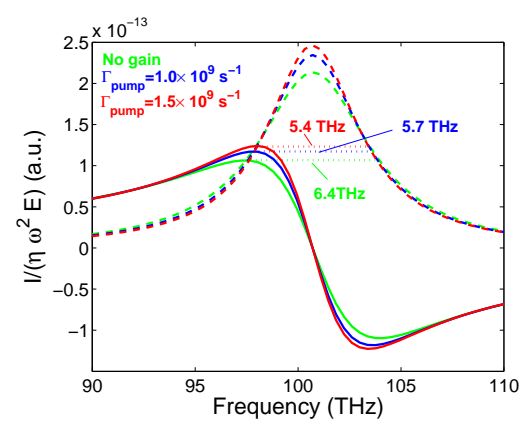

(a)

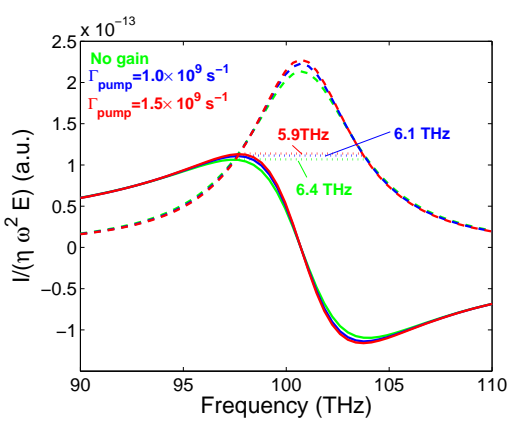

(b)

Figure 4.28 The real (solid) and imaginary (dashed) parts of $I /\left(\eta \omega^{2} E\right)$ as a function of frequency for different pumping rates. (a) For the structure with a shadow in the gain layer cast by the SRR. (b) For the structure where the gain is pumped in $y$ direction only (see the coordinate system in Figure 4.23(a)).

\subsubsection{Anisotropic gain}

The gain taken in our simulations above is the isotropic gain material, which is equally pumped in all directions. The realistic gain, such as semiconductor quantum dots/wells, can be anisotropic, i.e., it can only couple to the external field in a certain direction. In our following simulations we take into considerations one gain material which is only pumped in $y$ direction. 
It means the gain only couples to the electric field in $y$ direction. The corresponding $I /\left(\eta \omega^{2} E\right)$ vs. frequency curves for different pumping rates are plotted in Figure 4.28(b). One can see the resonances are also much broader than the case with homogeneously pumped isotropic gain. So the loss compensation is less efficient.

\subsubsection{Explanation of the differences among the three pumping schemes}

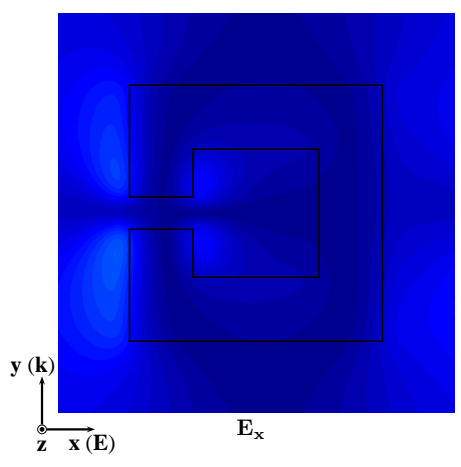

(a)

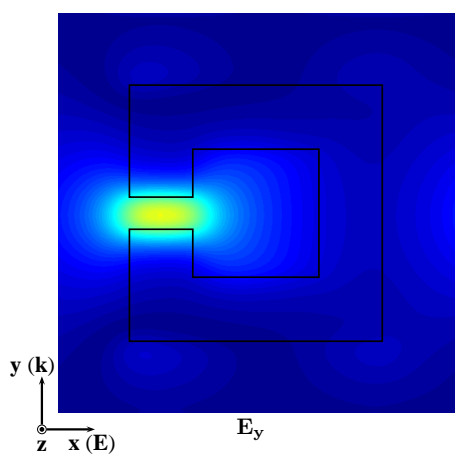

(b)

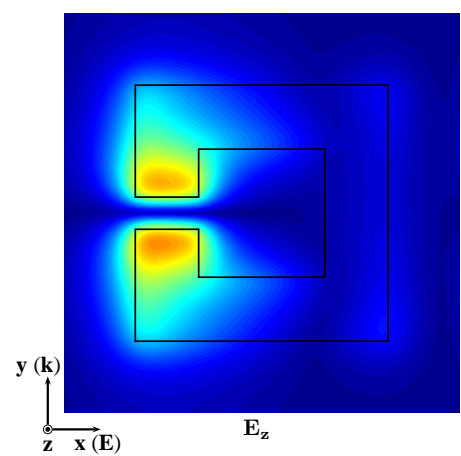

(c)

Figure 4.29 The electric field amplitude distribution at the resonance frequency in the cross-section of the gain layer ( $x y$ plane in Figure 4.23(a)) for different components: (a) $E_{x}$, (b) $E_{y}$ and (c) $E_{z}$. The area enclosed by the black line is the projection of the $\mathrm{SRR}$ in the gain layer. The electric field is calculated without gain.

To see why these three gain pumping schemes are so different in the loss compensation, we have calculated the electric field amplitude distribution in the cross-section of the gain layer ( $x y$ plane in Figure 4.23(a)). The detailed results are plotted in Figures 4.29(a)-4.29(c). One can see the $x$ component of electric field, $E_{x}$, is very weak while the other two components, $E_{y}$ and $E_{z}$, are relatively strong. So we can ignore the gain contribution by $E_{x}$ and focus on the gain from the coupling with $E_{y}$ and $E_{z}$. Notice that $E_{y}$ is bounded in the area right below the SRR gap (Figure 4.29(b)) while $E_{z}$ mainly has a significant value in the projection of the SRR in the gain layer (Figure 4.29(c)). This characteristic of the field amplitude distribution leads to almost no contribution by $E_{z}$ when we have a shadow in the gain layer since there is no gain in that area. Similarly, the gain contribution by $E_{z}$ goes away for the anisotropic gain 
because the gain only couples with the $y$ component of the electric field, $E_{y}$. This fact explains the big difference between the homogeneously pumped isotropic gain case and the other two gain pumping schemes.

\subsubsection{The effect of the dielectric background of gain}

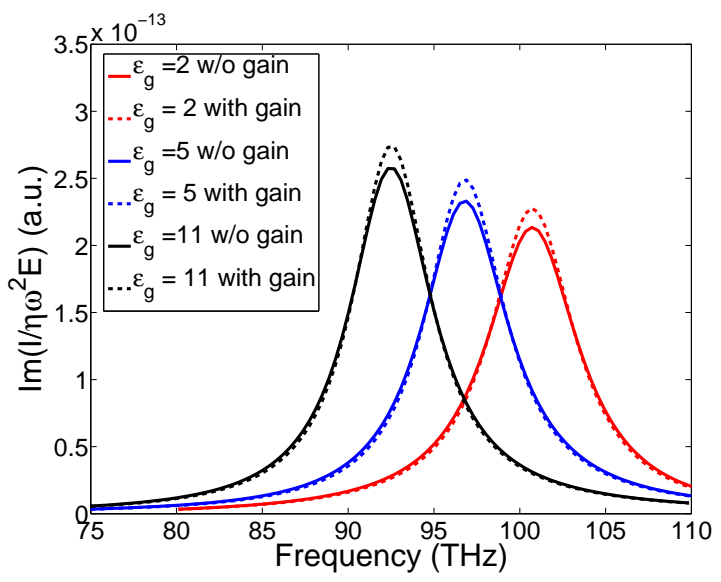

Figure 4.30 The imaginary parts of $I /\left(\eta \omega^{2} E\right)$ as a function of frequency for different background dielectric constants of the gain material, which is only pumped in $y$ direction. For the case with gain, the pumping rate is $\Gamma_{\text {pump }}=1.5 \times 10^{9} \mathrm{~s}^{-1}$. Note the resonance enhancements by the gain are almost the same.

Since there is a high contrast between the dielectric constants of the GaAs $(\varepsilon=11)$ and gain $(\varepsilon=2)$ layers, the electromagnetic fields may be bounded in the high dielectric layer. In this section, we will discuss the effect of the dielectric background of gain on the loss compensation. In Figure 4.30, we plot the detailed results for the imaginary parts of $I /\left(\eta \omega^{2} E\right)$ as a function of frequency, with and without gain, for the background dielectric constants of the gain layer $\varepsilon_{g}=2,5$ and 11. The gain is anisotropic and only couples to the electric field in $y$ direction. We can see the resonance frequency shifts down as the dielectric constant increases. This is expected since the effective capacitance increases with the increment of the dielectric constant. To effectively compensate the losses, we scale the emission frequency to overlap with the corresponding resonance frequencies and then pump with the same rate $\Gamma_{\text {pump }}=1.5 \times 10^{9} \mathrm{~s}^{-1}$. We can see from Figure 4.30 that the resonance enhancements are almost the same for different 
background dielectric constants of the gain.
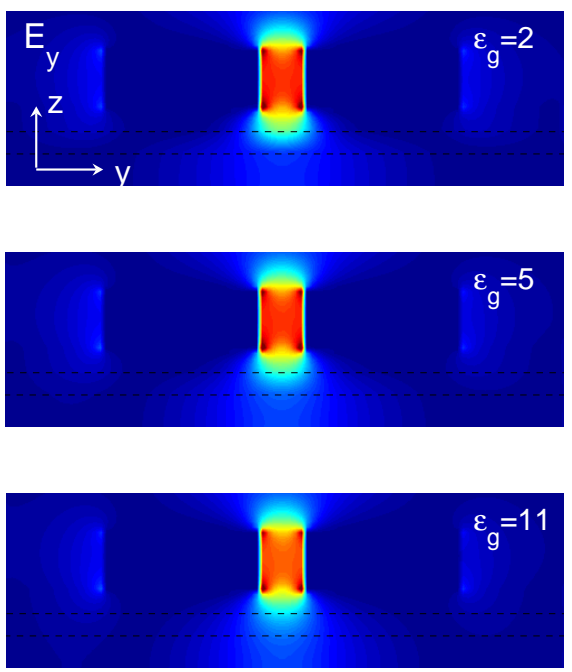

(a)
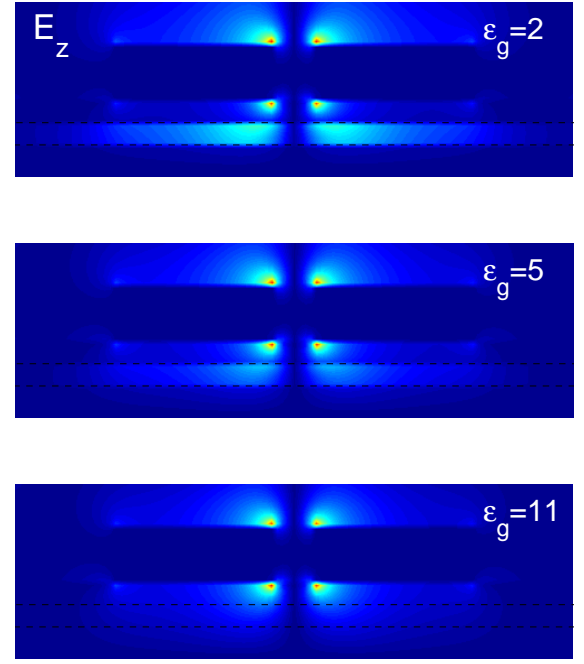

(b)

Figure 4.31 The electric field amplitude distributions for different background dielectric constants of the gain material $\left(\varepsilon_{g}=2,5\right.$ and 11) at their corresponding resonance frequencies, in a $y z$ plane crossing the middle of the gap bearing side of the SRR. (a) $E_{y}$ and (b) $E_{z}$. The area enclosed by the dashed black line indicates the position of the gain layer. The electric field is calculated without gain.

To explain this, we plot the electric field amplitude distributions in a plane crossing the middle of the gap bearing side of the SRR in Figure 4.31, for $\varepsilon_{g}=2,5$ and 11, respectively. The $E_{x}$ component is ignored since it is very weak as show in Figure 4.29(a). From Figure 4.31(a), we can see the field amplitude distribution of $E_{y}$, the only component which couples to the gain, does not change much in the gain layer as the gain background dielectric constant changes. Although there is a bounding effect on the fields, the $y$ component of the electric field, $E_{y}$, does not substantially decay in such a very narrow gain layer $(10 \mathrm{~nm})$ neighboring to the high dielectric GaAs layer. The main change in the electric field is the $z$ component of the electric field, $E_{z}$, decreases in the gain layer as the gain background dielectric constant, $\varepsilon_{g}$, increases, as shown in Figure 4.31(b). This is due to the continuity of the normal component of the electric displacement across the interface if there is no free charge accumulation. Hence the normal component of the electric field is inversely proportional to the dielectric constant. The 
change of $E_{z}$ does not affect the loss compensation due to no coupling between the gain and $E_{z}$. If the gain can couple to $E_{z}$, such as the isotropic gain, the background dielectric constant of the gain will significantly affect the loss compensation.

\subsubsection{Conclusions}

We have numerically studied the loss compensation of the silver-based SRR structure with a gain layer underneath. Numerical results show that the losses of the SRR can be effectively compensated by the gain layer for both the parallel and perpendicular incidences. For the perpendicular incidence, the electric field of the incident wave is parallel to the gap bearing side of the SRR. Three different gain pumping schemes are applied in the simulations and the efficiencies of their corresponding loss compensations are studied by investigating the linewidth of the magnetic resonance. To avoid the distortion of the Lorentz resonant lineshape in the retrieved permeability, $\mu$, due to the periodicity effect, the resonant current in the SRR is calculated and then the linewidth of the magnetic resonance is obtained. The homogeneously pumped isotropic gain can significantly reduce the magnetic losses, though it is less efficient in the loss compensation compared to the case with the gain in the SRR gap. The other two schemes, (1) a shadow is cast in the gain layer by the SRR and the gain goes away over that region and (2) the gain is anisotropic and only couples to the electric field in $y$ direction, are much less efficient in the loss compensation compared to the isotropic gain case, due to no interactions between $E_{z}$ and the gain in these two schemes. We have also studied the effect of the background dielectric of gain. As the gain background dielectric constant increases, the electric component parallel to the interface does not change much in a very narrow layer neighboring the high dielectric layer, instead, the normal component significantly changes due to the continuity of the normal component of the electric displacement across the interface. The change in the electric field will only affect the loss compensation for the gain which couples to the electric field in the normal direction. For the gain pumped in the parallel direction only, the background dielectric of the gain does not make much difference. 


\section{CHAPTER 5. SUMMARY}

The field of metamaterials has made great progress towards real applications in the last decade. However, the envisioned applications are limited by the huge intrinsic losses of metamaterials. Reducing the losses of metamaterials has become a big challenge in the research of metamaterials. This thesis shows my theoretical studies in the loss reduction. There are two approaches presented for reducing the losses of metamaterials.

One approach is to avoid the electric and magnetic resonances and hence reduce the losses. By this approach, 3D metallic nanowire media with different structures are designed at the optical frequency region and it is numerically demonstrated that they can be homogeneous effective indefinite anisotropic media by showing their dispersion relations are hyperbolic. For a finite slab, a nice fitting procedure is exploited to obtain the dispersion relations, from which the effective permittivities are retrieved. The pseudo focusing for the real 3D wire medium agrees very well with the homogeneous medium having the effective permittivity tensor of the wire medium. Studies also show that in the long wavelength limit, the hyperbolic dispersion relation of the $3 \mathrm{D}$ wire medium can be valid even for evanescent modes, which may have important applications in super-resolution.

Another approach is to incorporate the gain in the metamaterial system. It is the most promising and also more generic way to reduce the losses of metamaterials because for other methods, such as geometry optimization, the loss is still orders of magnitude too large for the envisioned applications. The gain material is modeled by a generic semi-classical four-level atomic system and a computational scheme is presented, which allows for a self-consistent treatment of a dispersive metallic photonic metamaterial coupled to a gain material incorporated into the nanostructure. Simulations are done for the loss compensations of 1D-3D 
metamaterials with gain and results show the losses of the dispersive metamaterial structures presented in this thesis can be compensated by gain.

In 1D case, the lasing and nonlinear behaviors are studied for gain slabs and a 1D metamaterial system consisted of layers of negative index material and gain material. A critical pumping rate exists for compensating the loss of the metamaterial. For a fixed pumping rate, there is a relatively wide range of signal amplitudes for which the loss compensated metamaterial still behaves linearly. At higher amplitudes, nonlinearities arise due to gain depletion. It is necessary to have self-consistent calculations to determine the signal range where we can expect a linear response. Further, if we have strong signals, so that we are in nonlinear regime, or we want to study lasing, the self-consistent calculation is needed.

In 2D case, simulations are done for a lattice of resonant square cylinders embedded in layers of gain material and split ring resonators with gain material embedded into the gaps. Transmission, reflection, and absorption data as well as the retrieved effective parameters are presented. It is numerically shown the magnetic losses of the SRR can be compensated by the electric gain and the effective gain amplification coefficient of the combined system can be much larger than the bulk gain due to the strong local field enhancement.

In 3D case, a fishnet structure and an array of SRRs are studied. The fishnet structure has a magnetic resonance at the wavelength of $2000 \mathrm{~nm}$. It is numerically shown that one can compensate the losses of the fishnet metamaterial by the gain. Simulation results for the transmission, $T$, reflection, $R$, and absorption, $A$, are presented. With the introduction of gain, $T$ and $R$ show a strong resonance behavior. The effective index of refraction, $n$, and permeability, $\mu$, are presented for different pumping rates and clearly show the losses are compensated with gain and the magnetic resonance becomes stronger with the gain. The figureof-merit $(\mathrm{FOM}) \mathrm{FOM}=|\operatorname{Re}(n) / \operatorname{Im}(n)|$ is calculated from the retrieved $n$. It dramatically increases with gain. Due to the strong local-field enhancement, the pumping rate needed to compensate the losses is much smaller than the bulk gain. Kramers-Kronig relations are verified by comparing the numerically retrieved permeability, $\mu$, with the calculation of $\mu$, based on the Kramers-Kronig relations. They can work very well for strongly active materials. The array of 
SRRs are fabricated on a GaAs-gain-GaAs sandwich substrate and has the magnetic resonance at $100 \mathrm{THz}$. Three different pumping schemes are applied on the gain: (1) isotropic gain, (2) isotropic gain with a shadow cast by the SRR where the gain goes away, and (3) anisotropic gain which only couples to the electric field component parallel to the gap bearing side of the SRR. For the isotropic gain, the gain can effectively compensate the magnetic losses of the SRR for both the parallel incidence and the perpendicular incidence which electrically couples to the SRR. To avoid the distortion of the Lorentz lineshape in the retrieved permeability, $\mu$, due to the periodicity effect, the resonant current in the SRR is calculated. Loss compensation is investigated by comparing the linewiths of the current resonances for different pumping rates. The isotropic gain with a shadow and the anisotropic gain have similar results and are not so effective in the loss compensation as the isotropic gain, though both of them do compensate the losses of the SRR. This is due to no interactions between the gain and the electric component normal to the SRR plane in these two schemes. The effect of the background dielectric of gain is also studied. The background dielectric of gain mainly affects the electric field normal to the SRR plane, so it does not affect the loss compensation of the anisotropic gain, but does affect the loss compensation of the isotropic gain. 


\section{APPENDIX A. THE 3D FDTD UPDATE ALGORITHM}

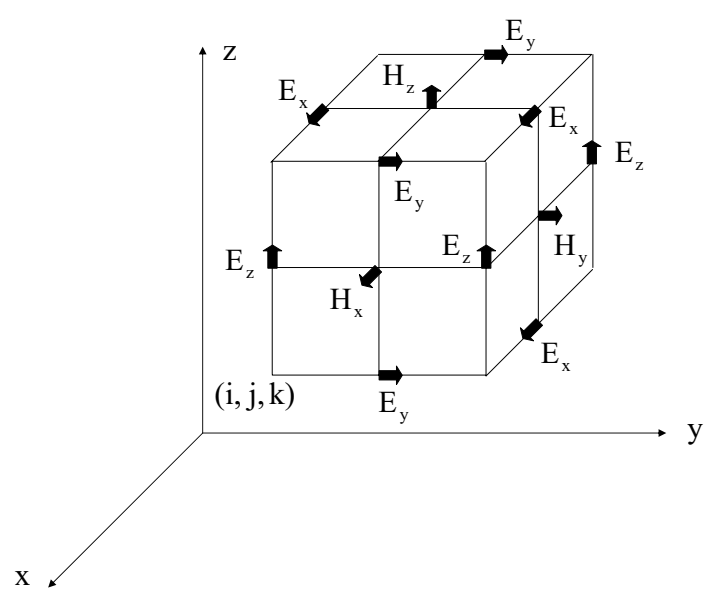

Figure A.1 Schematic of the Yee cell used in discretizing the space.

The Maxwell's equations are spatially discretized on the Yee cell shown in Figure A.1, where the magnetic field dual grid diagonally shifts a vector $(\Delta x / 2, \Delta y / 2, \Delta z / 2)$ from the electric field grid. Here, $\Delta x, \Delta y$, and $\Delta z$ are the lattice space increments in the $x, y$, and $z$ coordinate directions, respectively. Based on this spatial discretization, we show in this appendix the detailed 3D FDTD update algorithm for a system composed of lossy dielectrics, Drude metals and gain materials. To simulate the extension to infinity, the two ends perpendicular to $y$ direction are terminated by PML absorbing boundary conditions. On the boundaries perpendicular to $x$ and $z$ directions, periodic boundary conditions are enforced. To apply the $\mathrm{TF} / \mathrm{SF}$ technique to generate a normal incidence plane wave, we choose two interfaces perpendicular to $y$ direction $j=j_{L}$ and $j=j_{R}$ to connect the total and scattered fields, and they are aligned with the electric field components $E_{x}$ and $E_{z}$. We denote a space point in a uniform, rectangular lattice as $(i, j, k)=(i \Delta x, j \Delta y, k \Delta z)$. Further, we denote any function $u$ of space 
and time at a discrete point in the grid and time as

$$
u^{l}(i, j, k)=u(i \Delta x, j \Delta y, k \Delta z, l \Delta t) .
$$

The fields are updated in the following order:

1. Update the magnetic field components $H_{x}, H_{y}$, and $H_{z}$

In the lossy dielectric, Drude metal and gain material regions,

$$
\begin{aligned}
H_{x}^{l+1 / 2}\left(i, j+\frac{1}{2}, k+\frac{1}{2}\right)= & H_{x}^{l-1 / 2}\left(i, j+\frac{1}{2}, k+\frac{1}{2}\right) \\
& +\frac{\Delta t}{\mu}\left(\frac{E_{y}^{l}\left(i, j+\frac{1}{2}, k+1\right)-E_{y}^{l}\left(i, j+\frac{1}{2}, k\right)}{\Delta z}\right. \\
& \left.-\frac{E_{z}^{l}\left(i, j+1, k+\frac{1}{2}\right)-E_{z}^{l}\left(i, j, k+\frac{1}{2}\right)}{\Delta y}\right), \\
H_{y}^{l+1 / 2}\left(i+\frac{1}{2}, j, k+\frac{1}{2}\right)= & H_{y}^{l-1 / 2}\left(i+\frac{1}{2}, j, k+\frac{1}{2}\right) \\
& +\frac{\Delta t}{\mu}\left(\frac{E_{z}^{l}\left(i+1, j, k+\frac{1}{2}\right)-E_{z}^{l}\left(i, j, k+\frac{1}{2}\right)}{\Delta x}\right. \\
& \left.-\frac{E_{x}^{l}\left(i+\frac{1}{2}, j, k+1\right)-E_{x}^{l}\left(i+\frac{1}{2}, j, k\right)}{\Delta z}\right), \\
& +\frac{\Delta t}{\mu}\left(\frac{E_{x}^{l}\left(i+\frac{1}{2}, j+1, k\right)-E_{x}^{l}\left(i+\frac{1}{2}, j, k\right)}{\Delta y}\right. \\
H_{z}^{l+1 / 2}\left(i+\frac{1}{2}, j+\frac{1}{2}, k\right)= & H_{z}^{l-1 / 2}\left(i+\frac{1}{2}, j+\frac{1}{2}, k\right) \\
& \left.-\frac{E_{y}^{l}\left(i+1, j+\frac{1}{2}, k\right)-E_{y}^{l}\left(i, j+\frac{1}{2}, k\right)}{\Delta x}\right) .
\end{aligned}
$$

In the PML region, where the matching condition $\sigma^{*} / \mu=\sigma / \varepsilon$ has been used,

$$
\begin{aligned}
H_{x y}^{l+1 / 2}\left(i, j+\frac{1}{2}, k+\frac{1}{2}\right)= & \frac{1-\sigma_{y} \Delta t / 2 \varepsilon}{1+\sigma_{y} \Delta t / 2 \varepsilon} H_{x y}^{l-1 / 2}\left(i, j+\frac{1}{2}, k+\frac{1}{2}\right)-\frac{\Delta t / \Delta y \mu_{0}}{1+\sigma_{y} \Delta t / 2 \varepsilon} \\
& \cdot\left[E_{z}^{l}\left(i, j+1, k+\frac{1}{2}\right)-E_{z}^{l}\left(i, j, k+\frac{1}{2}\right)\right],
\end{aligned}
$$




$$
\begin{aligned}
& H_{x z}^{l+1 / 2}\left(i, j+\frac{1}{2}, k+\frac{1}{2}\right)=\frac{1-\sigma_{z} \Delta t / 2 \varepsilon}{1+\sigma_{z} \Delta t / 2 \varepsilon} H_{x z}^{l-1 / 2}\left(i, j+\frac{1}{2}, k+\frac{1}{2}\right)+\frac{\Delta t / \Delta z \mu}{1+\sigma_{z} \Delta t / 2 \varepsilon} \\
& \cdot\left[E_{y}^{l}\left(i, j+\frac{1}{2}, k+1\right)-E_{y}^{l}\left(i, j+\frac{1}{2}, k\right)\right] \text {, } \\
& H_{x}^{l+1 / 2}\left(i, j+\frac{1}{2}, k+\frac{1}{2}\right)=H_{x y}^{l+1 / 2}\left(i, j+\frac{1}{2}, k+\frac{1}{2}\right)+H_{x z}^{l+1 / 2}\left(i, j+\frac{1}{2}, k+\frac{1}{2}\right) . \\
& H_{y z}^{l+1 / 2}\left(i+\frac{1}{2}, j, k+\frac{1}{2}\right)=\frac{1-\sigma_{z} \Delta t / 2 \varepsilon}{1+\sigma_{z} \Delta t / 2 \varepsilon} H_{y z}^{l-1 / 2}\left(i+\frac{1}{2}, j, k+\frac{1}{2}\right)-\frac{\Delta t / \Delta z \mu}{1+\sigma_{z} \Delta t / 2 \varepsilon} \\
& \cdot\left[E_{x}^{l}\left(i+\frac{1}{2}, j, k+1\right)-E_{x}^{l}\left(i+\frac{1}{2}, j, k\right)\right] \text {, } \\
& H_{y x}^{l+1 / 2}\left(i+\frac{1}{2}, j, k+\frac{1}{2}\right)=\frac{1-\sigma_{x} \Delta t / 2 \varepsilon}{1+\sigma_{x} \Delta t / 2 \varepsilon} H_{y x}^{l-1 / 2}\left(i+\frac{1}{2}, j, k+\frac{1}{2}\right)+\frac{\Delta t / \Delta x \mu}{1+\sigma_{x} \Delta t / 2 \varepsilon} \\
& \cdot\left[E_{z}^{l}\left(i+1, j, k+\frac{1}{2}\right)-E_{z}^{l}\left(i, j, k+\frac{1}{2}\right)\right] \text {, } \\
& H_{y}^{l+1 / 2}\left(i+\frac{1}{2}, j, k+\frac{1}{2}\right)=H_{y z}^{l+1 / 2}\left(i+\frac{1}{2}, j, k+\frac{1}{2}\right)+H_{y x}^{l+1 / 2}\left(i+\frac{1}{2}, j, k+\frac{1}{2}\right) . \\
& H_{z x}^{l+1 / 2}\left(i+\frac{1}{2}, j+\frac{1}{2}, k\right)=\frac{1-\sigma_{x} \Delta t / 2 \varepsilon}{1+\sigma_{x} \Delta t / 2 \varepsilon} H_{z x}^{l-1 / 2}\left(i+\frac{1}{2}, j+\frac{1}{2}, k\right)-\frac{\Delta t / \Delta x \mu}{1+\sigma_{x} \Delta t / 2 \varepsilon} \\
& \cdot\left[E_{y}^{l}\left(i+1, j+\frac{1}{2}, k\right)-E_{y}^{l}\left(i, j+\frac{1}{2}, k\right)\right] \text {, } \\
& H_{z y}^{l+1 / 2}\left(i+\frac{1}{2}, j+\frac{1}{2}, k\right)=\frac{1-\sigma_{y} \Delta t / 2 \varepsilon}{1+\sigma_{y} \Delta t / 2 \varepsilon} H_{z y}^{l-1 / 2}\left(i+\frac{1}{2}, j+\frac{1}{2}, k\right)+\frac{\Delta t / \Delta y \mu}{1+\sigma_{y} \Delta t / 2 \varepsilon} \\
& \cdot\left[E_{x}^{l}\left(i+\frac{1}{2}, j+1, k\right)-E_{x}^{l}\left(i+\frac{1}{2}, j, k\right)\right] \text {, } \\
& H_{z}^{l+1 / 2}\left(i+\frac{1}{2}, j+\frac{1}{2}, k\right)=H_{z x}^{l+1 / 2}\left(i+\frac{1}{2}, j+\frac{1}{2}, k\right)+H_{z y}^{l+1 / 2}\left(i+\frac{1}{2}, j+\frac{1}{2}, k\right) .
\end{aligned}
$$

2. Apply the TF/SF technique, and make corrections for $H_{x}$ and $H_{z}$

First we define $E_{\text {inc }}$ is oriented with an angle $\theta\left(0^{0} \leq \theta<180^{\circ}\right)$ relative to $+\mathrm{z}$ axis as shown in Figure A.2. And we have the source position at $j=0$ in the source grid and then have the interfaces $j=j_{L}$ and $j=j_{R}$ coincide with $E_{\text {inc }}\left(j_{L}\right)$ and $E_{\text {inc }}\left(j_{R}\right)$, respectively. The incident 
field components needed to implement the correction formula are as follows,

$$
\begin{aligned}
& E_{z, \text { inc }}^{l}\left(i, j_{L}, k+\frac{1}{2}\right)=E_{\text {inc }}^{l}\left(j_{L}\right) \cos \theta, \\
& E_{z, \text { inc }}^{l}\left(i, j_{R}, k+\frac{1}{2}\right)=E_{\text {inc }}^{l}\left(j_{R}\right) \cos \theta, \\
& E_{x, \text { inc }}^{l}\left(i+\frac{1}{2}, j_{L}, k\right)=-E_{\text {inc }}^{l}\left(j_{L}\right) \sin \theta, \\
& E_{x, \text { inc }}^{l}\left(i+\frac{1}{2}, j_{R}, k\right)=-E_{\text {inc }}^{l}\left(j_{R}\right) \sin \theta
\end{aligned}
$$

2a. Make $H_{x}$ corrections

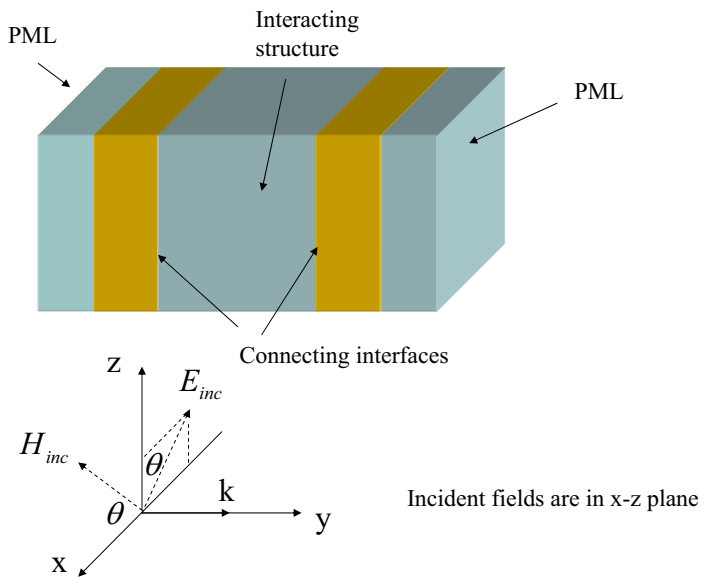

Figure A.2 Schematic of the 3D FDTD computation space and the polarization of the incident fields.

At the left interface $j=j_{L}$,

$$
H_{x}^{l+1 / 2}\left(i, j_{L}-\frac{1}{2}, k+\frac{1}{2}\right)=\left\{H_{x}^{l+1 / 2}\left(i, j_{L}-\frac{1}{2}, k+\frac{1}{2}\right)\right\}+\frac{\Delta t}{\mu_{0} \Delta y} E_{z, \text { inc }}^{l}\left(i, j_{L}, k+\frac{1}{2}\right) .
$$

At the right interface $j=j_{R}$,

$$
H_{x}^{l+1 / 2}\left(i, j_{R}+\frac{1}{2}, k+\frac{1}{2}\right)=\left\{H_{x}^{l+1 / 2}\left(i, j_{R}+\frac{1}{2}, k+\frac{1}{2}\right)\right\}-\frac{\Delta t}{\mu_{0} \Delta y} E_{z, \text { inc }}^{l}\left(i, j_{R}, k+\frac{1}{2}\right) .
$$

2b. Make $H_{z}$ corrections 
At the left interface $j=j_{L}$,

$$
H_{z}^{l+1 / 2}\left(i+\frac{1}{2}, j_{L}-\frac{1}{2}, k\right)=\left\{H_{z}^{l+1 / 2}\left(i+\frac{1}{2}, j_{L}-\frac{1}{2}, k\right)\right\}-\frac{\Delta t}{\mu_{0} \Delta y} E_{x, \text { inc }}^{l}\left(i+\frac{1}{2}, j_{L}, k\right) .
$$

At the right interface $j=j_{R}$,

$$
H_{z}^{l+1 / 2}\left(i+\frac{1}{2}, j_{R}+\frac{1}{2}, k\right)=\left\{H_{z}^{l+1 / 2}\left(i+\frac{1}{2}, j_{R}+\frac{1}{2}, k\right)\right\}+\frac{\Delta t}{\mu_{0} \Delta y} E_{x, \text { inc }}^{l}\left(i+\frac{1}{2}, j_{R}, k\right) .
$$

3. Update $H_{\text {inc }}$ in the source grid generating look-up table

The source grid is terminated with a $1 \mathrm{D}$ PML and $\sigma=0$ outside the PML region.

$$
\begin{aligned}
H_{\mathrm{inc}}^{l+1 / 2}\left(j+\frac{1}{2}\right)= & \frac{1-\sigma \Delta t / 2 \varepsilon_{0}}{1+\sigma \Delta t / 2 \varepsilon_{0}} H_{\mathrm{inc}}^{l-1 / 2}\left(j+\frac{1}{2}\right) \\
& -\frac{\Delta t}{\mu_{0} \Delta y} \frac{1}{1+\sigma \Delta t / 2 \varepsilon_{0}}\left[E_{\mathrm{inc}}^{l}(j+1)-E_{\mathrm{inc}}^{l}(j)\right] .
\end{aligned}
$$

4. Update the polarizations $\mathbf{P}$ and current $\mathbf{J}$

In the gain material region:

a. Pumped with a homogeneous pumping rate:

$$
\begin{aligned}
& P_{a x}^{l+1}\left(i+\frac{1}{2}, j, k\right)= \frac{2-\omega_{a}^{2} \Delta t^{2}}{1+\Gamma_{a} \Delta t / 2} P_{a x}^{l}\left(i+\frac{1}{2}, j, k\right)-\frac{1-\Gamma_{a} \Delta t / 2}{1+\Gamma_{a} \Delta t / 2} P_{a x}^{l-1}\left(i+\frac{1}{2}, j, k\right) \\
&+\frac{\sigma_{a} \Delta t^{2}}{1+\Gamma_{a} \Delta t / 2}\left[N_{1 x}^{l}\left(i+\frac{1}{2}, j, k\right)-N_{2 x}^{l}\left(i+\frac{1}{2}, j, k\right)\right] \\
& \cdot E_{x}^{l}\left(i+\frac{1}{2}, j, k\right), \\
& J_{p x}^{G} l+\frac{1}{2}\left(i+\frac{1}{2}, j, k\right)=\frac{1}{\Delta t}\left[P_{a x}^{l+1}\left(i+\frac{1}{2}, j, k\right)-P_{a x}^{l}\left(i+\frac{1}{2}, j, k\right)\right] \\
& P_{a y}^{l+1}\left(i, j+\frac{1}{2}, k\right)= \frac{2-\omega_{a}^{2} \Delta t^{2}}{1+\Gamma_{a} \Delta t / 2} P_{a y}^{l}\left(i, j+\frac{1}{2}, k\right)-\frac{1-\Gamma_{a} \Delta t / 2}{1+\Gamma_{a} \Delta t / 2} P_{a y}^{l-1}\left(i, j+\frac{1}{2}, k\right) \\
&+\frac{\sigma_{a} \Delta t^{2}}{1+\Gamma_{a} \Delta t / 2}\left[N_{1 y}^{l}\left(i, j+\frac{1}{2}, k\right)-N_{2 y}^{l}\left(i, j+\frac{1}{2}, k\right)\right]
\end{aligned}
$$




$$
\begin{gathered}
\cdot E_{y}^{l}\left(i, j+\frac{1}{2}, k\right), \\
J_{p y}^{G l+\frac{1}{2}}\left(i, j+\frac{1}{2}, k\right)=\frac{1}{\Delta t}\left[P_{a y}^{l+1}\left(i, j+\frac{1}{2}, k\right)-P_{a y}^{l}\left(i, j+\frac{1}{2}, k\right)\right], \\
P_{a z}^{l+1}\left(i, j, k+\frac{1}{2}\right)=\frac{2-\omega_{a}^{2} \Delta t^{2}}{1+\Gamma_{a} \Delta t / 2} P_{a z}^{l}\left(i, j, k+\frac{1}{2}\right)-\frac{1-\Gamma_{a} \Delta t / 2}{1+\Gamma_{a} \Delta t / 2} P_{a z}^{l-1}\left(i, j, k+\frac{1}{2}\right) \\
+\frac{\sigma_{a} \Delta t^{2}}{1+\Gamma_{a} \Delta t / 2}\left[N_{1 z}^{l}\left(i, j, k+\frac{1}{2}\right)-N_{2 z}^{l}\left(i, j, k+\frac{1}{2}\right)\right] \\
\cdot E_{z}^{l}\left(i, j, k+\frac{1}{2}\right), \\
J_{p z}^{G l+\frac{1}{2}}\left(i, j, k+\frac{1}{2}\right)=\frac{1}{\Delta t}\left[P_{a z}^{l+1}\left(i, j, k+\frac{1}{2}\right)-P_{a z}^{l}\left(i, j, k+\frac{1}{2}\right)\right] .
\end{gathered}
$$

\section{b. Optical pumping:}

In this case, the updates of $\mathbf{P}_{a}$ keep the same as the pumping rate case, but we need to do additional updates for $\mathbf{P}_{b}$, and the updates for the polarization current is changed.

$$
\begin{aligned}
P_{b x}^{l+1}\left(i+\frac{1}{2}, j, k\right)= & \frac{2-\omega_{b}^{2} \Delta t^{2}}{1+\Gamma_{b} \Delta t / 2} P_{b x}^{l}\left(i+\frac{1}{2}, j, k\right)-\frac{1-\Gamma_{b} \Delta t / 2}{1+\Gamma_{b} \Delta t / 2} P_{b x}^{l-1}\left(i+\frac{1}{2}, j, k\right) \\
& +\frac{\sigma_{b} \Delta t^{2}}{1+\Gamma_{b} \Delta t / 2}\left[N_{0 x}^{l}\left(i+\frac{1}{2}, j, k\right)-N_{3 x}^{l}\left(i+\frac{1}{2}, j, k\right)\right] \\
& \cdot E_{x}^{l}\left(i+\frac{1}{2}, j, k\right), \\
J_{p x}^{G} l+\frac{1}{2}\left(i+\frac{1}{2}, j, k\right)=\frac{1}{\Delta t}\left[P_{a x}^{l+1}\left(i+\frac{1}{2}, j, k\right)+P_{b x}^{l+1}\left(i+\frac{1}{2}, j, k\right)\right. & \left.-P_{a x}^{l}\left(i+\frac{1}{2}, j, k\right)-P_{b x}^{l}\left(i+\frac{1}{2}, j, k\right)\right], \\
P_{b y}^{l+1}\left(i, j+\frac{1}{2}, k\right)= & \frac{2-\omega_{b}^{2} \Delta t^{2}}{1+\Gamma_{b} \Delta t / 2} P_{b y}^{l}\left(i, j+\frac{1}{2}, k\right)-\frac{1-\Gamma_{b} \Delta t / 2}{1+\Gamma_{b} \Delta t / 2} P_{b y}^{l-1}\left(i, j+\frac{1}{2}, k\right) \\
& +\frac{\sigma_{b} \Delta t^{2}}{1+\Gamma_{b} \Delta t / 2}\left[N_{0 y}^{l}\left(i, j+\frac{1}{2}, k\right)-N_{3 y}^{l}\left(i, j+\frac{1}{2}, k\right)\right]
\end{aligned}
$$




$$
\begin{gathered}
\cdot E_{y}^{l}\left(i, j+\frac{1}{2}, k\right), \\
J_{p y}^{G l+\frac{1}{2}}\left(i, j+\frac{1}{2}, k\right)=\frac{1}{\Delta t}\left[P_{a y}^{l+1}\left(i, j+\frac{1}{2}, k\right)+P_{b y}^{l+1}\left(i, j+\frac{1}{2}, k\right)\right. \\
\left.-P_{a y}^{l}\left(i, j+\frac{1}{2}, k\right)-P_{b y}^{l}\left(i, j+\frac{1}{2}, k\right)\right], \\
P_{b z}^{l+1}\left(i, j, k+\frac{1}{2}\right)=\frac{2-\omega_{b}^{2} \Delta t^{2}}{1+\Gamma_{b} \Delta t / 2} P_{b z}^{l}\left(i, j, k+\frac{1}{2}\right)-\frac{1-\Gamma_{b} \Delta t / 2}{1+\Gamma_{b} \Delta t / 2} P_{b z}^{l-1}\left(i, j, k+\frac{1}{2}\right) \\
+\frac{\sigma_{b} \Delta t^{2}}{1+\Gamma_{b} \Delta t / 2}\left[N_{0 z}^{l}\left(i, j, k+\frac{1}{2}\right)-N_{3 z}^{l}\left(i, j, k+\frac{1}{2}\right)\right] \\
\cdot E_{z}^{l}\left(i, j, k+\frac{1}{2}\right), \\
\left.\quad-P_{a z}^{l}\left(i, j, k+\frac{1}{2}\right)-P_{b z}^{l}\left(i, j, k+\frac{1}{2}\right)\right] . \\
J_{p z}^{G l+\frac{1}{2}}\left(i, j, k+\frac{1}{2}\right)=\frac{1}{\Delta t}\left[P_{a z}^{l+1}\left(i, j, k+\frac{1}{2}\right)+P_{b z}^{l+1}\left(i, j, k+\frac{1}{2}\right)\right.
\end{gathered}
$$

In the Drude metal region,

$$
\begin{aligned}
J_{p x}^{l+\frac{1}{2}}\left(i+\frac{1}{2}, j, k\right)= & \frac{1-0.5 \Gamma_{e x} \Delta t}{1+0.5 \Gamma_{e x} \Delta t} J_{p x}^{l-\frac{1}{2}}\left(i+\frac{1}{2}, j, k\right) \\
& +\frac{\varepsilon_{0} \omega_{e p x}^{2} \Delta t}{1+0.5 \Gamma_{e x} \Delta t} E_{x}^{l}\left(i+\frac{1}{2}, j, k\right), \\
J_{p y}^{l+\frac{1}{2}}\left(i, j+\frac{1}{2}, k\right)= & \frac{1-0.5 \Gamma_{e y} \Delta t}{1+0.5 \Gamma_{e y} \Delta t} J_{p y}^{l-\frac{1}{2}}\left(i, j+\frac{1}{2}, k\right) \\
& +\frac{\varepsilon_{0} \omega_{e p y}^{2} \Delta t}{1+0.5 \Gamma_{e y} \Delta t} E_{y}^{l}\left(i, j+\frac{1}{2}, k\right), \\
J_{p z}^{l+\frac{1}{2}}\left(i, j, k+\frac{1}{2}\right)= & \frac{1-0.5 \Gamma_{e z} \Delta t}{1+0.5 \Gamma_{e z} \Delta t} J_{p z}^{l-\frac{1}{2}}\left(i, j, k+\frac{1}{2}\right) \\
& +\frac{\varepsilon_{0} \omega_{e p z}^{2} \Delta t}{1+0.5 \Gamma_{e z} \Delta t} E_{z}^{l}\left(i, j, k+\frac{1}{2}\right) .
\end{aligned}
$$


5. Update the electric field components $E_{x}, E_{y}$ and $E_{z}$

Here $\mathbf{J}$ is the polarization current $\mathbf{J}_{p}$ in gain materials and Drude metals and the source current in lossy dielectrics.

In the lossy dielectric, gain material and Drude metal regions,

$$
\begin{aligned}
E_{x}^{l+1}\left(i+\frac{1}{2}, j, k\right)= & \frac{1-\sigma_{x} \Delta t / 2 \varepsilon_{x}}{1+\sigma_{x} \Delta t / 2 \varepsilon_{x}} E_{x}^{l}\left(i+\frac{1}{2}, j, k\right) \\
& +\frac{\Delta t / \varepsilon_{x}}{1+\sigma_{x} \Delta t / 2 \varepsilon_{x}}\left(\frac{H_{z}^{l+1 / 2}\left(i+\frac{1}{2}, j+\frac{1}{2}, k\right)-H_{z}^{l+1 / 2}\left(i+\frac{1}{2}, j-\frac{1}{2}, k\right)}{\Delta y}\right. \\
& -\frac{H_{y}^{l+1 / 2}\left(i+\frac{1}{2}, j, k+\frac{1}{2}\right)-H_{y}^{l+1 / 2}\left(i+\frac{1}{2}, j, k-\frac{1}{2}\right)}{\Delta z} \\
& \left.-J_{x}^{l+\frac{1}{2}}\left(i+\frac{1}{2}, j, k\right)\right), \\
E_{y}^{l+1}\left(i, j+\frac{1}{2}, k\right)= & \frac{1-\sigma_{y} \Delta t / 2 \varepsilon_{y}}{1+\sigma_{y} \Delta t / 2 \varepsilon_{y}} E_{y}^{l}\left(i, j+\frac{1}{2}, k\right) \\
& +\frac{\Delta t / \varepsilon_{y}}{1+\sigma_{y} \Delta t / 2 \varepsilon_{y}}\left(\frac{H_{x}^{l+1 / 2}\left(i, j+\frac{1}{2}, k+\frac{1}{2}\right)-H_{x}^{l+1 / 2}\left(i, j+\frac{1}{2}, k-\frac{1}{2}\right)}{\Delta z}\right. \\
& -\frac{H_{z}^{l+1 / 2}\left(i+\frac{1}{2}, j+\frac{1}{2}, k\right)-H_{z}^{l+1 / 2}\left(i-\frac{1}{2}, j+\frac{1}{2}, k\right)}{\Delta x} \\
& \left.-J_{y}^{l+\frac{1}{2}}\left(i, j+\frac{1}{2}, k\right)\right), \\
& -\frac{\left.H_{z}^{l+\frac{1}{2}}\left(i, j, k+\frac{1}{2}\right)\right) \cdot}{\Delta+\sigma_{z} \Delta t / 2 \varepsilon_{z}}\left(\frac{H_{y}^{l+1 / 2}\left(i, j+\frac{1}{2}, k+\frac{1}{2}\right)-H_{x}^{l+1 / 2}\left(i, j-\frac{1}{2}, k+\frac{1}{2}\right)}{\Delta y}\right. \\
E_{z}^{l+1}\left(i, j, k+\frac{\mathrm{A}}{2}\right)= & \frac{1-\sigma_{z} \Delta t / 2 \varepsilon_{z}}{1+\sigma_{z} \Delta t / 2 \varepsilon_{z}} E_{z}^{l}\left(i, j, k+\frac{1}{2}\right) \\
& \frac{\Delta t / \varepsilon_{z}}{1+1 / 2}\left(i+\frac{1}{2}, j, k+\frac{1}{2}\right)-H_{y}^{l+1 / 2}\left(i-\frac{1}{2}, j, k+\frac{1}{2}\right) \\
& \\
& \\
& \\
&
\end{aligned}
$$

In the PML region,

$$
E_{x y}^{l+1}\left(i+\frac{1}{2}, j, k\right)=\frac{1-\sigma_{y} \Delta t / 2 \varepsilon}{1+\sigma_{y} \Delta t / 2 \varepsilon} E_{x y}^{l}\left(i+\frac{1}{2}, j, k\right)+\frac{\Delta t / \Delta y \varepsilon}{1+\sigma_{y} \Delta t / 2 \varepsilon}
$$




$$
\begin{aligned}
& \cdot\left[H_{z}^{l+1 / 2}\left(i+\frac{1}{2}, j+\frac{1}{2}, k\right)-H_{z}^{l+1 / 2}\left(i+\frac{1}{2}, j-\frac{1}{2}, k\right)\right], \\
& E_{x z}^{l+1}\left(i+\frac{1}{2}, j, k\right)=\frac{1-\sigma_{z} \Delta t / 2 \varepsilon}{1+\sigma_{z} \Delta t / 2 \varepsilon} E_{x z}^{l}\left(i+\frac{1}{2}, j, k\right)-\frac{\Delta t / \Delta z \varepsilon}{1+\sigma_{z} \Delta t / 2 \varepsilon} \\
& \cdot\left[H_{y}^{l+1 / 2}\left(i+\frac{1}{2}, j, k+\frac{1}{2}\right)-H_{y}^{l+1 / 2}\left(i+\frac{1}{2}, j, k-\frac{1}{2}\right)\right] \text {, } \\
& E_{x}^{l+1}\left(i+\frac{1}{2}, j, k\right)=E_{x y}^{l+1}\left(i+\frac{1}{2}, j, k\right)+E_{x z}^{l+1}\left(i+\frac{1}{2}, j, k\right) . \\
& E_{y z}^{l+1}\left(i, j+\frac{1}{2}, k\right)=\frac{1-\sigma_{z} \Delta t / 2 \varepsilon}{1+\sigma_{z} \Delta t / 2 \varepsilon} E_{y z}^{l}\left(i, j+\frac{1}{2}, k\right)+\frac{\Delta t / \Delta z \varepsilon}{1+\sigma_{z} \Delta t / 2 \varepsilon} \\
& \cdot\left[H_{x}^{l+1 / 2}\left(i, j+\frac{1}{2}, k+\frac{1}{2}\right)-H_{x}^{l+1 / 2}\left(i, j+\frac{1}{2}, k-\frac{1}{2}\right)\right] \text {, } \\
& E_{y x}^{l+1}\left(i, j+\frac{1}{2}, k\right)=\frac{1-\sigma_{x} \Delta t / 2 \varepsilon}{1+\sigma_{x} \Delta t / 2 \varepsilon} E_{y x}^{l}\left(i, j+\frac{1}{2}, k\right)-\frac{\Delta t / \Delta x \varepsilon}{1+\sigma_{x} \Delta t / 2 \varepsilon} \\
& \cdot\left[H_{z}^{l+1 / 2}\left(i+\frac{1}{2}, j+\frac{1}{2}, k\right)-H_{z}^{l+1 / 2}\left(i-\frac{1}{2}, j+\frac{1}{2}, k\right)\right] \text {, } \\
& E_{y}^{l+1}\left(i, j+\frac{1}{2}, k\right)=E_{y z}^{l+1}\left(i, j+\frac{1}{2}, k\right)+E_{y x}^{l+1}\left(i, j+\frac{1}{2}, k\right) . \\
& E_{z x}^{l+1}\left(i, j, k+\frac{1}{2}\right)=\frac{1-\sigma_{x} \Delta t / 2 \varepsilon}{1+\sigma_{x} \Delta t / 2 \varepsilon} E_{z x}^{l}\left(i, j, k+\frac{1}{2}\right)+\frac{\Delta t / \Delta x \varepsilon}{1+\sigma_{x} \Delta t / 2 \varepsilon} \\
& \cdot\left[H_{y}^{l+1 / 2}\left(i+\frac{1}{2}, j, k+\frac{1}{2}\right)-H_{y}^{l+1 / 2}\left(i-\frac{1}{2}, j, k+\frac{1}{2}\right)\right] \text {, } \\
& E_{z y}^{l+1}\left(i, j, k+\frac{1}{2}\right)=\frac{1-\sigma_{y} \Delta t / 2 \varepsilon}{1+\sigma_{y} \Delta t / 2 \varepsilon} E_{z y}^{l}\left(i, j, k+\frac{1}{2}\right)-\frac{\Delta t / \Delta y \varepsilon}{1+\sigma_{y} \Delta t / 2 \varepsilon} \\
& \cdot\left[H_{x}^{l+1 / 2}\left(i, j+\frac{1}{2}, k+\frac{1}{2}\right)-H_{x}^{l+1 / 2}\left(i, j-\frac{1}{2}, k+\frac{1}{2}\right)\right] \text {, } \\
& E_{z}^{l+1}\left(i, j, k+\frac{1}{2}\right)=E_{z x}^{l+1}\left(i, j, k+\frac{1}{2}\right)+E_{z y}^{l+1}\left(i, j, k+\frac{1}{2}\right) .
\end{aligned}
$$

6. Make $E_{x}$ and $E_{z}$ corrections over the connecting interfaces (TF/SF method) 
The incident field components needed to implement the correction formula for $E_{x}$ and $E_{z}$,

$$
\begin{aligned}
& H_{z, \text { inc }}^{l+1 / 2}\left(i+\frac{1}{2}, j_{L}-\frac{1}{2}, k\right)=H_{\mathrm{inc}}^{l+1 / 2}\left(j_{L}-\frac{1}{2}\right) \sin \theta \\
& H_{z, \text { inc }}^{l+1 / 2}\left(i+\frac{1}{2}, j_{R}+\frac{1}{2}, k\right)=H_{\mathrm{inc}}^{l+1 / 2}\left(j_{R}+\frac{1}{2}\right) \sin \theta \\
& H_{x, \text { inc }}^{l+1 / 2}\left(i, j_{L}-\frac{1}{2}, k+\frac{1}{2}\right)=H_{\mathrm{inc}}^{l+1 / 2}\left(j_{L}-\frac{1}{2}\right) \cos \theta \\
& H_{x, \text { inc }}^{l+1 / 2}\left(i, j_{R}+\frac{1}{2}, k+\frac{1}{2}\right)=H_{\mathrm{inc}}^{l+1 / 2}\left(j_{R}+\frac{1}{2}\right) \cos \theta
\end{aligned}
$$

Implement the correction formula for $E_{x}$ and $E_{z}$ as follows,

6a. Make $E_{x}$ corrections

At the left interface $j=j_{L}$,

$$
E_{x}^{l+1}\left(i+\frac{1}{2}, j_{L}, k\right)=\left\{E_{x}^{l+1}\left(i+\frac{1}{2}, j_{L}, k\right)\right\}-\frac{\Delta t}{\Delta y \varepsilon_{0}} H_{z, \text { inc }}^{l+1 / 2}\left(i+\frac{1}{2}, j_{L}-\frac{1}{2}, k\right)
$$

At the right interface $j=j_{R}$,

$$
E_{x}^{l+1}\left(i+\frac{1}{2}, j_{R}, k\right)=\left\{E_{x}^{l+1}\left(i+\frac{1}{2}, j_{R}, k\right)\right\}+\frac{\Delta t}{\Delta y \varepsilon_{0}} H_{z, \text { inc }}^{l+1 / 2}\left(i+\frac{1}{2}, j_{R}+\frac{1}{2}, k\right) .
$$

6b. Make $E_{z}$ corrections

At the left interface $j=j_{L}$,

$$
E_{z}^{l+1}\left(i, j_{L}, k+\frac{1}{2}\right)=\left\{E_{z}^{l+1}\left(i, j_{L}, k+\frac{1}{2}\right)\right\}+\frac{\Delta t}{\Delta y \varepsilon_{0}} H_{x, \text { inc }}^{l+1 / 2}\left(i, j_{L}-\frac{1}{2}, k+\frac{1}{2}\right) .
$$

At the right interface $j=j_{R}$,

$$
E_{z}^{l+1}\left(i, j_{R}, k+\frac{1}{2}\right)=\left\{E_{z}^{l+1}\left(i, j_{R}, k+\frac{1}{2}\right)\right\}-\frac{\Delta t}{\Delta y \varepsilon_{0}} H_{x, \text { inc }}^{l+1 / 2}\left(i, j_{R}+\frac{1}{2}, k+\frac{1}{2}\right) .
$$

7. Update $E_{\text {inc }}$ in the source grid generating look-up table

$$
E_{\text {inc }}^{l+1}(j)=\frac{1-\sigma \Delta t / 2 \varepsilon_{0}}{1+\sigma \Delta t / 2 \varepsilon_{0}} E_{\text {inc }}^{l}(j)
$$




$$
-\frac{\Delta t}{\varepsilon_{0} \Delta y} \frac{1}{1+\sigma \Delta t / 2 \varepsilon_{0}}\left[H_{\mathrm{inc}}^{l+1 / 2}\left(j+\frac{1}{2}\right)-H_{\mathrm{inc}}^{l+1 / 2}\left(j-\frac{1}{2}\right)\right] .
$$

8. Update the occupation numbers $N_{0}, N_{1}, N_{2}$, and $N_{3}$

8a. Update the occupation number $N_{3}$

(1). Pumped with a homogeneous pumping rate:

$$
\begin{aligned}
N_{3 x}^{l+1}\left(i+\frac{1}{2}, j, k\right)= & \frac{1-\Delta t / 2 \tau_{32}}{1+\Delta t / 2 \tau_{32}} N_{3 x}^{l}\left(i+\frac{1}{2}, j, k\right) \\
& +\frac{\Delta t \Gamma_{\text {pump }, x}}{1+\Delta t / 2 \tau_{32}} N_{0 x}^{l}\left(i+\frac{1}{2}, j, k\right), \\
N_{3 y}^{l+1}\left(i, j+\frac{1}{2}, k\right)= & \frac{1-\Delta t / 2 \tau_{32}}{1+\Delta t / 2 \tau_{32}} N_{3 y}^{l}\left(i, j+\frac{1}{2}, k\right) \\
& +\frac{\Delta t \Gamma_{\text {pump }, y}}{1+\Delta t / 2 \tau_{32}} N_{0 y}^{l}\left(i, j+\frac{1}{2}, k\right), \\
N_{3 z}^{l+1}\left(i, j, k+\frac{1}{2}\right)= & \frac{1-\Delta t / 2 \tau_{32}}{1+\Delta t / 2 \tau_{32}} N_{3 z}^{l}\left(i, j, k+\frac{1}{2}\right) \\
& +\frac{\Delta t \Gamma_{\text {pump }, z}}{1+\Delta t / 2 \tau_{32}} N_{0 z}^{l}\left(i, j, k+\frac{1}{2}\right) .
\end{aligned}
$$

(2). Optical pumping:

$$
\begin{aligned}
N_{3 x}^{l+1}\left(i+\frac{1}{2}, j, k\right)= & \frac{1-\Delta t / 2 \tau_{32}}{1+\Delta t / 2 \tau_{32}} N_{3 x}^{l}\left(i+\frac{1}{2}, j, k\right) \\
& +\frac{1}{2 \hbar \omega_{b}} \frac{1}{1+\Delta t / 2 \tau_{32}}\left[E_{x}^{l+1}\left(i+\frac{1}{2}, j, k\right)+E_{x}^{l}\left(i+\frac{1}{2}, j, k\right)\right] \\
& \cdot\left[P_{b x}^{l+1}\left(i+\frac{1}{2}, j, k\right)-P_{b x}^{l}\left(i+\frac{1}{2}, j, k\right)\right], \\
N_{3 y}^{l+1}\left(i, j+\frac{1}{2}, k\right)= & \frac{1-\Delta t / 2 \tau_{32}}{1+\Delta t / 2 \tau_{32}} N_{3 y}^{l}\left(i, j+\frac{1}{2}, k\right) \\
& +\frac{1}{2 \hbar \omega_{b}} \frac{1}{1+\Delta t / 2 \tau_{32}}\left[E_{y}^{l+1}\left(i, j+\frac{1}{2}, k\right)+E_{y}^{l}\left(i, j+\frac{1}{2}, k\right)\right] \\
& \cdot\left[P_{b y}^{l+1}\left(i, j+\frac{1}{2}, k\right)-P_{b y}^{l}\left(i, j+\frac{1}{2}, k\right)\right],
\end{aligned}
$$




$$
\begin{aligned}
N_{3 z}^{l+1}\left(i, j, k+\frac{1}{2}\right)= & \frac{1-\Delta t / 2 \tau_{32}}{1+\Delta t / 2 \tau_{32}} N_{3 z}^{l}\left(i, j, k+\frac{1}{2}\right) \\
& +\frac{1}{2 \hbar \omega_{b}} \frac{1}{1+\Delta t / 2 \tau_{32}}\left[E_{z}^{l+1}\left(i, j, k+\frac{1}{2}\right)+E_{z}^{l}\left(i, j, k+\frac{1}{2}\right)\right] \\
& \cdot\left[P_{b z}^{l+1}\left(i, j, k+\frac{1}{2}\right)-P_{b z}^{l}\left(i, j, k+\frac{1}{2}\right)\right] .
\end{aligned}
$$

8b. Update the occupation number $N_{2}$

$$
\begin{aligned}
& N_{2 x}^{l+1}\left(i+\frac{1}{2}, j, k\right)=\frac{1-\Delta t / 2 \tau_{21}}{1+\Delta t / 2 \tau_{21}} N_{2 x}^{l}\left(i+\frac{1}{2}, j, k\right) \\
& +\frac{1}{2 \hbar \omega_{a}} \frac{1}{1+\Delta t / 2 \tau_{21}}\left[E_{x}^{l+1}\left(i+\frac{1}{2}, j, k\right)+E_{x}^{l}\left(i+\frac{1}{2}, j, k\right)\right] \\
& \cdot\left[P_{a x}^{l+1}\left(i+\frac{1}{2}, j, k\right)-P_{a x}^{l}\left(i+\frac{1}{2}, j, k\right)\right] \\
& +\frac{1}{1+\Delta t / 2 \tau_{21}} \frac{\Delta t}{2 \tau_{32}}\left[N_{3 x}^{l+1}\left(i+\frac{1}{2}, j, k\right)+N_{3 x}^{l}\left(i+\frac{1}{2}, j, k\right)\right], \\
& N_{2 y}^{l+1}\left(i, j+\frac{1}{2}, k\right)=\frac{1-\Delta t / 2 \tau_{21}}{1+\Delta t / 2 \tau_{21}} N_{2 y}^{l}\left(i, j+\frac{1}{2}, k\right) \\
& +\frac{1}{2 \hbar \omega_{a}} \frac{1}{1+\Delta t / 2 \tau_{21}}\left[E_{y}^{l+1}\left(i, j+\frac{1}{2}, k\right)+E_{y}^{l}\left(i, j+\frac{1}{2}, k\right)\right] \\
& \cdot\left[P_{a y}^{l+1}\left(i, j+\frac{1}{2}, k\right)-P_{a y}^{l}\left(i, j+\frac{1}{2}, k\right)\right] \\
& +\frac{1}{1+\Delta t / 2 \tau_{21}} \frac{\Delta t}{2 \tau_{32}}\left[N_{3 y}^{l+1}\left(i, j+\frac{1}{2}, k\right)+N_{3 y}^{l}\left(i, j+\frac{1}{2}, k\right)\right], \\
& N_{2 z}^{l+1}\left(i, j, k+\frac{1}{2}\right)=\frac{1-\Delta t / 2 \tau_{21}}{1+\Delta t / 2 \tau_{21}} N_{2 z}^{l}\left(i, j, k+\frac{1}{2}\right) \\
& +\frac{1}{2 \hbar \omega_{a}} \frac{1}{1+\Delta t / 2 \tau_{21}}\left[E_{z}^{l+1}\left(i, j, k+\frac{1}{2}\right)+E_{z}^{l}\left(i, j, k+\frac{1}{2}\right)\right] \\
& \cdot\left[P_{a z}^{l+1}\left(i, j, k+\frac{1}{2}\right)-P_{a z}^{l}\left(i, j, k+\frac{1}{2}\right)\right] \\
& +\frac{1}{1+\Delta t / 2 \tau_{21}} \frac{\Delta t}{2 \tau_{32}}\left[N_{3 z}^{l+1}\left(i, j, k+\frac{1}{2}\right)+N_{3 z}^{l}\left(i, j, k+\frac{1}{2}\right)\right] .
\end{aligned}
$$

8c. Update the occupation number $N_{1}$

$$
N_{1 x}^{l+1}\left(i+\frac{1}{2}, j, k\right)=\frac{1-\Delta t / 2 \tau_{10}}{1+\Delta t / 2 \tau_{10}} N_{1 x}^{l}\left(i+\frac{1}{2}, j, k\right)
$$




$$
\begin{aligned}
& -\frac{1}{2 \hbar \omega_{a}} \frac{1}{1+\Delta t / 2 \tau_{10}}\left[E_{x}^{l+1}\left(i+\frac{1}{2}, j, k\right)+E_{x}^{l}\left(i+\frac{1}{2}, j, k\right)\right] \\
& \cdot\left[P_{a x}^{l+1}\left(i+\frac{1}{2}, j, k\right)-P_{a x}^{l}\left(i+\frac{1}{2}, j, k\right)\right] \\
& +\frac{1}{1+\Delta t / 2 \tau_{10}} \frac{\Delta t}{2 \tau_{21}}\left[N_{2 x}^{l+1}\left(i+\frac{1}{2}, j, k\right)+N_{2 x}^{l}\left(i+\frac{1}{2}, j, k\right)\right],
\end{aligned}
$$

$$
\begin{aligned}
N_{1 y}^{l+1}\left(i, j+\frac{1}{2}, k\right)= & \frac{1-\Delta t / 2 \tau_{10}}{1+\Delta t / 2 \tau_{10}} N_{1 y}^{l}\left(i, j+\frac{1}{2}, k\right) \\
& -\frac{1}{2 \hbar \omega_{a}} \frac{1}{1+\Delta t / 2 \tau_{10}}\left[E_{y}^{l+1}\left(i, j+\frac{1}{2}, k\right)+E_{y}^{l}\left(i, j+\frac{1}{2}, k\right)\right] \\
& \cdot\left[P_{a y}^{l+1}\left(i, j+\frac{1}{2}, k\right)-P_{a y}^{l}\left(i, j+\frac{1}{2}, k\right)\right] \\
& +\frac{1}{1+\Delta t / 2 \tau_{10}} \frac{\Delta t}{2 \tau_{21}}\left[N_{2 y}^{l+1}\left(i, j+\frac{1}{2}, k\right)+N_{2 y}^{l}\left(i, j+\frac{1}{2}, k\right)\right],
\end{aligned}
$$

$$
\begin{aligned}
N_{1 z}^{l+1}\left(i, j, k+\frac{1}{2}\right)= & \frac{1-\Delta t / 2 \tau_{10}}{1+\Delta t / 2 \tau_{10}} N_{1 z}^{l}\left(i, j, k+\frac{1}{2}\right) \\
& -\frac{1}{2 \hbar \omega_{a}} \frac{1}{1+\Delta t / 2 \tau_{10}}\left[E_{z}^{l+1}\left(i, j, k+\frac{1}{2}\right)+E_{z}^{l}\left(i, j, k+\frac{1}{2}\right)\right] \\
& \cdot\left[P_{a z}^{l+1}\left(i, j, k+\frac{1}{2}\right)-P_{a z}^{l}\left(i, j, k+\frac{1}{2}\right)\right] \\
& +\frac{1}{1+\Delta t / 2 \tau_{10}} \frac{\Delta t}{2 \tau_{21}}\left[N_{2 z}^{l+1}\left(i, j, k+\frac{1}{2}\right)+N_{2 z}^{l}\left(i, j, k+\frac{1}{2}\right)\right] .
\end{aligned}
$$

8d. Update the occupation number $N_{0}$

(1). Pumped with a homogeneous pumping rate:

$$
\begin{aligned}
N_{0 x}^{l+1}\left(i+\frac{1}{2}, j, k\right)= & \left(1-\Gamma_{\text {pump }, x} \Delta t\right) N_{0 x}^{l}\left(i+\frac{1}{2}, j, k\right) \\
& +\frac{\Delta t}{2 \tau_{10}}\left[N_{1 x}^{l+1}\left(i+\frac{1}{2}, j, k\right)+N_{1 x}^{l}\left(i+\frac{1}{2}, j, k\right)\right], \\
N_{0 y}^{l+1}\left(i, j+\frac{1}{2}, k\right)= & \left(1-\Gamma_{\text {pump }, y} \Delta t\right) N_{0 y}^{l}\left(i, j+\frac{1}{2}, k\right) \\
& +\frac{\Delta t}{2 \tau_{10}}\left[N_{1 y}^{l+1}\left(i, j+\frac{1}{2}, k\right)+N_{1 y}^{l}\left(i, j+\frac{1}{2}, k\right)\right],
\end{aligned}
$$




$$
\begin{aligned}
N_{0 z}^{l+1}\left(i, j, k+\frac{1}{2}\right)= & \left(1-\Gamma_{\text {pump }, z} \Delta t\right) N_{0 z}^{l}\left(i, j, k+\frac{1}{2}\right) \\
& +\frac{\Delta t}{2 \tau_{10}}\left[N_{1 z}^{l+1}\left(i, j, k+\frac{1}{2}\right)+N_{1 z}^{l}\left(i, j, k+\frac{1}{2}\right)\right] .
\end{aligned}
$$

(2). Optical pumping:

$$
\begin{aligned}
N_{0 x}^{l+1}\left(i+\frac{1}{2}, j, k\right)= & N_{0 x}^{l}\left(i+\frac{1}{2}, j, k\right)-\frac{1}{2 \hbar \omega_{b}}\left[E_{x}^{l+1}\left(i+\frac{1}{2}, j, k\right)+E_{x}^{l}\left(i+\frac{1}{2}, j, k\right)\right] \\
& \cdot\left[P_{b x}^{l+1}\left(i+\frac{1}{2}, j, k\right)-P_{b x}^{l}\left(i+\frac{1}{2}, j, k\right)\right] \\
& +\frac{\Delta t}{2 \tau_{10}}\left[N_{1 x}^{l+1}\left(i+\frac{1}{2}, j, k\right)+N_{1 x}^{l}\left(i+\frac{1}{2}, j, k\right)\right], \\
N_{0 y}^{l+1}\left(i, j+\frac{1}{2}, k\right)= & N_{0 y}^{l}\left(i, j+\frac{1}{2}, k\right)-\frac{1}{2 \hbar \omega_{b}}\left[E_{y}^{l+1}\left(i, j+\frac{1}{2}, k\right)+E_{y}^{l}\left(i, j+\frac{1}{2}, k\right)\right] \\
& \cdot\left[P_{b y}^{l+1}\left(i, j+\frac{1}{2}, k\right)-P_{b y}^{l}\left(i, j+\frac{1}{2}, k\right)\right] \\
& +\frac{\Delta t}{2 \tau_{10}}\left[N_{1 y}^{l+1}\left(i, j+\frac{1}{2}, k\right)+N_{1 y}^{l}\left(i, j+\frac{1}{2}, k\right)\right], \\
& \cdot\left[P_{b z}^{l+1}\left(i, j, k+\frac{1}{2}\right)-P_{b z}^{l}\left(i, j, k+\frac{1}{2}\right)\right] \\
& +\frac{\Delta t}{2 \tau_{10}}\left[N_{1 z}^{l+1}\left(i, j, k+\frac{1}{2}\right)+N_{1 z}^{l}\left(i, j, k+\frac{1}{2}\right)\right] . \\
N_{0 z}^{l+1}\left(i, j, k+\frac{1}{2}\right)= & N_{0 z}^{l}\left(i, j, k+\frac{1}{2}\right)-\frac{1}{2 \hbar \omega_{b}}\left[E_{z}^{l+1}\left(i, j, k+\frac{1}{2}\right)+E_{z}^{l}\left(i, j, k+\frac{1}{2}\right)\right] \\
&
\end{aligned}
$$




\section{BIBLIOGRAPHY}

[1] V. G. Veselago, Sov. Phys. Usp. 10, 509 (1968).

[2] J. B. Pendry, Phys. Rev. Lett. 85, 3966 (2000).

[3] J. B. Pendry, D. Schurig, and D. R. Smith, Science 312, 1780 (2006).

[4] D. R. Smith, J. B. Pendry, and M. C. K. Wiltshire, Science 305, 788 (2004).

[5] C. M. Soukoulis, M. Kafesaki, and E. N. Economou, Adv. Mater. 18, 1941 (2006).

[6] V. M. Shalaev, Nature Photon. 1, 41 (2007).

[7] C. M. Soukoulis, S. Linden, and M. Wegener, Science 315, 47 (2007).

[8] J. B. Pendry, Contemp. Phys. 45, 191 (2004).

[9] S. A. Ramakrishna, Rep. Prog. Phys. 68, 449 (2005).

[10] F. Capolino, Theory and Phenomena of Metamaterials (Taylor \& Francis Group, Boca Raton, FL, 2009).

[11] K. Busch, G. V. Freymann, S. Linden, S. F. Mingaleev, L. Tkeshelashvili, and M. Wegener, Phys. Rep. 444, 101 (2007).

[12] N. I. Zheludev, Science 328, 582 (2010).

[13] J. B. Pendry, A. Holden, D. Robbins, W. Stewart, IEEE Trans. Microwave Theory Tech. 47, 2075 (1999). 
[14] D. R. Smith, W. J. Padilla, D. C. Vier, S. C. Nemat-Nasser, and S. Schultz, Phys. Rev. Lett. 84, 4184 (2000).

[15] J. B. Pendry, A. J. Holden, W. J. Stewart, I. Youngs, Phys. Rev. Lett. 76, 4773 (1996).

[16] R. Shelby, D. R. Smith, and S. Schultz, Science 292, 77 (2001).

[17] A. A. Houch, J. B. Brock, and I. L. Chuang, Phys. Rev. Lett. 90, 137401 (2003).

[18] C. G. Parazzoli, R. B. Greegor, K. Li, B. E. C. Koltenbah, and M. Tanielian, Phys. Rev. Lett. 90, 107401 (2003).

[19] T. J. Yen, W. J. Padilla, N. Fang, D. C. Vier, D. R. Smith, J. B. Pendry, D. N. Basov, and X. Zhang, Science 303, 1494 (2004).

[20] S. Linden, C. Enkrich, M. Wegener, J. Zhou, T. Koschny, and C. M. Soukoulis, Science 306, 1351 (2004).

[21] N. Katsarakis, G. Konstantinidis, A. Kostopoulos, R. S. Penciu, T. F. Gundogdu, Th. Koschny, M. Kafesaki, E. N. Economou, and C. M. Soukoulis, Opt. Lett. 30, 1348 (2005).

[22] C. Enkrich, S. Linden, M. Wegener, S. Burger, L. Zswchiedrich, F. Schmidt, J. Zhou, T. Koschny, and C. M. Soukoulis, Phys. Rev. Lett. 95, 203901 (2005).

[23] C. Enkrich, F. Perez-Willard, D. Gerthsen, J. Zhou, T. Koschny, C. M. Soukoulis, M. Wegener, and S. Linden, Adv. Mater. 17, 2543 (2005).

[24] G. Dolling, C. Enkrich, M. Wegener, J. F. Zhou, and C. M. Soukoulis, Opt. Lett. 30, 3198 $(2005)$

[25] H. K. Yuan, U. K. Chettiar, W. S. Cai, A. V. Kildishev, A. Boltasseva, V. P. Drachev, V. M. Shalaev, Opt. Express 15, 1076 (2007).

[26] S. Zhang, W. J. Fan, N. C. Panoiu, K. J. Malloy, R. M. Osgood, and S. R. J. Brueck, Phys. Rev. Lett. 95, 137404 (2005). 
[27] V. M. Shalaev, W. S. Cai, U. K. Chettiar, H. K. Yuan, A. K. Sarychev, V. P. Drachev, and A. V. Kildishev, Opt. Lett. 30, 3356 (2005).

[28] J. Valentine, S. Zhang, T. Zentgraf, E. Ulin-Avila, D. A. Genov, G. Bartal, and X. Zhang, Nature 455, 376 (2008).

[29] G. Dolling, C. Enkrich, M. Wegener, C. M. Soukoulis, and S. Linden, Opt. Lett. 31, 1800 (2006).

[30] G. Dolling, M. Wegener, C. M. Soukoulis, and S. Linden, Opt. Lett. 32, 53 (2007).

[31] Z. Liu, X. Zhang, Y. Mao, Y. Y. Zhu, Z. Yang, C. T. Chan, and P. Sheng, Science 289, 1734 (2000).

[32] S. Guenneau, A. Movchan, G. Petursson, and S. A. Ramakrishna, New J. Phys. 9, 399 (2007).

[33] M. Brun, S. Guenneau, and A. B. Movchan, Appl. Phys. Lett. 94, 061903 (2009).

[34] M. H. Lu, C. Zhang, L. Feng, J. Zhao, Y. F. Chen, Y. W. Mao, J. Zi, Y. Y. Zhu, S. N. Zhu, and N. B. Ming, Nature Mater. 6, 744 (2007).

[35] W. S. Cai, U. K. Chettiar, A. V. Kildishev, and V. M. Shalaev, Nature Photon. 1, 224 (2007).

[36] D. Schurig, J. J. Mock, B. J. Justine, S. A. Cummer, J. B. Pendry, A. F. Starr, and D. R. Smith, Science 314, 977 (2006).

[37] U. Leonhardt, Science 312, 1777 (2006).

[38] U. Leonhardt, New J. Phys. 8, 118 (2006).

[39] A. A. Zharov, I. V. Shadrivov, and Y. S. Kivshar, Phys. Rev. Lett. 91, 037401 (2003).

[40] S. O’Brien, D. McPeake, S. A. Ramakrishna, and J. B. Pendry, Phys. Rev. B 69, 241101 (2004). 
[41] A. E. Nikolaenko, F. D. Angelis, S. A. Boden, N. Papasimakis, P. Ashburn, E. D. Fabrizio, and N. I. Zheludev, Phys. Rev. Lett. 104, 153902 (2010).

[42] A. R. Katko, S. Gu, J. P. Barrett, B. I. Popa, G. Shvets, and S. A. Cummer, Phys. Rev. Lett. 105, 123905 (2010).

[43] E. Kim, F. Wang, W. Wu, Z. N. Yu, and Y. R. Shen, Phys. Rev. B 78, 113102 (2008).

[44] P. Tassin, L. Zhang, Th. Koschny, E. N. Economou, and C. M. Soukoulis, Phys. Rev. Lett. 102, 053901 (2009).

[45] K. F. MacDonald, Z. L. Samson, M. I. Stockman, and N. I. Zheludev, Nature Photon. 3, $55(2009)$.

[46] K. Aydin, K. Guven, M. Kafesaki, L. Zhang, C. M. Soukoulis, and E. Ozbay, Opt. Lett. 29, 2623 (2004).

[47] A. Pinchuk, U. Kreibig, and A. Hilger, Surf. Sci. 557, 269 (2004).

[48] V. P. Drachev, U. K. Chettiar, A. V. Kildishev, H. K. Yuan HK, W. S. Cai, V. M. Shalaev, Opt. Express 16, 1186 (2008).

[49] V. A. Podolskiy and E. E. Narimanov, Opt. Lett. 30, 75 (2005).

[50] D. R. Smith, D. Schurig, M. Rosenbluth, and S. Schultz, Appl. Phys. Lett. 82, 1506 (2003).

[51] D. R. Smith and D. Schurig, Phys. Rev. Lett. 90, 077405 (2003).

[52] D. R. Smith, D. Schurig, J. J. Mock, P. Kolinko, and P. Rye, Appl. Phys. Lett. 84, 2244 (2004).

[53] A. J. Hoffman, L. Alekseyev, S. S. Howard, K. J. Franz, D. Wasserman, V. A. Podolskiy, E. E. Narimanov, D. L. Sivco, and C. Gmachl, Nature Mater. 6, 946 (2007). 
[54] M. G. Silveirinha, P. A. Belov, and C. R. Simovski, Phys. Rev. B 75, 035108 (2007); P. Ikonen, C. Simovski, S. Tretyakov, P. Belov, and Y. Hao, Appl. Phys. Lett. 91, 104102 (2007), and references therein.

[55] R. Wangberg, J. Elser, E. E. Narimanov, and V. A. Podolskiy, J. Opt. Soc. Am. B 23, $498(2006)$.

[56] J. Yao, Z. W. Liu, Y. M. Liu, Y. Wang, C. Sun, G. Bartal, A. M. Stacy, and X. Zhang, Science 321, 930 (2008).

[57] I. V. Lindell, S. A. Tretyakov, K. I. Nikoskinen, and S. Ilvonen, Microwave Opt. Technol. Lett. 31, 129 (2001).

[58] M. Scalora, G. D'Aguanno, N. Mattiucci, M. J. Bloemer, D. de Ceglia, M. Centini, A. Mandatori, C. Sibilia, N. Akozbek, M. G. Cappeddu, M. Fowler, and J. W. Haus, Opt. Express 15, 508 (2007).

[59] B. Wood, J. B. Pendry, and D. P. Tsai, Phys. Rev. B 74, 115116 (2006).

[60] Z. Jacob, L. V. Alekseyev, and E. Narimanov, Opt. Express 14, 8247 (2006).

[61] Y. Liu, G. Bartal, and X. Zhang, Opt. Express 16, 15439 (2008).

[62] A. Fang, Th. Koschny, and C. M. Soukoulis, Phys. Rev. B 79, 245127 (2009).

[63] D. R. Smith, P. Kolinko, and D. Schurig, J. Opt. Soc. Am. B, 21, 1032 (2004).

[64] J. Zhou, Th. Koschny, and C. M. Soukoulis, Opt. Express 16, 11147 (2008).

[65] M. Gorkunov, M. Lapine, E. Shamonina, and K. H. Ringhofer, Eur. Phys. J. B 28, 263 (2002).

[66] J. Zhou, T. Koschny, M. Kafesaki, and C. M. Soukoulis, Phys. Rev. B 80, 035109 (2009).

[67] D. O. Guney, Th. Koschny, and C. M. Soukoulis, Phys. Rev. B 80, 125129 (2009).

[68] S. A. Ramakrishna, and J. B. Pendry, Phys. Rev. B 67, 201101 (2003). 
[69] E. Dulkeith, A. C. Morteani, T. Niedereichholz, T. A. Klar, J. Feldmann, S. A. Levi, F. C. J. M. Van Veggel, D. N. Reinhoudt, M. Moller, and D. I. Gittins, Phys. Rev. Lett. 89, $203002(2002)$.

[70] H. Imahori, Y. Kashiwagi, Y. Endo, T. Hanada, Y. Nishimura, I. Yamazaki, Y. Araki, O. Ito, and S. Fukuzumi, Langmuir 20, 73 (2004).

[71] J. Stehr, J. Crewett, F. Schindler, R. Sperling, G. Von Plessen, U. Lemmer, J. M. Lupton, T. A. Klar, J. Feldmann, A. W. Holleitner, M. Forster, and U. Scherf, Adv. Mater. 15, 1726 (2003).

[72] M. A. Noginov, G. Zhu, M. Bahoura, J. Adegoke, C. E. Small, B. A. Ritzo, V. P. Drachev, and V. M. Shalaev, Opt. Lett. 31, 3022 (2006).

[73] D. J. Bergman and M. I. Stockman, Phys. Rev. Lett. 90, 027402 (2003).

[74] T. A. Klar, A. V. Kildishev, V. P. Drachev, and V. M. Shalaev, IEEE J. Sel. Top. Quantum Electron. 12, 1106 (2006).

[75] A. K. Sarychev and G. Tartakovsky, Phys. Rev. B 75, 085436 (2007).

[76] Y. Sivan, S. Xiao, U. K. Chettiar, A. V. Kildishev, and V. M. Shalaev, Opt. Express 17, 24060 (2009).

[77] Z. G. Dong, H. Liu, T. Li, Z.-H. Zhu, S.-M. Wang, J.-X. Cao, S.-N. Zhu, and X. Zhang, Phys. Rev. B 80, 235116 (2009).

[78] Z. G. Dong, H. Liu, T. Li, Z.-H. Zhu, S.-M. Wang, J.-X. Cao, S.-N. Zhu, and X. Zhang, Appl. Phys. Lett. 96, 044104 (2010).

[79] A. D. Boardman, Yu. G. Rapoport, N. King, and V. N. Malnev, J. Opt. Soc. Am. B 24, A53 (2007).

[80] M. Wegener, J. L. García-Pomar, C. M. Soukoulis, N. Meinzer, M. Ruther, and S. Linden, Opt. Express 16, 19785 (2008). 
[81] A. Fang, T. Koschny, M. Wegener, and C. M. Soukoulis, Phys. Rev. B 79, 241104(R) (2009).

[82] A. Fang, Th. Koschny, and C. M. Soukoulis, J. Opt. 12, 024013 (2010).

[83] A. Fang, Th. Koschny, and C. M. Soukoulis, Phys. Rev. B 82, 121102(R) (2010).

[84] S. Xiao, V. P. Drachev, A. V. Kildishev, X. Ni, U. K. Chettiar, H.-K. Yuan, and V. M. Shalaev, Nature 466, 735 (2010).

[85] E. Plum, V. A. Fedotov, P. Kuo, D. P. Tsai, and N. I. Zheludev, Opt. Express 17, 8548 (2009).

[86] K. S. Yee, IEEE Trans. Antennas Propag. 14, 302 (1966).

[87] A. Taflove, Computational Electrodynamics: The Finite Difference Time Domain Method (Artech House, London, 1995).

[88] A. Taflov, Advances in Computational Electrodynamics: The Finite-Difference TimeDomain Method (Artech House, London, 1998).

[89] A. E. Siegman, Lasers (Hill Valley, California, 1986). See Chaps. 2, 3, 6, and 13.

[90] X. Jiang and C. M. Soukoulis, Phys. Rev. Lett. 85, 70 (2000).

[91] P. Bermel, E. Lidorikis, Y. Fink, and J. D. Joannopoulos, Phys. Rev. B 73, 165125 (2006).

[92] G. Mur, IEEE Trans. Electromagn. Compat. 23, 377 (1981).

[93] Z. P. Liao, H. L. Wong, B. P. Yang, and Y. F. Yuan, Scientia Sinica (series A) XXVII, $1063(1984)$

[94] J. P. Berenger, J. Comput. Phys. 114, 185 (1994).

[95] J. P. Berenger, IEEE Trans. Antennas Propag. 51, 110 (1996).

[96] J. P. Berenger, IEEE Trans. Antennas Propag. 45, 466 (1997). 
[97] Z. Wu, and J. Fang, IEEE Microwave Guided Wave Lett. 6, 335 (1996).

[98] S. D. Gedney, IEEE Trans. Antennas Propag. 44, 1630 (1996).

[99] J. P. Berenger, IEEE AP-S Symp. Dig., 1888 (1997).

[100] J. P. Berenger, IEEE Microwave Guided Wave Lett. 8, 188 (1998).

[101] J. P. Berenger, IEEE Trans. Antennas Propag. 47, 1497 (1999).

[102] J. A. Roden, and S. D. Gedney, Microwave Opt. Technol. Lett. 7, 599 (1994).

[103] A. Ditkowski, K. Dridi, and J. S. Hesthaven, J. Comput. Phys. 170, 39 (2001).

[104] B. Gustafsson, Math. Comp. 29, 396 (1975).

[105] G. Mur, IEEE T. Electromagn. C. 23, 377 (1981).

[106] K. R. Umashankar, IEEE T. Electromagn. C. 24, 397 (1982).

[107] M. Notomi, Phys. Rev. B 62, 10696 (2000).

[108] C. Y. Luo, S. G. Johnson, J. D. Joannopoulos, and J. B. Pendry, Opt. Express 11, 746 (2003).

[109] E. Cubukcu, K. Aydin, E. Ozbay, S. Foteinopoulou, and C. M. Soukoulis, Nature 423, $604(2003)$.

[110] S. Foteinopoulou and C. M. Soukoulis, Phys. Rev. B 67, 235107 (2003).

[111] S. Foteinopoulou and C. M. Soukoulis, Phys. Rev. B 72, 165112 (2005).

[112] R. Moussa, S. Foteinopoulou, L. Zhang, G. Tuttle, K. Guven, E. Ozbay, and C. M. Soukoulis, Phys. Rev. B 71, 085106 (2005).

[113] A. Berrier, M. Mulot, M. Swillo, M. Qiu, L. Thylen, A. Talneau, and S. Anand, Phys. Rev. Lett. 93, 073902 (2004). 
[114] E. Schonbrun, Q. Wu, W. Park, T. Yamashita, C. J. Summers, M. Abashin, and Y. Fainman, Appl. Phys. Lett. 90, 041113 (2007).

[115] X. H. Hu and C. T. Chan, Appl. Phys. Lett. 85, 1520 (2004).

[116] E. D. Palik and G. Ghosh, The electronic handbook of optical constants of solids (Academic, New York, 1999).

[117] J. Elser, R. Wangberg, V. A. Podolskiy, and E. E. Narimanov, Appl. Phys. Lett. 89, $261102(2006)$.

[118] A. Sihvola, Electromagnetic Mixing Formulas and Applications (Institution of Electrical Engineers, London, 1999).

[119] C. A. Foss, G. L. Hornyak, J. A. Stockert, and C. R. Martin, J. Phys. Chem. 98, 2963 (1994).

[120] N. I. Landy, S. Sajuyigbe, J. J. Mock, D. R. Smith, and W. J. Padilla, Phys. Rev. Lett 100, $207402(2008)$.

[121] X. Liu, T. Starr, A. F. Starr, and W. J. Padilla, Phys. Rev. Lett. 104, 207403 (2010).

[122] B. N. Wang, T. Koschny, and C. M. Soukoulis, Phys. Rev. B 80, 033108 (2009).

[123] N. M. Lawandy, Appl. Phys. Lett. 85, 5040 (2004).

[124] A. A. Govyadinov, V. A. Podolskiy, and M. A. Noginov, Appl. Phys. Lett. 91, 191103 (2007).

[125] J. A. Gordon, and R. W. Ziolkowski, Opt. Express 16, 6692 (2008).

[126] D. R. Smith, S. Schultz, P. Markô̂, and C. M. Soukoulis, Phys. Rev. B 65, 195104 (2002).

[127] N. I. Zheludev, S. L. Prosvirnin, N. Papasimakis, and V. A. Fedotov, Nature Photon. 2, 351 (2008). 
[128] J. Zhou, T. Koschny, M. Kafesaki, and C. M. Soukoulis, Phys. Rev. B 80, 035109 (2009).

[129] M. I. Stockman, Phys. Rev. Lett. 98, 177404 (2007).

[130] M. I. Stockman, Phys. Rev. Lett. 99, 189702 (2007).

[131] T. G. Mackay and A. Lakhtakia, Phys. Rev. Lett. 99, 189701 (2007).

[132] P. Kinsler and M. W. McCall, Phys. Rev. Lett. 101, 167401 (2008).

[133] J. Skaar, Phys. Rev. E 73, 026605 (2006).

[134] Th. Koschny, P. Markoŝ, E. N. Economou, D. R. Smith, D. C. Vier, and C. M. Soukoulis, Phys. Rev. B 71, 245105 (2005).

[135] N. Katsarakis, T. Koschny, M. Kafesaki, E. N. Economou, and C. M. Soukoulis, Appl. Phys. Lett. 84, 2943 (2004).

[136] D. Schurig, J. J. Mock, and D. R. Smith, Appl. Phys. Lett. 88, 041109 (2006). 